One-bond, two-bond, and three-bond mechanisms in thermal deazetizations of 2,3-diazabicyclo[2.2.2] oct-2-enes, transazomethane, and 2,3-diazabicyclo[2.2.1] hept-2-ene

Kelli S. Khuong and K. N. Houk*

Supporting Information

\title{
Table of Contents
}

p. $\mathrm{S} 2 \mathrm{DBO}, 2$

p. S23 exo-cyclopropane DBO, 3

p. S30 endo-cyclobutane DBO, 4

p. S38 endo-cyclopropane-exo-cyclobutane, $\mathbf{5}$

p. S47 exo-cyclobutane, 6

p. S58 endo-cyclobutane, 7

p. S69 trans-azomethane, 8

p. $\mathrm{S} 80 \mathrm{DBH}, \mathbf{9}$

p. $\mathrm{S} 94 \mathrm{~N}_{2}$

p. S96 Raw data from scans in Figures 5, 15, 16, 19, 21.

Note: All thermodynamic parameters listed below correspond to 1 atmosphere and $298 \mathrm{~K}$. 


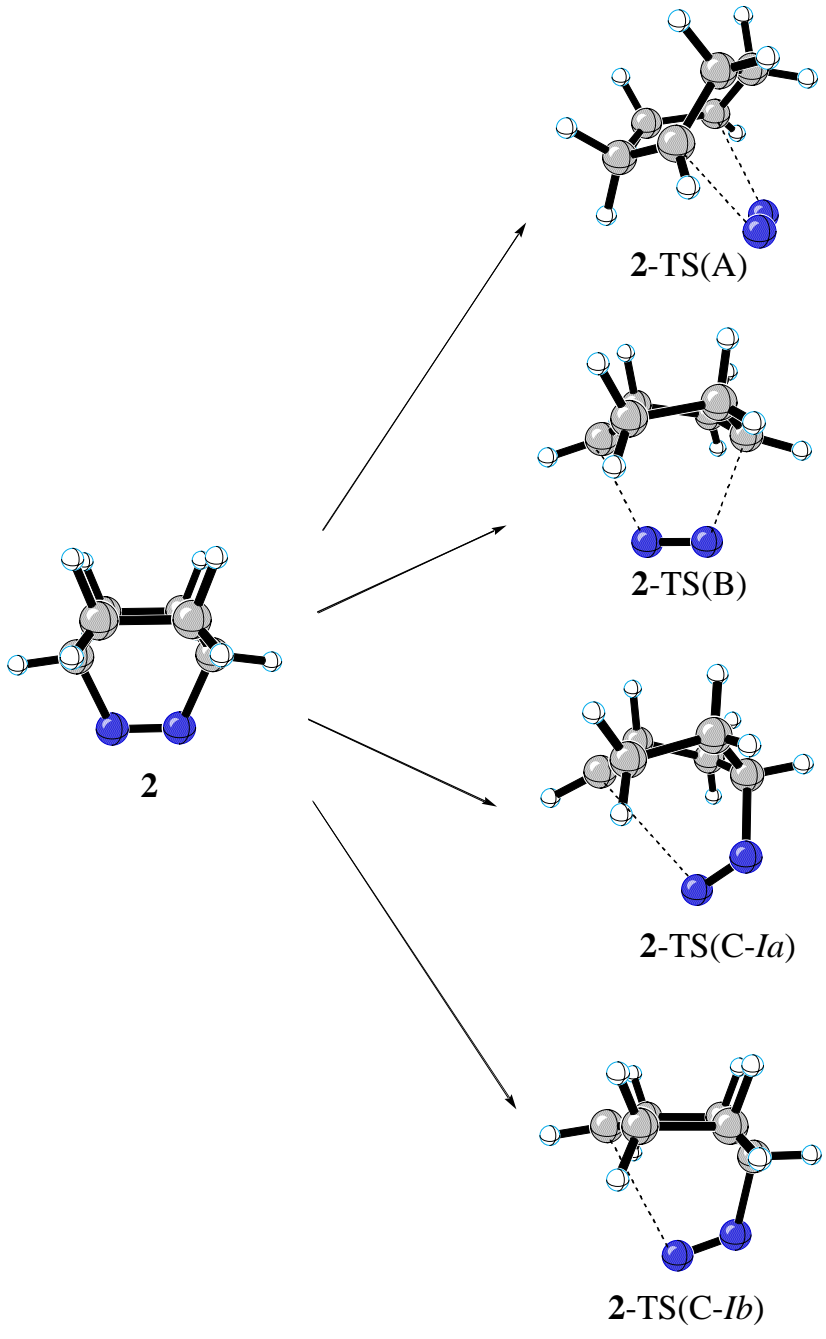

Reactant 2: DBO parent

RB3LYP/6-31G(d)

$E(R B+H F-L Y P)=-344.114798586$

Sum of electronic and zero-point Energies= $\quad-343.955127$

Sum of electronic and thermal Energies=

$-343.948985$

Sum of electronic and thermal Enthalpies=

$-343.948041$

Sum of electronic and thermal Free Energies=

$-343.985143$

TOTAL

E (Thermal) $\mathrm{KCAL} / \mathrm{MOL}$

CV

$S$ 104.049
CAL/MOL-KELVIN CAL/MOL-KELVIN 24.920
78.088

$\begin{array}{ll}1 & 6 \\ 2 & 6 \\ 3 & 1 \\ 4 & 6 \\ 5 & 1 \\ 6 & 1 \\ 7 & 1\end{array}$

-.002591
1.264561
-1.257850
1.268238
2.151346
1.263908
1.257058

$-1.250548$

$-.776569$

1.185346

.771863

$-1.175134$

1.180975

$-1.184242$

$$
\begin{array}{r}
.110574 \\
-.633997 \\
-1.650233 \\
-.632964 \\
-.131387 \\
-1.649741 \\
-1.651394
\end{array}
$$




$\begin{array}{rrrrr}8 & 6 & .002603 & 1.250578 & .111233 \\ 9 & 1 & 2.156382 & 1.165513 & -.128809 \\ 10 & 7 & .001462 & .625176 & 1.463462 \\ 11 & 6 & -1.268317 & -.771390 & -.633254 \\ 12 & 1 & .004666 & 2.333641 & .253151 \\ 13 & 7 & -.001061 & -.626381 & 1.463083 \\ 14 & 1 & -.004635 & -2.333715 & .251677 \\ 15 & 6 & -1.264830 & .777053 & -.633135 \\ 16 & 1 & -1.263838 & -1.179607 & -1.650449 \\ 17 & 1 & -2.156383 & -1.165569 & -.129450 \\ 18 & 1 & -2.151446 & 1.175309 & -.129924\end{array}$

CASPT2 $(10,10) / 6-31 G^{*} / / B 3 L Y P / 6-31 G$ *

Reference energy: $\quad-341.9935239119$

Total energy: $\quad-342.9867256953$

CASPT2 $(6,6) / 6-31 G * / / B 3 L Y P / 6-31 G$ *

Reference energy: $\quad-341.9612782165$

Total energy: $\quad-342.9858320245$

$\begin{array}{ll}\operatorname{CASSCF}(4,4) / 6-31 \mathrm{G}(\mathrm{d}) & \\ \mathrm{E}=-341.9182250496 & -341.747394 \\ \text { Sum of electronic and zero-point Energies= } & -341.741608 \\ \text { Sum of electronic and thermal Energies= } & -341.740663 \\ \text { Sum of electronic and thermal Enthalpies= } & -341.776541 \\ \text { Sum of electronic and thermal Free Energies= } & \end{array}$

E (Thermal) $\mathrm{KCAL} / \mathrm{MOL}$

TOTAL

$\begin{array}{rl}1 & 6 \\ 2 & 6 \\ 3 & 1 \\ 4 & 6 \\ 5 & 1 \\ 6 & 1 \\ 7 & 1 \\ 8 & 6 \\ 9 & 1 \\ 10 & 7 \\ 11 & 6 \\ 12 & 1 \\ 13 & 7 \\ 14 & 1 \\ 15 & 6 \\ 16 & 1 \\ 17 & 1 \\ 18 & 1\end{array}$

110.829
CV CAL/MOL-KELVIN CAL/MOL-KELVIN 22.868

\section{S}

75.510

$\begin{array}{rrr}.00000 \odot & 1.244459 & .099172 \\ -1.261818 & .772274 & -.632569 \\ 1.266261 & -1.174138 & -1.640481 \\ -1.261818 & -.772274 & -.632569 \\ -2.139775 & 1.161193 & -.129980 \\ -1.266261 & -1.174138 & -1.640481 \\ -1.266261 & 1.174138 & -1.640481 \\ .0 \odot \odot \odot \odot \odot & -1.244459 & .099172 \\ -2.139775 & -1.161193 & -.129980 \\ .000 \odot \odot \odot & -.608763 & 1.469224 \\ 1.261818 & .772274 & -.632569 \\ .000 \odot \odot \odot & -2.313929 & .252147 \\ .00000 \odot & .608763 & 1.469224 \\ .0 \odot \odot \odot \odot \odot & 2.313929 & .252147 \\ 1.261818 & -.772274 & -.632569 \\ 1.266261 & 1.174138 & -1.640481 \\ 2.139775 & 1.161193 & -.129980 \\ 2.139775 & -1.161193 & -.129980\end{array}$

$\operatorname{CASPT2}(4,4) / 6-31 G(d) / / \operatorname{CAS}(4,4) / 6-31 G(d)$

Reference energy:

Total energy:
$-341.9164502334$

$-342.9836078369$ 


\begin{tabular}{|c|c|c|c|c|c|c|}
\hline \multicolumn{7}{|c|}{$\operatorname{CASSCF}(6,6) / 6-31 G^{*}$} \\
\hline $\mathrm{E}=$ & -341.9648883 & 542 & & & & \\
\hline Sum 0 & of electronic & and zero-point & nergies= & & -341 & 794709 \\
\hline Sum 0 & of electronic & and thermal Ene & gies= & & -341 & 788866 \\
\hline Sum 0 & of electronic & and thermal Ent & alpies= & & -341 & 787921 \\
\hline Sum 0 & of electronic & and thermal Fre & Energies= & & -341 & 823916 \\
\hline & & E (Thermal) & & & & S \\
\hline & & KCAL/MOL & $\mathrm{CAL} / \mathrm{MOL}$ & ELVIN & & /MOL-KELVIN \\
\hline TOTAL & & 110.456 & & & & 75.757 \\
\hline 1 & 6 & & .000000 & 1.247 & 4493 & . 092945 \\
\hline 2 & 6 & & -1.262402 & .772 & 2727 & -.632139 \\
\hline 3 & 1 & & 1.272639 & -1.173 & 3474 & -1.640494 \\
\hline 4 & 6 & & -1.262402 & -.772 & 2727 & -.632139 \\
\hline 5 & 1 & & -2.139316 & 1.161 & L148 & -.126805 \\
\hline 6 & 1 & & -1.272639 & -1.173 & 3474 & -1.640494 \\
\hline 7 & 1 & & -1.272639 & 1.173 & 3474 & -1.640494 \\
\hline 8 & 6 & & $.00000 \odot$ & -1.247 & 493 & .092945 \\
\hline 9 & 1 & & -2.139316 & -1.161 & L148 & -.126805 \\
\hline 10 & 7 & & .000000 & -.621 & L253 & 1.474456 \\
\hline 11 & 6 & & 1.262402 & .772 & 2727 & -.632139 \\
\hline 12 & 1 & & $.00000 \odot$ & -2.317 & 662 & .241397 \\
\hline 13 & 7 & & $.00000 \odot$ & .621 & L253 & 1.474456 \\
\hline 14 & 1 & & . ๑૦७०७९ & 2.317 & 6662 & . 241397 \\
\hline 15 & 6 & & 1. 262402 & -.772 & 2727 & -.632139 \\
\hline 16 & 1 & & 1.272639 & 1.173 & 3474 & -1.640494 \\
\hline 17 & 1 & & 2.139316 & 1.161 & L148 & - .126805 \\
\hline 18 & 1 & & 2.139316 & -1.161 & 148 & -.126805 \\
\hline
\end{tabular}

$\operatorname{CASPT2}(6,6) / 6-31 G * / / \operatorname{CAS}(6,6) / 6-31 G^{*}$

Reference energy: $\quad-341.963087$

Total energy: $\quad-342.984380$

$\operatorname{CASSCF}(10,8) / 6-31 G^{*}$

$\mathrm{E}=\quad-341.9721176524$

Sum of electronic and zero-point Energies= $\quad-341.802178$

Sum of electronic and thermal Energies= $\quad-341.796312$

Sum of electronic and thermal Enthalpies= $\quad-341.795367$

Sum of electronic and thermal Free Energies $=\quad-341.831403$

$\begin{array}{cr}\text { TOTAL } & 110.325\end{array}$

$\mathrm{E}$ (Thermal) CV S

$\begin{array}{rrrrr}1 & 6 & 0.00000 \odot & 1.251261 & 0.089672 \\ 2 & 6 & -1.262213 & 0.773301 & -0.632414 \\ 3 & 1 & 1.275367 & -1.172319 & -1.641540 \\ 4 & 6 & -1.262213 & -0.773301 & -0.632414 \\ 5 & 1 & -2.139302 & 1.160690 & -0.126399 \\ 6 & 1 & -1.275367 & -1.172319 & -1.641540 \\ 7 & 1 & -1.275367 & 1.172319 & -1.641540 \\ 8 & 6 & 0.00000 \odot & -1.251261 & 0.089672 \\ 9 & 1 & -2.139302 & -1.160690 & -0.126399\end{array}$




$\begin{array}{rrrrr}10 & 7 & 0.000 \odot \odot \odot & -0.618426 & 1.47820 \odot \\ 11 & 6 & 1.262213 & 0.773301 & -0.632414 \\ 12 & 1 & 0.0000 \odot \odot & -2.321299 & 0.239410 \\ 13 & 7 & 0.0000 \odot \odot & 0.618426 & 1.47820 \odot \\ 14 & 1 & 0.00000 \odot & 2.321299 & 0.239410 \\ 15 & 6 & 1.262213 & -0.773301 & -0.632414 \\ 16 & 1 & 1.275367 & 1.172319 & -1.641540 \\ 17 & 1 & 2.139302 & 1.160690 & -0.126399 \\ 18 & 1 & 2.139302 & -1.160690 & -0.126399\end{array}$

CASPT2 $(10,8) / 6-31 G * / / \operatorname{CASSCF}(10,8) / 6-31 G$ *

Reference energy: $\quad-341.9703312783$

Total energy: $\quad-342.9862248444$

\section{Transition structure 2-TS(A): pericyclic loss of nitrogen}

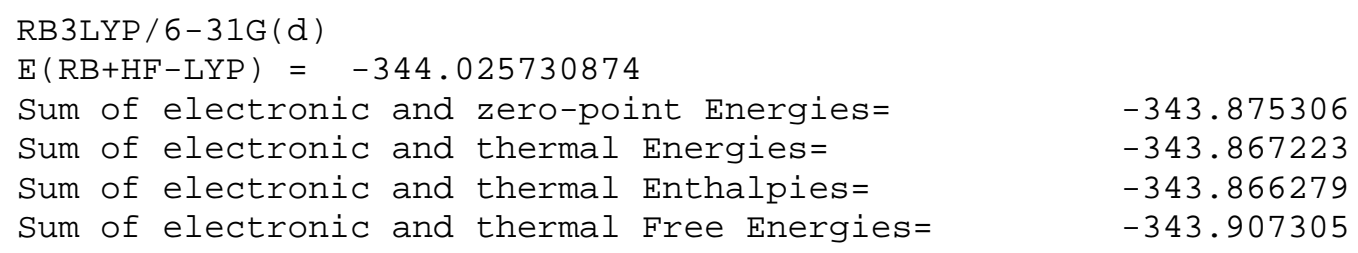

$\begin{array}{rr}\text { TOTAL } & 99.465\end{array}$

$$
\text { E (Thermal) }
$$$$
\text { KCAL/MOL }
$$

$\begin{array}{rr}1 & 6 \\ 2 & 1 \\ 3 & 1 \\ 4 & 6 \\ 5 & 6 \\ 6 & 6 \\ 7 & 7 \\ 8 & 6 \\ 9 & 1 \\ 10 & 1 \\ 11 & 6 \\ 12 & 7 \\ 13 & 1 \\ 14 & 1 \\ 15 & 1 \\ 16 & 1 \\ 17 & 1 \\ 18 & 1\end{array}$

CV CAL/MOL-KELVIN 30.604
$S$ CAL/MOL - KELVIN 86.347
1. 388736 $-2.437632$

$-1.560992$

0.500156

$\odot .924586$

$-0.500146$

○. 261806

$-0.924457$

0.863906

$-0.864168$

$-1.388462$

$-0.262300$

$-1.322552$

$-1.153605$

1.153960

1.323390

1. 560901

2. 437765

CASPT2 $(10,10) / 6-31 G * / / B 3 L Y P / 6-31 G$ *

Reference energy: $\quad-341.9460724105$

Total energy: $\quad-342.9066443536$

Transition structure 2-TS(B): 2-bond CN cleavage

UB3LYP/6-31G(d)

$\mathrm{E}(\mathrm{UB}+\mathrm{HF}-\mathrm{LYP})=-344.041150558$

Sum of electronic and zero-point Energies= $\quad-343.890434$ 
Sum of electronic and thermal Energies=

$-343.882563$

Sum of electronic and thermal Enthalpies=

$-343.881619$

Sum of electronic and thermal Free Energies=

$-343.922521$

E (Thermal) $\mathrm{KCAL} / \mathrm{MOL}$

TOTAL

$\begin{array}{rr}1 & 1 \\ 2 & 6 \\ 3 & 6 \\ 4 & 6 \\ 5 & 1 \\ 6 & 6 \\ 7 & 7 \\ 8 & 1 \\ 9 & 6 \\ 10 & 1 \\ 11 & 7 \\ 12 & 6 \\ 13 & 1 \\ 14 & 1 \\ 15 & 1 \\ 16 & 1 \\ 17 & 1 \\ 18 & 1\end{array}$

CV

CAL/MOL-KELVIN 29.257
$S$

CAL/MOL-KELVIN

86.085

$\begin{array}{rrr}.199330 & -2.301663 & -.054534 \\ -.567122 & -1.523397 & -.158221 \\ -.171278 & -.566750 & -1.245628 \\ -.806987 & -. .789267 & 1.212017 \\ -1.494791 & -2.048772 & -.434790 \\ -.168886 & .567086 & 1.245887 \\ 1.822679 & .178391 & .541892 \\ .148763 & .976876 & 2.198926 \\ -.564150 & 1.524348 & .158739 \\ .144685 & -.976940 & -2.199050 \\ 1.821713 & -.181197 & -.544139 \\ -.807651 & .790382 & -1.210908 \\ -1.490156 & 2.052024 & .436463 \\ .203961 & 2.300808 & .053694 \\ -.456381 & -1.409917 & 2.043072 \\ -1.895284 & -.674245 & 1.354761 \\ -1.896396 & .676553 & -1.351362 \\ -.458033 & 1.410500 & -2.042768\end{array}$

CASPT2 $(10,10) / 6-31 G * / / U B 3 L Y P / 6-31 G *$

Reference energy: $\quad-341.9398360695$

Total energy: $\quad-342.9123857070$

CASPT2 $(6,6) / 6-31 G * / / U B 3 L Y P / 6-31 G *$

Reference energy: $\quad-341.9065255444$

Total energy: $\quad-342.9111734876$

$\operatorname{CASSCF}(4,4) / 6-31 G^{*}$

$\mathrm{E}=\quad-341.8512670520$

Sum of electronic and zero-point Energies= $\quad-341.689568$

Sum of electronic and thermal Energies= $\quad-341.682210$

Sum of electronic and thermal Enthalpies $=\quad-341.681266$

Sum of electronic and thermal Free Energies= $\quad-341.720672$ $\begin{array}{cc} & \text { KCAL/MOL } \\ \text { TOTAL } & 106.085\end{array}$

CV $S$

E (Thermal)
CAL/MOL-KELVIN 26.758

CAL/MOL - KELVIN 82.936

$\begin{array}{ll}1 & 6 \\ 2 & 6 \\ 3 & 7 \\ 4 & 7 \\ 5 & 6 \\ 6 & 6 \\ 7 & 6 \\ 8 & 6\end{array}$

\begin{abstract}
1.205989
$-1.205989$

0.523875

$-0.523875$

0.111876

$-1.238045$

1.238045

$-0.111876$
\end{abstract}
0.595419
$-0.126799$
$-0.595419$
0.214133
$-0.214133$
1.519796
0.760414
$-0.760414$
$-1.519796$ 


$\begin{array}{rrrrr}9 & 1 & 2.150834 & 1.049599 & 0.114501 \\ 10 & 1 & -2.150834 & -1.049599 & 0.114501 \\ 11 & 1 & 0.00000 \odot & 2.335884 & 0.107311 \\ 12 & 1 & -2.060574 & 1.351130 & -0.396439 \\ 13 & 1 & 0.397421 & 1.973500 & -1.546119 \\ 14 & 1 & -1.430812 & 0.627965 & -1.847709 \\ 15 & 1 & 2.060574 & -1.351130 & -0.396439 \\ 16 & 1 & 0.0000 \odot \odot & -2.335884 & 0.107311 \\ 17 & 1 & 1.430812 & -0.627965 & -1.847709 \\ 18 & 1 & -0.397421 & -1.973500 & -1.546119\end{array}$
$\operatorname{CASPT2}(4,4) / 6-31 G * / / \operatorname{CAS}(4,4) / 6-31 G *$
Reference energy: $\quad-341.8495471776$
Total energy: $\quad-342.9141429617$

$\operatorname{CASSCF}(6,6) / 6-31 \mathrm{G}^{*}$
$\mathrm{E}=-341.9056167973$
Sum of electronic and zero-poin
Sum of electronic and thermal En
Sum of electronic and thermal Ent
Sum of electronic and thermal Fre

E (Thermal)
KCAL/MOL
TOTAL
105.995

$\begin{array}{rl}1 & 6 \\ 2 & 6 \\ 3 & 7 \\ 4 & 7 \\ 5 & 6 \\ 6 & 6 \\ 7 & 6 \\ 8 & 6 \\ 9 & 1 \\ 10 & 1 \\ 11 & 1 \\ 12 & 1 \\ 13 & 1 \\ 14 & 1 \\ 15 & 1 \\ 16 & 1 \\ 17 & 1 \\ 18 & 1\end{array}$

$$
\begin{aligned}
& 6 \\
& 6 \\
& 7 \\
& 7 \\
& 6 \\
& 6 \\
& 6 \\
& 6 \\
& 1 \\
& 1 \\
& 1 \\
& 1 \\
& 1 \\
& 1 \\
& 1 \\
& 1 \\
& 1 \\
& 1
\end{aligned}
$$$$
\begin{aligned}
& 6 \\
& 6 \\
& 7 \\
& 7 \\
& 6 \\
& 6 \\
& 6 \\
& 6 \\
& 1 \\
& 1 \\
& 1 \\
& 1 \\
& 1 \\
& 1 \\
& 1 \\
& 1 \\
& 1 \\
& 1
\end{aligned}
$$$$
\begin{aligned}
& 6 \\
& 6 \\
& 6 \\
& 6 \\
& 1.00 \\
& 1.950 \\
& 1 \\
& 1 \\
& 1
\end{aligned}
$$$$
\begin{aligned}
& 6 \\
& 6 \\
& 6 \\
& 6 \\
& 1.00 \\
& 1.950 \\
& 1 \\
& 1 \\
& 1
\end{aligned}
$$$$
\begin{aligned}
& 6 \\
& 6 \\
& 7 \\
& 6 \\
& 6 \\
& 6 \\
& 1 \\
& 1 \\
& 1 \\
& 1 \\
& 1 \\
& 1 \\
& 1
\end{aligned}
$$$$
\begin{aligned}
& 6 \\
& 6 \\
& 7 \\
& 7 \\
& 6 \\
& 6 \\
& 6 \\
& 6 \\
& 1 \\
& 1 \\
& 1 \\
& 1 \\
& 1 \\
& 1 \\
& 1 \\
& 1 \\
& 1 \\
& 1
\end{aligned}
$$

CV

CAL/MOL-KELVIN CAL/MOL-KELVIN 26.588
-341.743976
-341.736703
-341.735758
-341.775591$$
\text { 19. }
$$$$
\text { (1) }
$$$$
\text { 13 }
$$

$$
\begin{aligned}
& \text { KCAL/MOL } \\
& 105.995
\end{aligned}
$$$$
\text { (1) }
$$

Energies=

ergies=

Free Energies=

\section{$S$}

L - KELVIN
83.836
$\operatorname{CASPT2}(6,6) / 6-31 G * / / \operatorname{CAS}(6,6) / 6-31 G^{*}$

$\begin{array}{ll}\text { Reference energy: } & -341.903863 \\ \text { Total energy: } & -342.913604\end{array}$

$\begin{array}{rrr}0.114997 & 0.476045 & 1.256209 \\ 0.117207 & -0.476006 & -1.256093 \\ -1.782805 & 0.167764 & 0.549149 \\ -1.781960 & -0.168101 & -0.551892 \\ 0.597033 & 1.500594 & 0.259616 \\ 0.775681 & 0.876083 & -1.159444 \\ 0.773783 & -0.875972 & 1.160695 \\ 0.597841 & -1.50 \odot 46 \odot & -0.258728 \\ -0.123953 & 0.837997 & 2.240581 \\ -0.120154 & -0.837970 & -2.240841 \\ -0.101974 & 2.330391 & 0.229644 \\ 0.385819 & 1.543869 & -1.919365 \\ 1.547799 & 1.914796 & 0.588286 \\ 1.838958 & 0.762676 & -1.367870 \\ 0.382531 & -1.543804 & 1.919863 \\ -0.100911 & -2.330515 & -0.230028 \\ 1.836650 & -0.762496 & 1.371142 \\ 1.549339 & -1.914293 & -0.585748\end{array}$

\footnotetext{
$\operatorname{CASSCF}(10,8) / 6-31 G^{*}$

$\mathrm{E}=\quad-341.9190637324$

Sum of electronic and zero-point Energies= $\quad-341.757418$

Sum of electronic and thermal Energies= $\quad-341.750112$

Sum of electronic and thermal Enthalpies= $\quad-341.749167$
} 


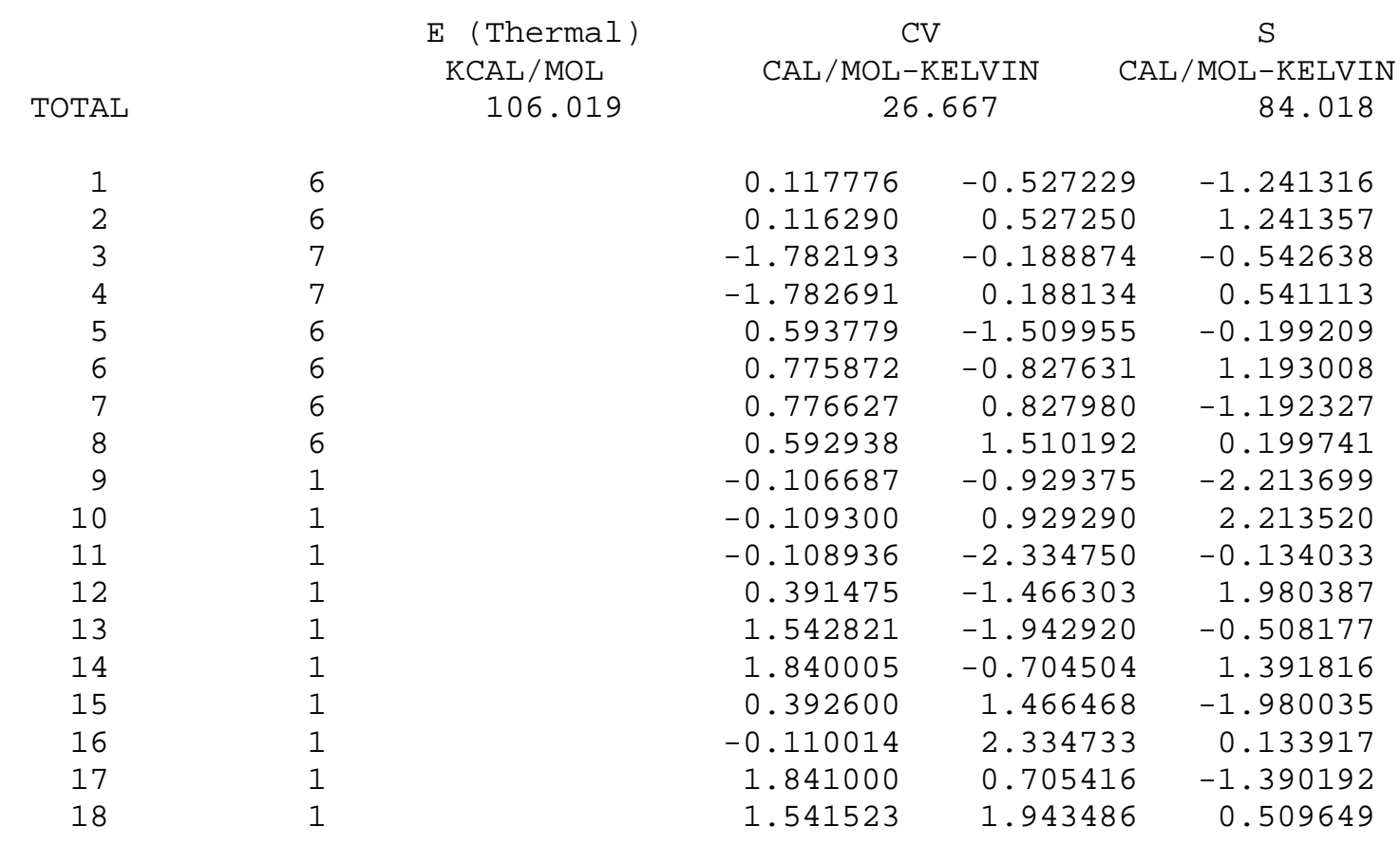

CASPT2 $(10,8) / 6-31 G * / / \operatorname{CAS}(10,8) / 6-31 G$ *

Reference energy: $\quad-341.9173313422$

Total energy: $\quad-342.9170834016$

Transition structure 2-TS(C-Ia): 1-bond CN cleavage to twist-boat geom

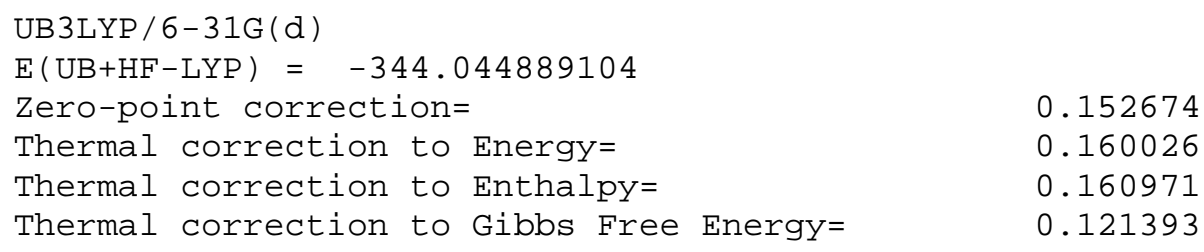

TOTAL

$$
\begin{gathered}
\text { E (Thermal) } \\
\text { KCAL/MOL }
\end{gathered}
$$$$
100.418
$$

CV CAL/MOL-KELVIN CAL/MOL-KELVIN 28.203
$S$ 83.297

$\begin{array}{rl}1 & 6 \\ 2 & 7 \\ 3 & 7 \\ 4 & 6 \\ 5 & 1 \\ 6 & 6 \\ 7 & 1 \\ 8 & 6 \\ 9 & 1 \\ 10 & 6 \\ 11 & 1 \\ 12 & 1 \\ 13 & 6 \\ 14 & 1\end{array}$

-0.782245
-1.798421
-1.551706
1.303553
1.678090
0.816941
0.731766
0.103211
-1.413670
1.426355
0.180516
1.653615
0.036395
-0.614436

0.886452

$-0.235603$

$-1.370502$

$-0.912753$

$-1.926162$

$-0.177883$

1.926099

0.550940

1.755082

$-0.173180$

$-0.836692$

0.108049

1.099514

1.420606 


$\begin{array}{rrrrr}15 & 1 & 0.331440 & -1.959941 & 1.486056 \\ 16 & 1 & -0.478447 & -2.124875 & -0.069165 \\ 17 & 1 & 1.728611 & -1.862954 & -0.844820 \\ 18 & 1 & 2.228143 & -0.977299 & 0.585146\end{array}$
CASPT2 $(10,10) / 6-31 G * / / U B 3 L Y P / 6-31 G$ *
Reference energy: $\quad-341.9274583495$
Total energy: $\quad-342.9151889076$

CASPT2 $(6,6) / 6-31 G^{*} / / U B 3 L Y P / 6-31 G^{*}$

Reference energy: $\quad-341.8954595451$

Total energy: $\quad-342.9140305235$

$\begin{array}{ll}\operatorname{CASSCF}(4,4) / 6-31 \mathrm{G}^{*} & \\ \mathrm{E}=-341.8514809309 & -341.687989 \\ \text { Sum of electronic and zero-point Energies= } & -341.681047 \\ \text { Sum of electronic and thermal Energies= } & -341.680103 \\ \text { Sum of electronic and thermal Enthalpies= } & -341.719097 \\ \text { Sum of electronic and thermal Free Energies= }\end{array}$
CV $S$ $\begin{array}{cr} & \text { KCAL/MOL } \\ \text { TOTAL } & 106.949\end{array}$
E (Thermal)
CAL/MOL-KELVIN CAL/MOL-KELVIN
$\begin{array}{ll}26.137 & 82.071\end{array}$

$\begin{array}{rrrrr}1 & 6 & -0.765241 & -0.131703 & 0.874684 \\ 2 & 7 & -1.832418 & 0.203752 & -0.209653 \\ 3 & 7 & -1.713051 & -0.214125 & -1.283987 \\ 4 & 6 & 1.357500 & 0.143045 & -0.933906 \\ 5 & 1 & 1.965199 & 0.221460 & -1.817480 \\ 6 & 6 & 0.895067 & 1.411284 & -0.267189 \\ 7 & 1 & 0.795315 & 0.932999 & 1.844126 \\ 8 & 6 & 0.054448 & -1.398248 & 0.576620 \\ 9 & 1 & -1.369531 & -0.270753 & 1.760079 \\ 10 & 6 & 1.406808 & -1.112775 & -0.113556 \\ 11 & 1 & 0.276489 & 1.980190 & -0.955581 \\ 12 & 1 & 1.742311 & 2.053320 & -0.026884 \\ 13 & 6 & 0.108585 & 1.113345 & 1.021202 \\ 14 & 1 & -0.500196 & 1.965849 & 1.300998 \\ 15 & 1 & 0.232031 & -1.910933 & 1.516774 \\ 16 & 1 & -0.539892 & -2.067899 & -0.032896 \\ 17 & 1 & 1.689190 & -1.960024 & -0.728242 \\ 18 & 1 & 2.184375 & -1.021285 & 0.647453\end{array}$

CASPT2 $(4,4) / 6-31 G * / / \operatorname{CAS}(4,4) / 6-31 G *$

Reference energy: $\quad-341.8496707295$

Total energy: $\quad-342.9144841569$

Transition structure 2-TS(C-Ib): 1-bond CN cleavage to chair-like geom

UB3LYP/6-31G(d)

$E(U B+H F-L Y P)=-344.043857131$

Sum of electronic and zero-point Energies= -343.891249

Sum of electronic and thermal Energies= $\quad-343.883696$ 
Sum of electronic and thermal Enthalpies= $\quad-343.882752$

Sum of electronic and thermal Free Energies= $\quad-343.923622$

$\begin{array}{cc} & \text { KCAL/MOL } \\ \text { TOTAL } & 100.502\end{array}$

E (Thermal)
CV

CAL/MOL-KELVIN CAL/MOL-KELVIN

28.002

$S$

$\begin{array}{rrrrr}1 & 6 & 1.473365 & 0.000812 & 0.688704 \\ 2 & 6 & 1.056197 & 1.334767 & 0.145861 \\ 3 & 1 & 0.385978 & -1.239817 & -1.931265 \\ 4 & 6 & -0.055403 & 1.260978 & -0.926419 \\ 5 & 1 & 0.713751 & 1.958777 & 0.984253 \\ 6 & 1 & 0.387600 & 1.237753 & -1.931138 \\ 7 & 1 & 1.918137 & 1.881849 & -0.274936 \\ 8 & 6 & -0.917796 & -0.00 \odot 298 & -0.816631 \\ 9 & 1 & -0.685806 & 2.156455 & -0.886156 \\ 10 & 7 & -1.723200 & 0.000508 & 0.497990 \\ 11 & 6 & 1.058616 & -1.333458 & 0.144728 \\ 12 & 1 & -1.684553 & -0.000663 & -1.595099 \\ 13 & 7 & -1.201689 & -0.001896 & 1.557674 \\ 14 & 1 & 2.124688 & 0.001023 & 1.559424 \\ 15 & 6 & -0.055127 & -1.261405 & -0.925682 \\ 16 & 1 & 1.921296 & -1.877755 & -0.278196 \\ 17 & 1 & 0.719293 & -1.959345 & 0.982952 \\ 18 & 1 & -0.685284 & -2.156936 & -0.882849\end{array}$

CASPT2 $(10,10) / 6-31 G * / / U B 3 L Y P / 6-31 G$ *

Reference energy: $\quad-341.9257969881$

Total energy: $\quad-342.9138386540$

CASPT2 $(6,6) / 6-31 G^{*} / / U B 3 L Y P / 6-31 G^{*}$

Reference energy: $\quad-341.8939598917$

Total energy: $\quad-342.9126525054$ 

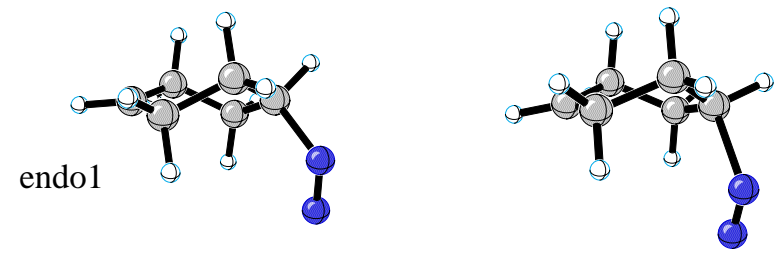
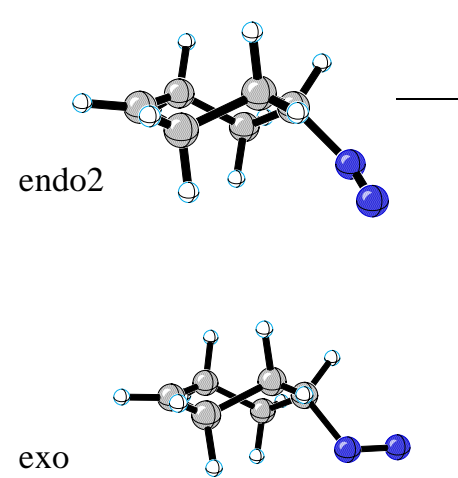

diazenyl diradical (twist boat geom)
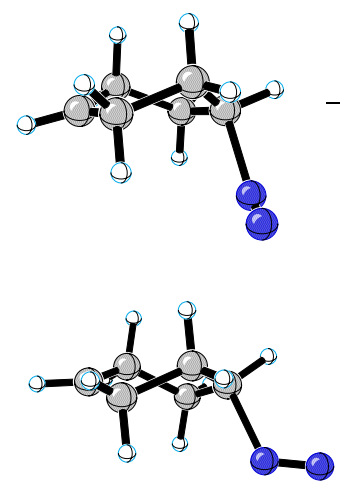

2-TS (C-IIa)

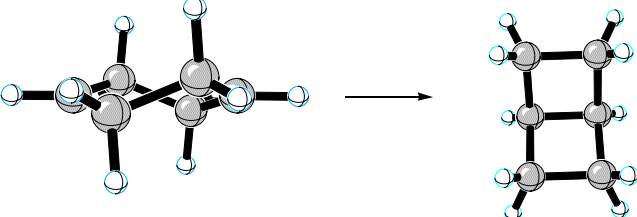

twist boat 1,4-diyl bicyclo[2.2.0]hexane

Intermediate from 2: twist boat diazenyl diradical endo1 $\mathrm{N}=\mathrm{N}$

UB3LYP/6-31G(d)

$\mathrm{E}(\mathrm{UB}+\mathrm{HF}-\mathrm{LYP})=-344.046414502$

Sum of electronic and zero-point Energies= -343.894241

Sum of electronic and thermal Energies=

$-343.885811$

Sum of electronic and thermal Enthalpies=

$-343.884867$

Sum of electronic and thermal Free Energies=

$-343.928242$

TOTAL

$$
\text { E (Thermal) }
$$
$\mathrm{KCAL} / \mathrm{MOL}$ 100.780
CV CAL/MOL-KELVIN 30.532
$S$

CAL/MOL - KELVIN

91.290

$\begin{array}{rl}1 & 6 \\ 2 & 7 \\ 3 & 7 \\ 4 & 6 \\ 5 & 1 \\ 6 & 6 \\ 7 & 1 \\ 8 & 6 \\ 9 & 1 \\ 10 & 6 \\ 11 & 1 \\ 12 & 1 \\ 13 & 6 \\ 14 & 1 \\ 15 & 1 \\ 16 & 1 \\ 17 & 1 \\ 18 & 1\end{array}$

$$
\begin{array}{r}
-0.075202 \\
0.275652 \\
-0.258721 \\
0.078664 \\
0.115494 \\
1.284424 \\
1.218686 \\
-1.304990 \\
-0.270833 \\
-1.180930 \\
1.373111 \\
2.208653 \\
1.207272 \\
2.077579 \\
-2.214295 \\
-1.405927 \\
-2.061523 \\
-1.214884
\end{array}
$$

0.756631

0.048376

$-0.914591$

$-0.559054$

$-1.107066$

$-0.492200$

1.644494

0.133852

1.781173

0.128163

$-1.401586$

$-0.459250$

0.739690

0.798063

0.658421

$-0.901378$

$-0.361729$

1.169868 


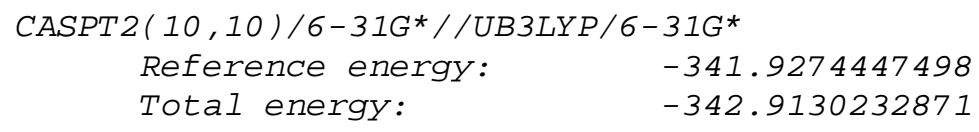

Intermediate from 2: twist boat diazenyl diradical, endo2 $\mathrm{N}=\mathrm{N}$

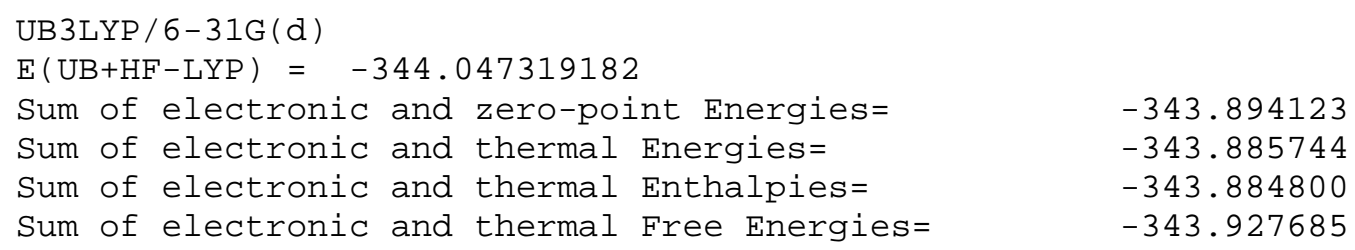

-0.656509
-2.040159
-2.654310
2.040984
3.049411
0.903320
0.282256
0.291157
-0.859960
1.768506
0.423761
1.257605
-0.159238
-1.014865
0.080073
0.088173
2.412966
2.012547

Intermediate from 2: twist boat diazenyl diradical, exo $\mathrm{N}=\mathrm{N}$

UB3LYP/ $6-31 G(d)$

$E(U B+H F-L Y P)=-344.047267245$

Sum of electronic and zero-point Energies= -343.894914

Sum of electronic and thermal Energies= $\quad-343.886562$

Sum of electronic and thermal Enthalpies $=\quad-343.885618$

Sum of electronic and thermal Free Energies= $\quad-343.928434$

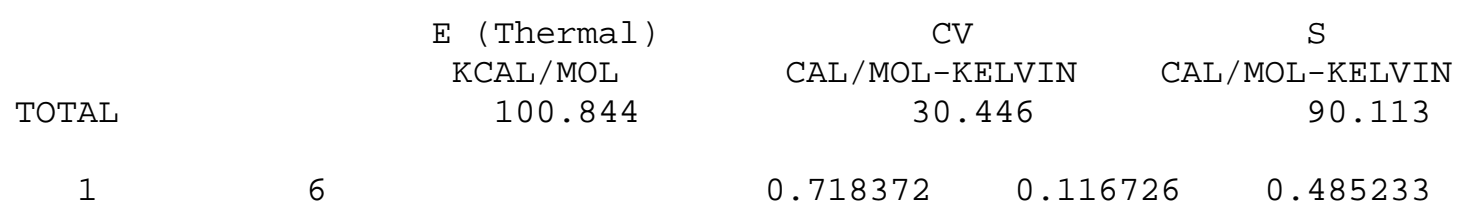




$\begin{array}{rrrrr}2 & 6 & -0.087826 & -1.190797 & 0.615055 \\ 3 & 6 & -1.243447 & -1.287517 & -0.397860 \\ 4 & 1 & 0.590418 & -2.046521 & 0.520552 \\ 5 & 1 & -0.504099 & -1.229076 & 1.629281 \\ 6 & 6 & -2.150727 & -0.102525 & -0.271435 \\ 7 & 1 & -0.818313 & -1.360519 & -1.416301 \\ 8 & 6 & -0.075642 & 1.280888 & -0.137717 \\ 9 & 1 & -3.204020 & -0.183536 & -0.523308 \\ 10 & 1 & 1.145440 & 0.399933 & 1.452871 \\ 11 & 6 & -1.579706 & 1.204006 & 0.188904 \\ 12 & 1 & -1.796158 & -2.223676 & -0.245181 \\ 13 & 1 & 0.352612 & 2.235233 & 0.187343 \\ 14 & 1 & -1.724736 & 1.345528 & 1.276886 \\ 15 & 7 & 1.912982 & -0.153299 & -0.457530 \\ 16 & 7 & 3.019890 & 0.104272 & -0.151925 \\ 17 & 1 & 0.044424 & 1.233048 & -1.227175 \\ 18 & 1 & -2.101811 & 2.048096 & -0.281855\end{array}$

CASPT2 $(10,10) / 6-31 G * / / U B 3 L Y P / 6-31 G$ *

Reference energy: $\quad-341.929755$

Total energy: $\quad-342.914337$

$\operatorname{CASSCF}(4,4) / 6-31 G^{*}$

$\mathrm{E}=-341.8544397844$

Sum of electronic and zero-point Energies=

Sum of electronic and thermal Energies=

Sum of electronic and thermal Enthalpies=

Sum of electronic and thermal Free Energies=

$-341.690872$

$-341.683044$

$-341.682099$

$-341.723672$

E (Thermal) $\mathrm{KCAL} / \mathrm{MOL}$

CV

$S$

TOTAL 107.553

CAL/MOL-KELVIN CAL/MOL-KELVIN 28.184

87.497

$\begin{array}{rrrrr}1 & 6 & -0.729691 & -0.131219 & 0.465700 \\ 2 & 6 & 0.070884 & 1.171209 & 0.630032 \\ 3 & 6 & 1.210423 & 1.296978 & -0.384674 \\ 4 & 1 & -0.603275 & 2.018198 & 0.565090 \\ 5 & 1 & 0.493007 & 1.184652 & 1.630641 \\ 6 & 6 & 2.146565 & 0.126939 & -0.252519 \\ 7 & 1 & 0.797274 & 1.353302 & -1.392836 \\ 8 & 6 & 0.086676 & -1.280082 & -0.148091 \\ 9 & 1 & 3.085721 & 0.143813 & -0.775310 \\ 10 & 1 & -1.150474 & -0.426779 & 1.419258 \\ 11 & 6 & 1.575224 & -1.187854 & 0.213849 \\ 12 & 1 & 1.744406 & 2.229772 & -0.225664 \\ 13 & 1 & -0.331730 & -2.231147 & 0.164212 \\ 14 & 1 & 1.696194 & -1.298055 & 1.292381 \\ 15 & 7 & -1.887979 & 0.146698 & -0.484936 \\ 16 & 7 & -2.967852 & -0.103224 & -0.138221 \\ 17 & 1 & 2.110052 & -2.019713 & -0.233276 \\ 18 & 1 & -0.010846 & -1.234186 & -1.228184\end{array}$

$\operatorname{CASPT2}(4,4) / 6-31 G * / / \operatorname{CAS}(4,4) / 6-31 G *$

Reference energy: $\quad-341.8526344155$

Total energy: $\quad-342.9152347718$ 
Transition structure from 2: TS(C-IIa) cleave 2nd CN, twist boat geom, endo1 $\underline{\mathbf{N}=\mathbf{N}}$

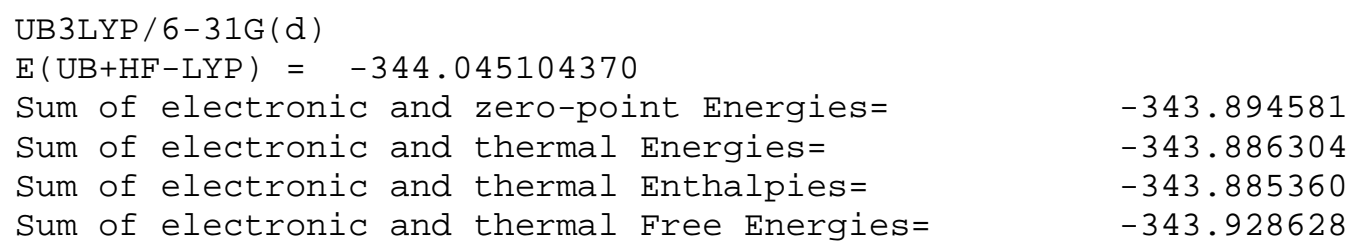

$\begin{array}{rr}\text { KCAL/MOL } \\ \text { TOTAL } & 99.649\end{array}$

$$
\begin{gathered}
\text { E (Thermal) } \\
\text { KCAL/MOL } \\
99.649
\end{gathered}
$$

CV CAL/MOL-KELVIN 29.573
$S$ CAL/MOL-KELVIN 91.065

$\begin{array}{rrrrr}1 & 6 & 0.628168 & 0.088340 & 0.876295 \\ 2 & 6 & -0.215206 & -1.174478 & 0.865546 \\ 3 & 6 & -0.999824 & -1.335173 & -0.447354 \\ 4 & 1 & 0.427012 & -2.043735 & 1.047369 \\ 5 & 1 & -0.920327 & -1.121380 & 1.708088 \\ 6 & 6 & -1.771319 & -0.093823 & -0.774681 \\ 7 & 1 & -0.290637 & -1.579188 & -1.258658 \\ 8 & 6 & -0.034677 & 1.355337 & 0.339920 \\ 9 & 1 & -2.514699 & -0.113851 & -1.566444 \\ 10 & 1 & 1.150474 & 0.224994 & 1.824575 \\ 11 & 6 & -1.535191 & 1.156898 & 0.011115 \\ 12 & 1 & -1.665878 & -2.209354 & -0.379367 \\ 13 & 1 & 0.087607 & 2.174817 & 1.058135 \\ 14 & 1 & -2.102557 & 1.134244 & 0.961062 \\ 15 & 7 & 2.021415 & -0.340977 & -0.099730 \\ 16 & 7 & 2.381176 & 0.319634 & -0.973876 \\ 17 & 1 & -1.909022 & 2.034429 & -0.530185 \\ 18 & 1 & 0.488184 & 1.665815 & -0.574382\end{array}$

CASPT2 $(10,10) / 6-31 G * / / U B 3 L Y P / 6-31 G *$

Reference energy: $\quad-341.9321006264$

Total energy: -342.9102149648

CASPT2 $(6,6) / 6-31 G * / / U B 3 L Y P / 6-31 G *$

Reference energy: $\quad-341.9006786837$

Total energy: $\quad-342.9091270366$

Transition structure from 2: TS(C-IIa) cleave 2nd $\mathrm{CN}$, twist boat geom, exo $\underline{\mathbf{N}=\mathbf{N}}$

UB3LYP/6-31G(d)

$E(U B+H F-L Y P)=-344.044345361$

Sum of electronic and zero-point Energies= -343.894296

Sum of electronic and thermal Energies= -343.885883

Sum of electronic and thermal Enthalpies $=\quad-343.884939$

Sum of electronic and thermal Free Energies= $\quad-343.928231$
E (Thermal)
CV
S 
$\begin{array}{cr} & \mathrm{KCAL} / \mathrm{MOL} \\ \text { TOTAL } & 99.437\end{array}$

$$
\begin{array}{cc}
\text { CAL/MOL-KELVIN } & \text { CAL/MOL-KELVIN } \\
29.933 & 91.118
\end{array}
$$

$\begin{array}{rrrrr}1 & 6 & -0.632281 & -0.224258 & 0.608052 \\ 2 & 6 & 0.069643 & 1.115954 & 0.750156 \\ 3 & 6 & 1.106858 & 1.355806 & -0.360463 \\ 4 & 1 & -0.671206 & 1.924523 & 0.778336 \\ 5 & 1 & 0.579051 & 1.131825 & 1.725153 \\ 6 & 6 & 2.089910 & 0.227145 & -0.426722 \\ 7 & 1 & 0.571996 & 1.471617 & -1.321454 \\ 8 & 6 & 0.177137 & -1.347081 & -0.030216 \\ 9 & 1 & 3.067170 & 0.380192 & -0.875423 \\ 10 & 1 & -1.193772 & -0.511604 & 1.497690 \\ 11 & 6 & 1.702697 & -1.120466 & 0.098687 \\ 12 & 1 & 1.619292 & 2.313738 & -0.196272 \\ 13 & 1 & -0.109480 & -2.314003 & 0.397517 \\ 14 & 1 & 1.992532 & -1.233781 & 1.160453 \\ 15 & 7 & -1.963159 & 0.091518 & -0.555352 \\ 16 & 7 & -3.052605 & -0.076801 & -0.225947 \\ 17 & 1 & 2.238738 & -1.917330 & -0.433719 \\ 18 & 1 & -0.067764 & -1.390799 & -1.100162\end{array}$

CASPT2 $(10,10) / 6-31 G * / / U B 3 L Y P / 6-31 G$ *

Reference energy: $\quad-341.9348287135$

Total energy: $\quad-342.9093194287$

$\operatorname{CASSCF}(4,4) / 6-31 \mathrm{G}^{*}$
$\mathrm{E}=-341.8523081582$
Sum of electronic and zero-point Energies=
Sum of electronic and thermal Energies=
Sum of electronic and thermal Enthalpies=
Sum of electronic and thermal Free Energies=

TOTAL

E (Thermal)

CV S

$\mathrm{KCAL} / \mathrm{MOL}$ 105.911
CAL/MOL-KELVIN CAL/MOL-KELVIN 27.955

88.645

$\begin{array}{rrr}-0.661674 & -0.159537 & \odot .550336 \\ 0.089948 & 1.161823 & 0.675334 \\ 1.184457 & 1.312237 & -0.385433 \\ -0.612049 & 1.987718 & 0.627838 \\ 0.549169 & 1.202262 & 1.660176 \\ 2.146824 & 0.158452 & -0.308137 \\ 0.725751 & 1.367757 & -1.373775 \\ 0.121416 & -1.295742 & -0.101408 \\ 3.067689 & 0.206577 & -0.860672 \\ -1.141510 & -0.444970 & 1.475291 \\ 1.630847 & -1.176570 & 0.164739 \\ 1.709269 & 2.252989 & -0.243971 \\ -0.256245 & -2.253026 & 0.241689 \\ 1.822602 & -1.304948 & 1.230838 \\ -1.986053 & 0.155591 & -0.535628 \\ -3.020747 & -0.122648 & -0.153313\end{array}$




\begin{tabular}{rrrrr}
17 & 1 & 2.153089 & -1.987996 & -0.332778 \\
18 & 1 & -0.041067 & -1.260943 & -1.174633 \\
& & \\
\multicolumn{4}{r}{ CASPT2 (4,4)/6-31G*//CAS $(4,4) / 6-31 G^{*}$} \\
Reference energy: & -341.8505005126 & \\
Total energy: & -342.9106550792 &
\end{tabular}

Transition structure from 2: TS(C-IIb) cleave 2nd CN, twist boat geom, endo2 $\underline{\mathrm{N}=\mathrm{N}}$

\begin{tabular}{|c|c|c|c|c|c|c|}
\hline \multicolumn{7}{|c|}{ UB3LYP/6-31G(d) } \\
\hline$E(U B+H$ & $F-L Y P)=-$ & 344.044576751 & & & & \\
\hline Sum of & electronic & and zero-point & nergies= & & -343 & 894143 \\
\hline Sum of & electronic & and thermal En & gies= & & -343 & 885868 \\
\hline Sum of & electronic & and thermal En & alpies= & & -343 & 884923 \\
\hline Sum of & electronic & and thermal $\mathrm{Fr}$ & Energies= & & -343 & 927828 \\
\hline & & E (Thermal) & & & & $\mathrm{S}$ \\
\hline & & $\mathrm{KCAL} / \mathrm{MOL}$ & $\mathrm{CAL} / \mathrm{MOL}$ & ELVIN & & /MOL - KELVIN \\
\hline TOTAL & & 99.592 & & 623 & & 90.301 \\
\hline 1 & 6 & & -0.540714 & $-0.64 C$ & 171 & 0.672657 \\
\hline 2 & 6 & & -0.186038 & 0.783 & 3874 & 1.053637 \\
\hline 3 & 6 & & 0.543450 & $1.52 \varsigma$ & 9188 & -0.077312 \\
\hline 4 & 1 & & -1.092554 & 1.325 & $5 \odot 99$ & 1.346894 \\
\hline 5 & 1 & & 0.458363 & $0.74 c$ & 966 & 1.944395 \\
\hline 6 & 6 & & 1.655957 & 0.705 & 9680 & -0.652317 \\
\hline 7 & 1 & & -0.188899 & 1.793 & 3286 & $-\odot .859226$ \\
\hline 8 & 6 & & 0.560405 & -1.466 & 5016 & 0.007583 \\
\hline 9 & 1 & & 2.331484 & 1.15 & 5241 & -1.377092 \\
\hline 10 & 1 & & -1.062034 & -1.173 & 3165 & 1.469709 \\
\hline 11 & 6 & & 1.886739 & -0.68 & 1993 & -0.158303 \\
\hline 12 & 1 & & $\odot .915093$ & 2.496 & 178 & 0.299264 \\
\hline 13 & 1 & & $\odot .741175$ & -2.38 & 4060 & 0.580417 \\
\hline 14 & 1 & & $2.4 \odot 2597$ & -0.66 & 4438 & $\odot .820780$ \\
\hline 15 & 7 & & -1.867749 & $-0.58 \varsigma$ & 940 & -0.485232 \\
\hline 16 & 7 & & -2.530409 & $0.34 \varsigma$ & 9562 & -0.584611 \\
\hline 17 & 1 & & 2.555403 & -1.231 & L570 & -0.831047 \\
\hline 18 & 1 & & $\odot .207679$ & -1.785 & 5553 & -0.980874 \\
\hline
\end{tabular}

CASPT2 $(10,10) / 6-31 G * / / U B 3 L Y P / 6-31 G *$

Reference energy: $\quad-341.9321817024$

Total energy: $\quad-342.9101735600$

Intermediate: twist boat 1,4-diyl

UB3LYP/6-31G(d)

$\mathrm{E}(\mathrm{UB}+\mathrm{HF}-\mathrm{LYP})=-234.545662755$

Sum of electronic and zero-point Energies= $\quad-234.405026$

Sum of electronic and thermal Energies= $\quad-234.398336$

Sum of electronic and thermal Enthalpies= $\quad-234.397392$

Sum of electronic and thermal Free Energies= $\quad-234.435223$ 
E (Thermal) $\mathrm{KCAL} / \mathrm{MOL}$ 92.449
CV CAL/MOL-KELVIN CAL/MOL-KELVIN 24.479
2.531031

$-0.558554$

0.651595

$-1.446834$

$-0.770468$

$-0.651618$

1.446833

0.558423

$-1.174731$

$-0.685499$

1.375320

$\odot .770488$

0.685634

1.174731

$-2.531011$

$-1.375321$
-0.109174
-1.368907
-0.283734
-0.040378
$0.31600 \odot$
-0.283677
-0.040413
-1.368967
0.119622
1.414882
-0.007404
0.315937
1.414823
0.119473
-0.109510
-0.007245

$-0.104377$

2.077640

CASPT2 $(6,6) / 6-31 G * / / U B 3 L Y P / 6-31 G$ *

Reference energy: $\quad-232.9679925695$

Total energy: $\quad-233.6963645318$

$\operatorname{CASSCF}(2,2) / 6-31 \mathrm{G} *$

$E=-232.9394443237$

Sum of electronic and zero-point Energies= $\quad-232.788228$

Sum of electronic and thermal Energies= $\quad-232.781984$

Sum of electronic and thermal Enthalpies= $\quad-232.781040$

Sum of electronic and thermal Free Energies= $\quad-232.817534$

TOTAL

E (Thermal) $\mathrm{KCAL} / \mathrm{MOL}$ 98.808
CV

CAL/MOL-KELVIN CAL/MOL-KELVIN 22.444
76.808

$\begin{array}{rl}1 & 1 \\ 2 & 1 \\ 3 & 6 \\ 4 & 6 \\ 5 & 6 \\ 6 & 6 \\ 7 & 6 \\ 8 & 1 \\ 9 & 1 \\ 10 & 1 \\ 11 & 1 \\ 12 & 6 \\ 13 & 1 \\ 14 & 1 \\ 15 & 1 \\ 16 & 1\end{array}$

2.198330
-1.409571
1.252712
-1.252712
-0.071701
-1.252712
1.252712
1.409571
-2.086041
-0.066482
0.149121
0.071701
0.066482
2.086041
-2.198330
-0.149121

1.171023

1.214981

$-0.844424$

$-0.662623$

$-1.410092$

0.844424

0.662623

$-1.214981$

1.224833

$-1.344492$

2.466673

1.410092

1.344492

$-1.224833$

$-1.171023$

$-2.466673$
$-0.285914$

$-1.212035$

$-0.200166$

$-0.211198$

0.340342

$-0.200166$

$-0.211198$

$-1.212035$

0.389332

1.430553

0.104198

0.340342

1.430553

$\odot .389332$

$-0.285914$

$\odot .104198$ 
Product: bicyclo[2.2.0]hexane

RB3LYP/6-31G(d)

$E(R B+H F-L Y P)=-234.598091050$

Sum of electronic and zero-point Energies $=\quad-234.452032$

Sum of electronic and thermal Energies=

$-234.446600$

Sum of electronic and thermal Enthalpies=

$-234.445656$

Sum of electronic and thermal Free Energies=

$-234.480551$

TOTAL

E (Thermal) $\mathrm{KCAL} / \mathrm{MOL}$

CV

$S$

95.062

CAL/MOL-KELVIN CAL/MOL-KELVIN 20.677

73.443

$\begin{array}{rl}1 & 1 \\ 2 & 6 \\ 3 & 6 \\ 4 & 6 \\ 5 & 6 \\ 6 & 1 \\ 7 & 1 \\ 8 & 1 \\ 9 & 6 \\ 10 & 1 \\ 11 & 1 \\ 12 & 1 \\ 13 & 1 \\ 14 & 6 \\ 15 & 1 \\ 16 & 1\end{array}$

-0.892459
-0.505411
-1.499617
0.505411
-0.505411
-0.899434
0.892459
-0.142416
1.499617
0.899434
-1.736579
-2.444083
1.736579
0.505411
2.444083
0.142416

2.313337

$\odot .298172$

1.444429

$-0.244075$

$0.242304 \quad-0.263217$

$\odot .602757$

$-0.602757$

0.579040

$-1.057786$

$\odot .579040$

$-2.313337$

1.492406

0.298172

1.789178

$-0.242304$

$-1.219409$

1.057786

$-0.263217$

$-0.169796$

1.492406

$\odot .451640$

$-1.251093$

0.249439

0.169796

$-1.444429$

$-0.451640$

$-1.251093$

$-0.244075$

0.249439

$-1.789178$

$-1.219409$ 


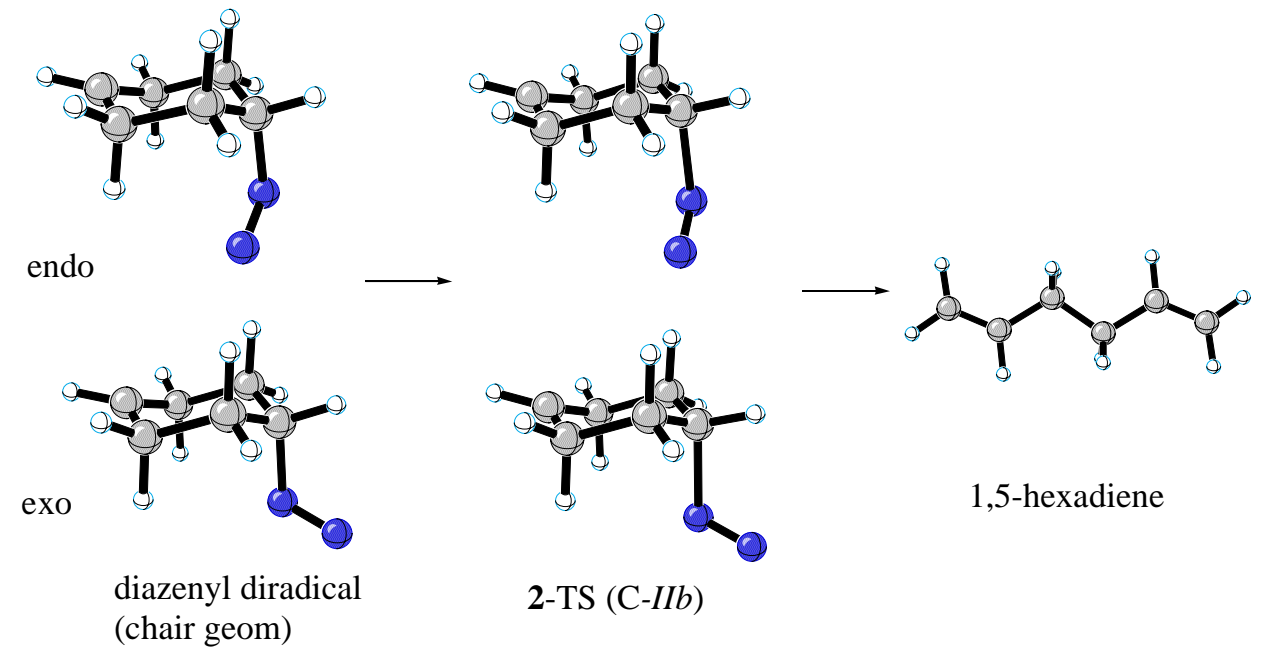

Intermediate from 2: chair-like diazenyl diradical, endo $\mathrm{N}=\mathrm{N}$

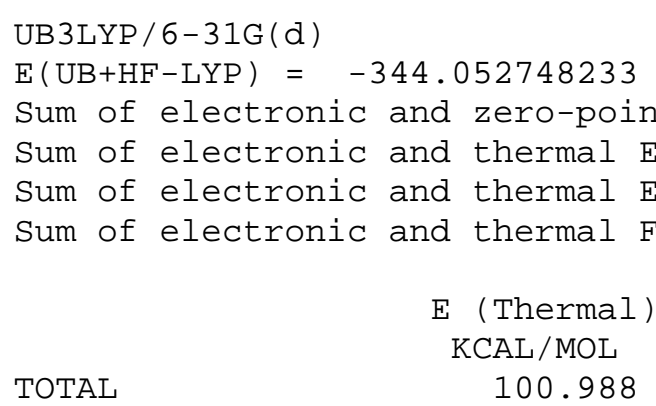

100.988

$\begin{array}{rl}1 & 6 \\ 2 & 6 \\ 3 & 1 \\ 4 & 6 \\ 5 & 1 \\ 6 & 1 \\ 7 & 1 \\ 8 & 6 \\ 9 & 1 \\ 10 & 7 \\ 11 & 6 \\ 12 & 1 \\ 13 & 7 \\ 14 & 1 \\ 15 & 6 \\ 16 & 1 \\ 17 & 1 \\ 18 & 1\end{array}$

-343.899809
-343.891814
-343.890869
-343.932010

CV

CAL/MOL-KELVIN CAL/MOL-KELVIN 30.037

$$
\begin{array}{rrr}
1.768274 & .459189 & -.462258 \\
1.356057 & -.972627 & -.596898 \\
.329013 & 1.028320 & 1.974644 \\
.431374 & -1.419745 & .555028 \\
.808987 & -1.125025 & -1.547163 \\
1.011777 & -1.500122 & 1.482626 \\
2.231578 & -1.630960 & -.648163 \\
-.700466 & -.417779 & .796857 \\
.006708 & -2.408431 & .346096 \\
-1.684065 & -.540530 & -.401412 \\
.743020 & 1.487254 & -.108049 \\
-1.320852 & -.749883 & 1.636777 \\
-2.093774 & .378897 & -1.008864 \\
2.726121 & .777927 & -.865116 \\
-.203035 & 1.012099 & 1.014705 \\
1.218116 & 2.431724 & .184135 \\
.119712 & 1.725055 & -.991090 \\
-1.057631 & 1.692484 & 1.102877
\end{array}
$$

CASPT2 $(10,10) / 6-31 G * / / U B 3 L Y P / 6-31 G$ *

Reference energy: $\quad-341.9349733043$

Total energy: $\quad-342.9218772919$ 
CASPT2 $(6,6) / 6-31 G * / / U B 3 L Y P / 6-31 G$ *

Reference energy: $\quad-341.9035497235$

Total energy: $\quad-342.9207398831$

Intermediate from 2: chair like diazenyl dirad, exo $\mathrm{N}=\mathrm{N}$

UB3LYP/6-31G(d)

$E(U B+H F-L Y P)=-344.052725893$

Sum of electronic and zero-point Energies= -343.899804

Sum of electronic and thermal Energies= $\quad-343.891742$

Sum of electronic and thermal Enthalpies $=\quad-343.890798$

Sum of electronic and thermal Free Energies= $\quad-343.932342$

KCAL/MOL
TOTAL
101.019

E (Thermal)
CV

CAL/MOL-KELVIN CAL/MOL-KELVIN

30.056
$S$ 87.438

\begin{tabular}{|c|c|c|c|c|}
\hline 1 & 6 & -1.951770 & $\odot .00 \odot \odot 24$ & -0.429146 \\
\hline 2 & 6 & -1.192688 & 1.287313 & -0.368676 \\
\hline 3 & 1 & -0.603721 & -1.318588 & 1.722914 \\
\hline 4 & 6 & -0.117977 & 1.273614 & 0.739394 \\
\hline 5 & 1 & -0.682667 & 1.467974 & -1.333512 \\
\hline 6 & 1 & -0.603685 & 1. 318572 & 1.722930 \\
\hline 7 & 1 & -1.868466 & 2.137912 & -0.218919 \\
\hline 8 & 6 & 0.733792 & $-0.0 \odot \odot \odot 2 \odot$ & 0.711392 \\
\hline 9 & 1 & 0.529602 & 2.154991 & $\odot .660154$ \\
\hline 10 & 7 & 1.539863 & $-0.000 \odot 23$ & -0.605089 \\
\hline 11 & 6 & -1.192724 & -1.287287 & -0.368691 \\
\hline 12 & 1 & 1.465607 & -0.000036 & 1.526778 \\
\hline 13 & 7 & 2.717189 & ๑. .0०००००9 & -0.615345 \\
\hline 14 & 1 & -2.978064 & 0.000041 & -0.786871 \\
\hline 15 & 6 & -0.118012 & -1.273631 & 0.739378 \\
\hline 16 & 1 & -1.868526 & -2.137868 & -0.218943 \\
\hline 17 & 1 & -0.682710 & -1.467951 & -1.333530 \\
\hline 18 & 1 & 0.529543 & -2.155025 & 0.660127 \\
\hline
\end{tabular}

Transition structure from 2: TS(C-IIb) cleave 2nd CN, chair-like geom, endo $\underline{\mathrm{N}=\mathrm{N}}$

UB3LYP/6-31G(d)

$E(U B+H F-L Y P)=-344.0503969$

Sum of electronic and zero-point Energies= -343.899721

Sum of electronic and thermal Energies= -343.891610

Sum of electronic and thermal Enthalpies= $\quad-343.890666$

Sum of electronic and thermal Free Energies= $\quad-343.932443$

$\begin{array}{rr}\mathrm{KCAL} / \mathrm{MOL} \\ \text { TOTAL } & 99.640\end{array}$
CV

CAL/MOL-KELVIN CAL/MOL-KELVIN 29.548

\section{$S$}

87.928

$\begin{array}{ll}1 & 6 \\ 2 & 6 \\ 3 & 1 \\ 4 & 6 \\ 5 & 1\end{array}$

1.756062
1.375741
$\odot .394070$
0.522584
0.776950

0.492834
-0.948146
1.130237
-1.413100
-1.118660

-0.525772
-0.635054
1.963683
0.567928
-1.549861 


$\begin{array}{rrrrr}6 & 1 & 1.168247 & -1.517414 & 1.451884 \\ 7 & 1 & 2.263089 & -1.584495 & -0.732874 \\ 8 & 6 & -0.575726 & -0.426940 & 0.894772 \\ 9 & 1 & 0.086085 & -2.399739 & 0.371187 \\ 10 & 7 & -1.735195 & -0.587673 & -0.471306 \\ 11 & 6 & 0.716653 & 1.498893 & -0.154131 \\ 12 & 1 & -1.245226 & -0.767284 & 1.687408 \\ 13 & 7 & -2.278190 & 0.338981 & -0.884565 \\ 14 & 1 & 2.713620 & 0.827740 & -0.915012 \\ 15 & 6 & -0.166136 & 1.022600 & 1.023340 \\ 16 & 1 & 1.173139 & 2.464919 & 0.092102 \\ 17 & 1 & 0.046436 & 1.693011 & -1.012347 \\ 18 & 1 & -1.057775 & 1.655679 & 1.108430\end{array}$

CASPT2 $(10,10) / 6-31 G * / / U B 3 L Y P / 6-31 G$ *

Reference energy: $\quad-341.9406909964$

Total energy: $\quad-342.9175987415$

CASPT2 $(6,6) / 6-31 G * / / U B 3 L Y P / 6-31 G$ *

Reference energy: $\quad-341.9090929205$

Total energy: $\quad-342.9164935660$

Transition structure from 2: TS(C-IIb) cleave 2nd CN, chair-like geom, exo $\underline{\mathbf{N}=\mathbf{N}}$

UB3LYP/6-31G(d)

$\mathrm{E}(\mathrm{UB}+\mathrm{HF}-\mathrm{LYP})=-344.049234152$

Sum of electronic and zero-point Energies= -343.898859

Sum of electronic and thermal Energies= -343.891495

Sum of electronic and thermal Enthalpies= -343.890550

Sum of electronic and thermal Free Energies $=\quad-343.930434$

TOTAL

E (Thermal) $\mathrm{KCAL} / \mathrm{MOL}$

CV

$S$

$\begin{array}{rrrrr}1 & 6 & -1.930 \odot 24 & .002755 & -.498968 \\ 2 & 6 & -1.173593 & 1.287880 & -.405534 \\ 3 & 1 & -.728407 & -1.389847 & 1.715044 \\ 4 & 6 & -.169921 & 1.282362 & .771846 \\ 5 & 1 & -.596141 & 1.453202 & -1.333965 \\ 6 & 1 & -.724026 & 1.388368 & 1.716979 \\ 7 & 1 & -1.853585 & 2.142731 & -.311983 \\ 8 & 6 & .626569 & -.002262 & .840132 \\ 9 & 1 & .507109 & 2.143265 & .707548 \\ 10 & 7 & 1.634388 & -.002922 & -.672174 \\ 11 & 6 & -1.177593 & -1.284843 & -.407374 \\ 12 & 1 & 1.406898 & -.003936 & 1.602556 \\ 13 & 7 & 2.779445 & .001073 & -.573070 \\ 14 & 1 & -2.956946 & .004613 & -.853781 \\ 15 & 6 & -.173979 & -1.284228 & .770062 \\ 16 & 1 & -1.860248 & -2.137715 & -.315181 \\ 17 & 1 & -.600522 & -1.450520 & -1.335974 \\ 18 & 1 & .500281 & -2.147207 & .704481\end{array}$


Product: 1,5-hexadiene

RB3LYP/6-31G(d)

$E(R B+H F-L Y P)=-234.604907363$

Sum of electronic and zero-point Energies $=\quad-234.463340$

Sum of electronic and thermal Energies= -234.457339

Sum of electronic and thermal Enthalpies= $=-234.456395$

Sum of electronic and thermal Free Energies= $\quad-234.492354$

TOTAL

E (Thermal) $\mathrm{KCAL} / \mathrm{MOL}$

92.600
CV

CAL/MOL-KELVIN CAL/MOL-KELVIN 21.911
75.682

$\begin{array}{rl}1 & 1 \\ 2 & 6 \\ 3 & 6 \\ 4 & 1 \\ 5 & 1 \\ 6 & 6 \\ 7 & 6 \\ 8 & 1 \\ 9 & 1 \\ 10 & 1 \\ 11 & 1 \\ 12 & 6 \\ 13 & 6 \\ 14 & 1 \\ 15 & 1 \\ 16 & 1\end{array}$

\begin{abstract}
$-0.311123$
$\odot .283164$

$-0.283164$

1.364392

$-1.372516$

0.464646

$-0.464646$

1.126088

1.126088

$-1.126088$

$-1.126088$

0.283164

$-0.283164$

1.372516

$-1.364392$

0.311123
\end{abstract}

4.044978

3.135272

1.928394

3. 264475

1. 862941

0.611074

$-0.611074$

0.559992

0.559992

$-0.559992$

$-0.559992$

$-1.928394$

$-3.135272$

$-1.862941$

$-3.264475$

$-4.044978$
0.000000

$\odot .000000$

0.000000

$\odot .000000$

$\odot .000000$

○. 000000

0.000000

0.876724

$-0.876724$

$-0.876724$

$\odot .876724$

0.000000

$\odot .000000$

$\odot .000000$

$\odot .000000$

๑. 000000 


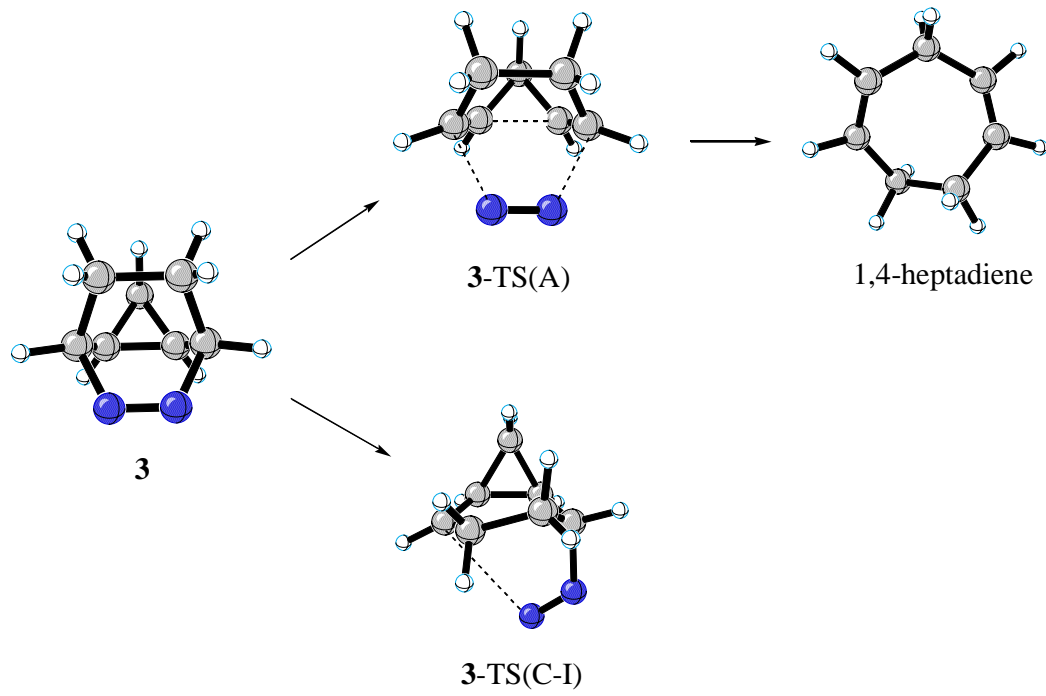

Reactant 3: exo-cyclopropane DBO

RB3LYP/6-31G(d)

$E(R B+H F-L Y P)=-382.179764103$

Sum of electronic and zero-point Energies= -382.014451

Sum of electronic and thermal Energies= $\quad-382.008199$

Sum of electronic and thermal Enthalpies $=\quad-382.007255$

Sum of electronic and thermal Free Energies= $\quad-382.044536$

$\begin{array}{cc} & \text { KCAL/MOL } \\ \text { TOTAL } & 107.659\end{array}$

CV $S$

E (Thermal)

CAL/MOL-KELVIN CAL/MOL-KELVIN

27.229

78.465

$\begin{array}{rr}1 & 6 \\ 2 & 6 \\ 3 & 6 \\ 4 & 6 \\ 5 & 1 \\ 6 & 1 \\ 7 & 1 \\ 8 & 6 \\ 9 & 1 \\ 10 & 7 \\ 11 & 6 \\ 12 & 1 \\ 13 & 7 \\ 14 & 1 \\ 15 & 6 \\ 16 & 1 \\ 17 & 1 \\ 18 & 1 \\ 19 & 1\end{array}$

$-.069913$

$-.774039$

- . 043706

$-.774039$

$-1.681591$

1. 040382

$-.512241$

- . 069913

$-1.681591$

$-.774039$

1. 395709

$-.151649$

$-.774039$

$-.151649$

1. 395709

1.972033

1.835985

1.835985

1.972033
$-.295623$ .963800

2. 048224

.963800

1. 235593

2. 088959

3. 028501

$-.295623$

1. 235593

$-1.478786$

$-.386113$

$-.420709$

$-1.478786$

$-.420709$

$-.386113$ .448277

$-1.307441$

$-1.307441$

.448277
1.253686

.756890

.000000

$-.756890$

1.289512

.000000

.000000

$-1.253686$

$-1.289512$

$-.622532$

.775727

$-2.334744$

.622532

2. 334744

$-.775727$

1.188020

1.168962

$-1.168962$

$-1.188020$

CASPT2 $(10,10) / 6-31 G * / / B 3 L Y P / 6-31 G$ *

Reference energy:

Total energy:
$-379.8223632493$

$-380.9443402022$ 
Transition structure 3-TS(A): pericyclic loss of Nitrogen

RB3LYP/6-31G(d)

$E(R B+H F-L Y P)=-382.145966550$

Sum of electronic and zero-point Energies= -381.985870

Sum of electronic and thermal Energies= $\quad-381.978588$

Sum of electronic and thermal Enthalpies= $\quad-381.977643$

Sum of electronic and thermal Free Energies= $\quad-382.016924$

\begin{tabular}{|c|c|c|c|c|c|}
\hline \multirow{2}{*}{$\begin{array}{c}\text { TOTAL } \\
1\end{array}$} & \multicolumn{2}{|r|}{$\begin{array}{c}\text { E (Thermal) } \\
\text { KCAL/MOL } \\
105.032\end{array}$} & $\mathrm{CAL} / \mathrm{MOL}$ & $\begin{array}{l}\text { ELIN } \\
13\end{array}$ & $\begin{array}{c}\mathrm{S} \\
\mathrm{AL} / \mathrm{MOL}-\mathrm{KELVIN} \\
82.672\end{array}$ \\
\hline & 6 & & .762114 & .970445 & .890512 \\
\hline 2 & 1 & & -1.862890 & -1.196066 & -1.169135 \\
\hline 3 & 1 & & -1.862890 & -1.196066 & 1.169135 \\
\hline 4 & 6 & & - . .017012 & -.127160 & 1.358624 \\
\hline 5 & 6 & & .762114 & .970445 & -.890512 \\
\hline 6 & 6 & & - . 017012 & -.127160 & -1.358624 \\
\hline 7 & 7 & & .762114 & -1.738486 & .581035 \\
\hline 8 & 6 & & -1.412616 & -.271568 & .793137 \\
\hline 9 & 1 & & .159637 & -.476939 & 2.368212 \\
\hline 10 & 1 & & .159637 & -.476939 & -2.368212 \\
\hline 11 & 6 & & -1.412616 & -.271568 & -.793137 \\
\hline 12 & 7 & & .762114 & -1.738486 & -.581035 \\
\hline 13 & 1 & & -2.046393 & .551947 & -1.146115 \\
\hline 14 & 1 & & -2.046393 & .551947 & 1.146115 \\
\hline 15 & 1 & & -.841424 & 2.233208 &.$\odot \odot \odot \odot \odot \odot$ \\
\hline 16 & 6 & & .230793 & 2.043857 & $.00000 \odot$ \\
\hline 17 & 1 & & 1.742257 & 1.130558 & 1.331451 \\
\hline 18 & 1 & & 1.742257 & 1.130558 & -1.331451 \\
\hline 19 & 1 & & .812016 & 2.962841 & $.0 \odot \odot \odot \odot \odot$ \\
\hline
\end{tabular}

CASPT2 $(10,10) / 6-31 G * / / B 3 L Y P / 6-31 G$ *

Reference energy: $\quad-379.7876018627$

Total energy: $\quad-380.9057806795$

\section{Transition structure 3-TS(C-I): 1-bond CN cleavage}

UB3LYP/6-31G(d)

$E(U B+H F-L Y P)=-382.122193894$

Sum of electronic and zero-point Energies= $\quad-381.964193$

Sum of electronic and thermal Energies= -381.956363

Sum of electronic and thermal Enthalpies= $\quad-381.955419$

Sum of electronic and thermal Free Energies= $\quad-381.996141$

$\begin{array}{cccc} & \text { KCAL/MOL } & \text { CAL/MOL-KELVIN } & \text { CAL/MOL-KELVIN } \\ \text { TOTAL } & 104.060 & 30.953 & 85.707\end{array}$

$\begin{array}{rrrrr}1 & 6 & -0.543610 & -0.695416 & 0.852615 \\ 2 & 7 & -1.829972 & -0.862051 & -0.057692 \\ 3 & 7 & -1.859208 & -0.475727 & -1.167042 \\ 4 & 6 & 0.640541 & 1.152135 & -0.980040 \\ 5 & 1 & 0.675405 & 1.669047 & -1.933550\end{array}$




$\begin{array}{rrrrr}6 & 6 & -0.195375 & 1.744459 & 0.117184 \\ 7 & 1 & 0.306895 & 0.848342 & 2.050837 \\ 8 & 6 & 0.672129 & -1.166379 & 0.098286 \\ 9 & 1 & -0.767170 & -1.340353 & 1.705732 \\ 10 & 6 & 1.292227 & -0.154502 & -0.872703 \\ 11 & 1 & -1.149402 & 2.081496 & -0.312495 \\ 12 & 1 & 0.267958 & 2.658261 & 0.526925 \\ 13 & 6 & -0.477893 & 0.770905 & 1.288612 \\ 14 & 1 & -1.414205 & 1.043497 & 1.788181 \\ 15 & 6 & 2.036427 & -0.598659 & 0.385839 \\ 16 & 1 & 2.883998 & -1.264164 & 0.246231 \\ 17 & 1 & 2.166116 & 0.116222 & 1.192638 \\ 18 & 1 & 0.628731 & -2.204373 & -0.225698 \\ 19 & 1 & 1.679251 & -0.558790 & -1.804416\end{array}$

CASPT2 $(10,10) / 6-31 G * / / U B 3 L Y P / 6-31 G$ *

Reference energy: $\quad-379.7673771392$

Total energy: $\quad-380.8833431746$ 

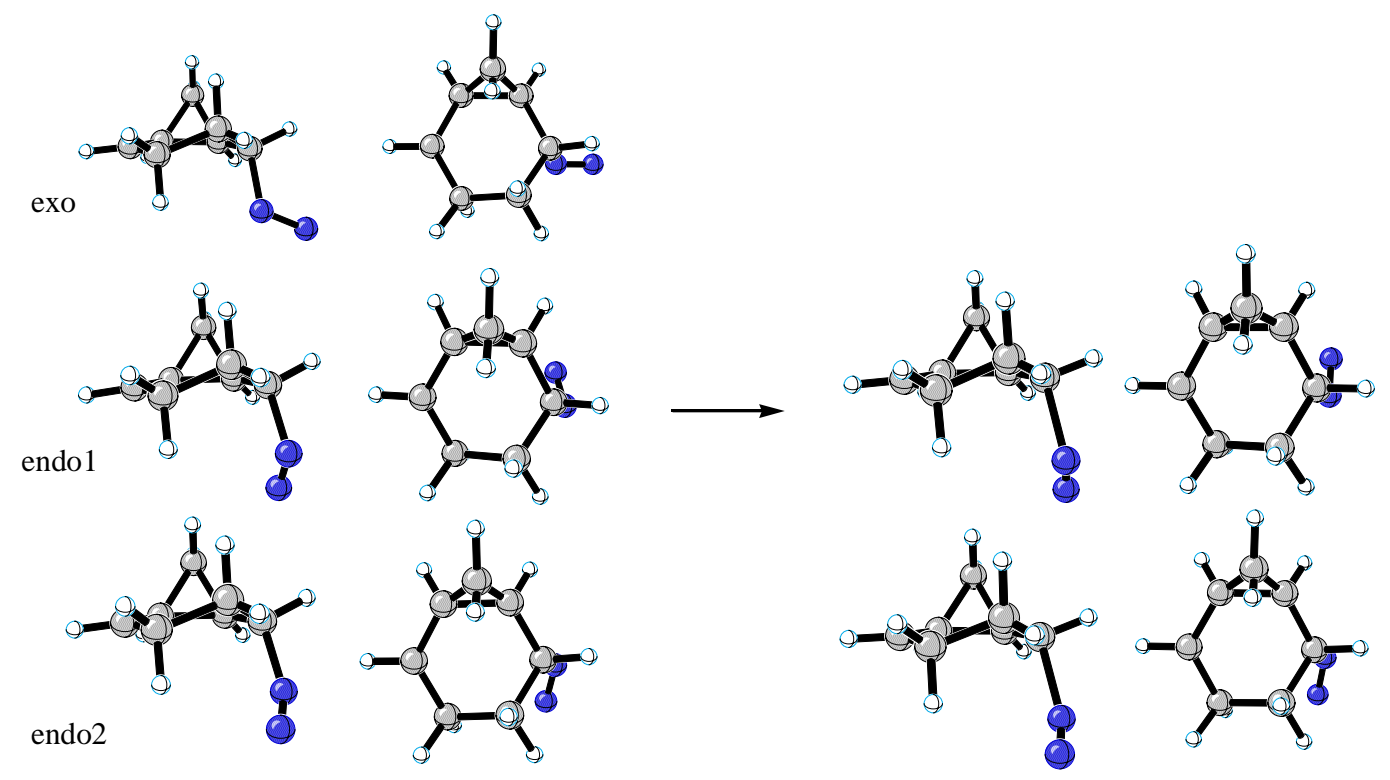

diazenyl diradical

3-TS(C-II)

Intermediate from 3: exp-prop diazenyl dirad, endo1 $\mathrm{N}=\mathrm{N}$

UB3LYP/6-31G(d)

$E(U B+H F-L Y P)=-382.125410543$

Sum of electronic and zero-point Energies= $\quad-381.967299$

Sum of electronic and thermal Energies= $\quad-381.958720$

Sum of electronic and thermal Enthalpies= $\quad-381.957776$

Sum of electronic and thermal Free Energies= $\quad \mathbf{- 3 8 2 . 0 0 0 6 3 5}$

TOTAL

E (Thermal) $\mathrm{KCAL} / \mathrm{MOL}$ 104.600
CV

$\mathrm{CAL} / \mathrm{MOL}-\mathrm{KELVIN}$ 32.768
$S$

CAL/MOL-KELVIN 90.204

$\begin{array}{rl}1 & 6 \\ 2 & 7 \\ 3 & 7 \\ 4 & 6 \\ 5 & 1 \\ 6 & 6 \\ 7 & 1 \\ 8 & 6 \\ 9 & 1 \\ 10 & 6 \\ 11 & 1 \\ 12 & 1 \\ 13 & 6 \\ 14 & 1 \\ 15 & 6 \\ 16 & 1 \\ 17 & 1 \\ 18 & 1 \\ 19 & 1\end{array}$

$-0.215590$

$-0.040183$

$-0.653842$

0.824239

1. 208381

1.766605

1.140567

$-1.186672$

$-0.610442$

$-0.629543$

2. 014081

2.725738

1.190185

1.843278

$-1.285163$

$-2.265157$

$-0.637303$

$-2.109981$

$-1.235351$
0.815183

$-0.020927$

$-0.993516$

$-0.908318$

$-1.650620$

$-0.254147$

1.824240

0.159250

1.763213

$-0.726499$

$-0.954600$

$-0.028364$

1.029003

1.390298

$\odot .630289$

0.615946

1.431696

$-0.172313$

$-1.586952$

Intermediate from 3: exp-prop diazenyl dirad, endo2 $\mathrm{N}=\mathrm{N}$ 
UB3LYP/6-31G(d)

$\mathrm{E}(\mathrm{UB}+\mathrm{HF}-\mathrm{LYP})=-382.125254174$

Sum of electronic and zero-point Energies= -381.967134

Sum of electronic and thermal Energies= -381.958615

Sum of electronic and thermal Enthalpies= $\quad-381.957671$

Sum of electronic and thermal Free Energies= $\quad-382.000189$

$\begin{array}{cr}\text { KCAL/MOL } \\ \text { TOTAL } & 104.567\end{array}$

E (Thermal)
CV

$\mathrm{CAL} / \mathrm{MOL}-\mathrm{KELVIN}$

32.786
$S$

CAL/MOL-KELVIN 89.485

$\begin{array}{rrrrr}1 & 6 & -.608811 & -.717223 & .591898 \\ 2 & 7 & -1.792239 & -.927766 & -.415826 \\ 3 & 7 & -2.597023 & -.104947 & -.648476 \\ 4 & 6 & 1.167539 & 1.297212 & -.640635 \\ 5 & 1 & 1.739817 & 2.033305 & -1.198320 \\ 6 & 6 & -.069641 & 1.731563 & .081062 \\ 7 & 1 & .121054 & .720898 & 1.996754 \\ 8 & 6 & .642420 & -1.170804 & -.128104 \\ 9 & 1 & -.864173 & -1.425660 & 1.389042 \\ 10 & 6 & 1.537792 & -.118868 & -.760816 \\ 11 & 1 & -.891719 & 1.894604 & -.639825 \\ 12 & 1 & .084934 & 2.706345 & .564318 \\ 13 & 6 & -.552334 & .713712 & 1.130461 \\ 14 & 1 & -1.544860 & .995605 & 1.497988 \\ 15 & 6 & 1.998107 & -.922478 & .470516 \\ 16 & 1 & 2.763437 & -1.680085 & .321330 \\ 17 & 1 & 2.072907 & -.390869 & 1.414484 \\ 18 & 1 & .515610 & -2.101701 & -.676005 \\ 19 & 1 & 2.037390 & -.402143 & -1.685940\end{array}$

Intermediate from 3: exo-prop diazenyl dirad, exo $\mathrm{N}=\mathrm{N}$

UB3LYP/6-31G(d)

$\mathrm{E}(\mathrm{UB}+\mathrm{HF}-\mathrm{LYP})=-382.125552823$

Sum of electronic and zero-point Energies= $\quad-381.967416$

Sum of electronic and thermal Energies= -381.958835

Sum of electronic and thermal Enthalpies= -381.957891

Sum of electronic and thermal Free Energies $=\quad-382.000842$ $\begin{array}{cc}\text { TOTAL } & 104.617\end{array}$

E (Thermal)

$\begin{array}{rl}1 & 6 \\ 2 & 6 \\ 3 & 1 \\ 4 & 6 \\ 5 & 1 \\ 6 & 1 \\ 7 & 6 \\ 8 & 6 \\ 9 & 1 \\ 10 & 1 \\ 11 & 6 \\ 12 & 1\end{array}$

CV

CAL/MOL-KELVIN 32.762
$S$

CAL/MOL-KELVIN 90.398
1.630857
.858271
$-.697939$
.591564
1.760810
$-.109603$
$-.198615$
$-2.075949$
$-.524886$
$-.228222$
1. 084261
1. 004093
1.048609
2. 681577
.277382
$-.110289$
2. 086946
$-.898663$
1.568394
$-1.401877$
.620979
$-.792415$
-. 273767
.573683
.397651
.928116
1.891584
1.798993
$-.852466$
1.528869
1.469564
$-.601744$
2. 010342
$-2.393857$
$-.690674$
.573789 


$\begin{array}{rrrrr}13 & 1 & 2.443051 & 1.289498 & -1.276250 \\ 14 & 6 & .233500 & -1.201754 & -.042378 \\ 15 & 1 & 1.862833 & -1.127623 & -1.559253 \\ 16 & 1 & -1.055643 & 1.731847 & 1.316238 \\ 17 & 1 & -1.320608 & -.764090 & 1.399992 \\ 18 & 7 & -1.860473 & .006070 & -.521931 \\ 19 & 7 & -2.956066 & -.413098 & -.432036\end{array}$

Transition structure 3-TS(C-II): cleave 2nd CN bond, endo1 N=N

\begin{tabular}{|c|c|c|c|c|c|c|}
\hline \multicolumn{7}{|c|}{ UB3LYP/6-31G(d) } \\
\hline $\mathrm{E}(\mathrm{UB}+\mathrm{H}$ & $F-L Y P)=-$ & 382.124033178 & & & & \\
\hline Sum of & electronic & and zero-point & hergies= & & -381 & 967849 \\
\hline Sum of & electronic & and thermal En & yies $=$ & & -381 & 959270 \\
\hline Sum of & electronic & and thermal En & alpies= & & -381 & 958326 \\
\hline Sum of & electronic & and thermal $\mathrm{Fr}$ & Energies= & & -382 & $\odot \odot 157 \odot$ \\
\hline & & E (Thermal) & & & & S \\
\hline & & KCAL/MOL & $\mathrm{CAL} / \mathrm{MOL}$ & ELVIN & & /MOL - KELVIN \\
\hline TOTAL & & 103.390 & & อ34 & & 91.015 \\
\hline 1 & 6 & & 0.155096 & -1.17 & 862 & 0.185317 \\
\hline 2 & 6 & & -0.687052 & -0.13 & 528 & 0.857503 \\
\hline 3 & 6 & & 1.272838 & -0.70 & 275 & -0.731951 \\
\hline 4 & 6 & & 1.473400 & 0.73 & 738 & -0.927081 \\
\hline 5 & 7 & & -2.512291 & -0.68 & 1760 & -0.843940 \\
\hline 6 & 1 & & 2.211944 & 1.06 & 5374 & -1.652673 \\
\hline 7 & 6 & & 0.563373 & 1.74 & 1462 & $-\odot .298061$ \\
\hline 8 & 1 & & -1.174030 & -0.49 & 3156 & 1.769386 \\
\hline 9 & 7 & & -2.130736 & 0.12 & 9776 & -0.117626 \\
\hline 10 & 6 & & -0.077883 & 1.24 & 2294 & 1.010139 \\
\hline 11 & 1 & & 1.095769 & 2.68 & 857 & -0.112743 \\
\hline 12 & 1 & & -0.254304 & 2.00 & 2683 & -0.996933 \\
\hline 13 & 1 & & 0.679305 & 1.20 & 7697 & 1.806329 \\
\hline 14 & 1 & & -0.854391 & 1.93 & 301 & 1.345289 \\
\hline 15 & 6 & & 1.588150 & -1.37 & 3119 & 0.617044 \\
\hline 16 & 1 & & 1.982659 & -2.38 & 295 & 0.602047 \\
\hline 17 & 1 & & 1.993045 & -0.74 & 4937 & 1.405060 \\
\hline 18 & 1 & & 1.481277 & -1.33 & 3396 & -1.594761 \\
\hline 19 & 1 & & -0.387612 & -2.06 & 5497 & -0.117502 \\
\hline
\end{tabular}

CASPT2 $(10,10) / 6-31 G * / / U B 3 L Y P / 6-31 G$ *

Reference energy: $\quad-379.7744024080$

Total energy: $\quad-380.8812941172$

Transition structure $3-\mathrm{TS}(\mathrm{C}-\mathrm{II})$ : cleave 2nd CN, endo2 $\mathrm{N}=\mathrm{N}$

UB3LYP/6-31G(d)

$\mathrm{E}(\mathrm{UB}+\mathrm{HF}-\mathrm{LYP})=-382.123908316$

Sum of electronic and zero-point Energies= -381.967583

Sum of electronic and thermal Energies= -381.959101

Sum of electronic and thermal Enthalpies= $\quad-381.958157$

Sum of electronic and thermal Free Energies $=\quad-382.000934$ 
TOTAL

E (Thermal) 103.418
CV CAL/MOL-KELVIN CAL/MOL-KELVIN 31.972

90.031

$\begin{array}{rrr}0.653865 & -1.172583 & -0.104117 \\ -0.545825 & -0.695122 & 0.651060 \\ 1.547444 & -0.143384 & -0.771580 \\ 1.199845 & 1.279568 & -0.653106 \\ -2.704146 & -0.109447 & -0.576550 \\ 1.803539 & 2.009292 & -1.185732 \\ -0.043880 & 1.730255 & 0.046641 \\ -0.873289 & -1.399963 & 1.419955 \\ -1.887560 & -0.918496 & -0.473052 \\ -0.527140 & 0.743681 & 1.127561 \\ 0.101545 & 2.721866 & 0.495947 \\ -0.863622 & 1.860779 & -0.683459 \\ 0.129845 & 0.804718 & 2.006942 \\ -1.531203 & 1.022629 & 1.467649 \\ 2.037475 & -0.936405 & 0.451768 \\ 2.786494 & -1.707938 & 0.290171 \\ 2.141652 & -0.397120 & 1.388647 \\ 2.014327 & -0.444160 & -1.708645 \\ 0.501947 & -2.110556 & -0.633625\end{array}$

Product: 1,4-cycloheptadiene

RB3LYP/6-31G(d)

$E(R B+H F-L Y P)=-272.721485894$

Sum of electronic and zero-point Energies= $\quad-272.569836$

Sum of electronic and thermal Energies= -272.563288

Sum of electronic and thermal Enthalpies= $\quad-272.562344$

Sum of electronic and thermal Free Energies= $\quad-272.600732$

TOTAL

E (Thermal)

$\mathrm{KCAL} / \mathrm{MOL}$

99.271
CV

CAL/MOL-KELVIN 24.783
$S$

CAL/MOL - KELVIN 80.795

$$
\begin{array}{rrr}
2.393636 & -1.197188 & .139687 \\
1.449163 & -.684515 & .362379 \\
-.974448 & 1.310064 & .157490 \\
-.983026 & -1.303789 & -.160096 \\
-1.711977 & .005079 & .002551 \\
.309247 & -1.605532 & -.000221 \\
1.455555 & .675179 & -.359448 \\
.319579 & 1.603669 & -.003659 \\
1.466236 & .497915 & -1.444907 \\
-2.401975 & .118758 & -.851831 \\
.600452 & 2.648810 & .127896 \\
2.401873 & 1.181984 & -.131005 \\
1.454240 & -.506983 & 1.447815 \\
-1.637881 & 2.139731 & .406366 \\
-1.651374 & -2.128066 & -.413595 \\
-2.394514 & -.104596 & .863542 \\
.584750 & -2.651293 & -.137945
\end{array}
$$




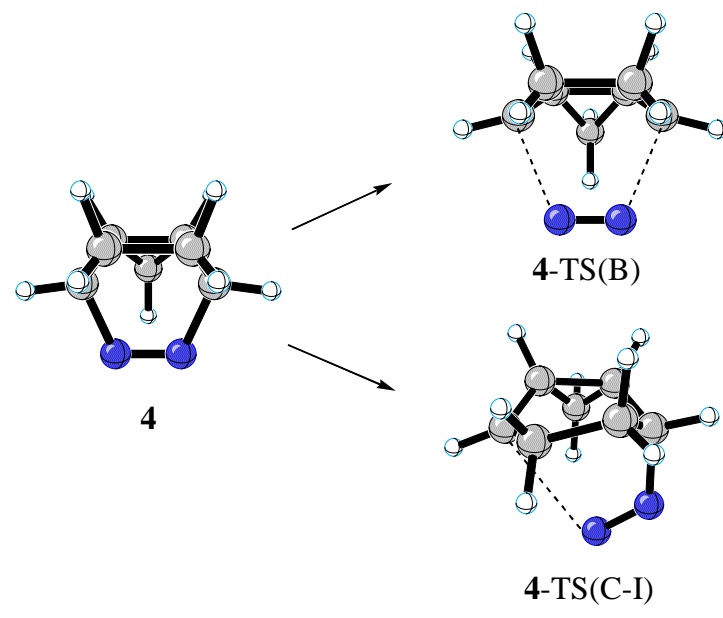

Reactant 4: endo-cyclopropane DBO

RB3LYP/6-31G(d)

$E(R B+H F-L Y P)=-382.180807393$

Sum of electronic and zero-point Energies= -382.015885

Sum of electronic and thermal Energies= -382.009503

Sum of electronic and thermal Enthalpies= $=-382.008558$

Sum of electronic and thermal Free Energies $=\quad-382.046174$

$\begin{array}{lr}\text { KCAL/MOL } \\ \text { TOTAL } & 107.495\end{array}$

E (Thermal)

$\begin{array}{rr}1 & 6 \\ 2 & 6 \\ 3 & 1 \\ 4 & 6 \\ 5 & 1 \\ 6 & 1 \\ 7 & 1 \\ 8 & 6 \\ 9 & 1 \\ 10 & 7 \\ 11 & 6 \\ 12 & 1 \\ 13 & 7 \\ 14 & 1 \\ 15 & 6 \\ 16 & 1 \\ 17 & 1 \\ 18 & 6 \\ 19 & 1\end{array}$

CV

CAL/MOL-KELVIN CAL/MOL-KELVIN

27.293

$S$

79.169
$-.217852$

$-1.498472$

1.214785

$-1.498472$

$-2.382537$

$-1.495391$

2. 184305

$-.217852$

$-2.382537$

$-.251719$

.994331

$-.209684$

$-.251719$

$-.209684$

.994331

1. 214785

3. 071447

2. 112982

$-1.495391$
1.254468

.775338

$-1.308223$

$-.775338$

1.173253

1.180718

.000000

$-1.254468$

$-1.173253$

$-.625612$

.753385

$-2.337198$

.625612

2.337198

$-.753385$

1.308223

.000000

.000000

$-1.180718$

CASPT2 $(10,10) / 6-31 G * / / B 3 L Y P / 6-31 G$ *

Reference energy: $\quad-379.8230484631$

Total energy:

$-380.9458617009$

CASPT2 $(6,6) / 6-31 G * / / B 3 L Y P / 6-31 G$ *

Reference energy:

$-379.7898814198$

Total energy:

$-380.9456250512$ 
Transition structure 4-TS(B): 2-bond CN cleavage

\begin{tabular}{|c|c|c|c|c|c|c|}
\hline \multicolumn{7}{|c|}{ UB3LYP/6-31G(d) } \\
\hline $\mathrm{E}(\mathrm{UB}+\mathrm{HF}$ & - LYP $)=-3$ & 382.106702179 & & & & \\
\hline Sum of & electronic & and zero-point & hergies= & & -381 & 950431 \\
\hline Sum of & electronic & and thermal En & yies= & & -381 & 943135 \\
\hline Sum of & electronic & and thermal En & alpies= & & -381 & 942191 \\
\hline Sum of & electronic & and thermal Fr & Energies= & & -381 & 981865 \\
\hline & & E (Thermal) & & & & $\mathrm{S}$ \\
\hline & & $\mathrm{KCAL} / \mathrm{MOL}$ & $\mathrm{CAL} / \mathrm{MOL}$ & ELVIN & & MOL-KELVIN \\
\hline TOTAL & & 102.640 & & 385 & & 83.501 \\
\hline 1 & 6 & & -0.182865 & -0.18 & 582 & 1.364795 \\
\hline 2 & 7 & & 1.776496 & -0.38 & 031 & 0.573242 \\
\hline 3 & 7 & & 1.776496 & -0.38 & 031 & -0.573242 \\
\hline 4 & 6 & & -0.182865 & -0.18 & 582 & -1.364795 \\
\hline 5 & 1 & & 0.086077 & -0.17 & 102 & -2.417910 \\
\hline 6 & 1 & & -1.598914 & 1.48 & 351 & -1.274031 \\
\hline 7 & 6 & & -0.727302 & -1.46 & 106 & -0.787740 \\
\hline 8 & 1 & & -1.761732 & -1.60 & 765 & 1.139432 \\
\hline 9 & 6 & & -0.727302 & 1.07 & 827 & 0.760427 \\
\hline 10 & 1 & & 0.086077 & -0.17 & 102 & 2.417910 \\
\hline 11 & 6 & & -0.727302 & 1.07 & 827 & $-\odot .760427$ \\
\hline 12 & 6 & & 0.116316 & 2.07 & 094 & 0.000000 \\
\hline 13 & 1 & & -1.598914 & 1.48 & 351 & 1.274031 \\
\hline 14 & 1 & & -0.245258 & 3.10 & 590 & $\odot .00 \odot \odot \odot \odot$ \\
\hline 15 & 1 & & -0.159810 & -2.31 & 417 & -1.174286 \\
\hline 16 & 1 & & -1.761732 & -1.60 & 765 & -1.139432 \\
\hline 17 & 6 & & -0.727302 & -1.46 & 106 & 0.787740 \\
\hline 18 & 1 & & -0.159810 & -2.31 & 417 & 1.174286 \\
\hline 19 & 1 & & 1.194796 & 1.98 & 480 & $\odot . \odot \odot \odot \odot \odot \odot$ \\
\hline
\end{tabular}

CASPT2 $(10,10) / 6-31 G * / / U B 3 L Y P / 6-31 G *$

Reference energy: $\quad-379.7677906884$

Total energy: -380.8703360488

CASPT2 $(6,6) / 6-31 G * / / B 3 L Y P / 6-31 G *$

Reference energy: $\quad-379.7337362539$

Total energy: $\quad-380.8695865562$

Transition structure 4-TS(C-I): 1-bond CN cleavage

UB3LYP/ $6-31 G(d)$

$E(U B+H F-L Y P)=-382.115443652$

Sum of electronic and zero-point Energies= $\quad-381.956696$

Sum of electronic and thermal Energies= $\quad-381.949308$

Sum of electronic and thermal Enthalpies= $\quad-381.948363$

Sum of electronic and thermal Free Energies= $\quad-381.987978$

$\begin{array}{cccc} & \mathrm{KCAL} / \mathrm{MOL} & \mathrm{CAL} / \mathrm{MOL}-\mathrm{KELVIN} & \mathrm{CAL} / \mathrm{MOL}-\mathrm{KELVIN} \\ \text { TOTAL } & 104.252 & 30.034 & 83.375\end{array}$ 


\begin{tabular}{|c|c|c|c|c|}
\hline & & & & \\
\hline 1 & 6 & -0.398444 & -0.776209 & 0.919903 \\
\hline 2 & 7 & -0.681026 & -1.680906 & -0.281328 \\
\hline 3 & 7 & -0.494079 & -1.319913 & -1.391479 \\
\hline 4 & 6 & 0.112574 & 1.329593 & -0.851825 \\
\hline 5 & 1 & $\odot .289672$ & 1.806474 & -1.812851 \\
\hline 6 & 1 & 1.853771 & 1.822623 & 0.406536 \\
\hline 7 & 6 & -1.296834 & 1.272562 & $-\odot .328758$ \\
\hline 8 & 1 & -1.251399 & $\odot .943374$ & 1.830346 \\
\hline 9 & 6 & 1.015586 & -0.190098 & 0.942252 \\
\hline 10 & 1 & $-\odot .550290$ & -1.423402 & 1.786459 \\
\hline 11 & 6 & 1.258389 & 0.989848 & $\odot .019572$ \\
\hline 12 & 6 & 2.021927 & -0.311338 & -0.172393 \\
\hline 13 & 1 & 1.423437 & -0.138161 & 1.949940 \\
\hline 14 & 1 & 3.067363 & -0.325411 & 0.125141 \\
\hline 15 & 1 & -1.964896 & 0.922277 & -1.126571 \\
\hline 16 & 1 & -1.663944 & 2.281146 & $-\odot .068740$ \\
\hline 17 & 6 & -1.433103 & $\odot .363889$ & 0.916399 \\
\hline 18 & 1 & -2.449445 & -0.038820 & $\odot .991787$ \\
\hline 19 & 1 & 1.790895 & -0.913841 & -1.043309 \\
\hline CASPT & $-31 G * / / U E$ & & & \\
\hline & e energy: & 7612054826 & & \\
\hline & ergy: & 8794874467 & & \\
\hline CASPT & $31 G * / / U B 3 L$ & & & \\
\hline & e energy: & 7280761008 & & \\
\hline & ergy: & 8789114844 & & \\
\hline
\end{tabular}



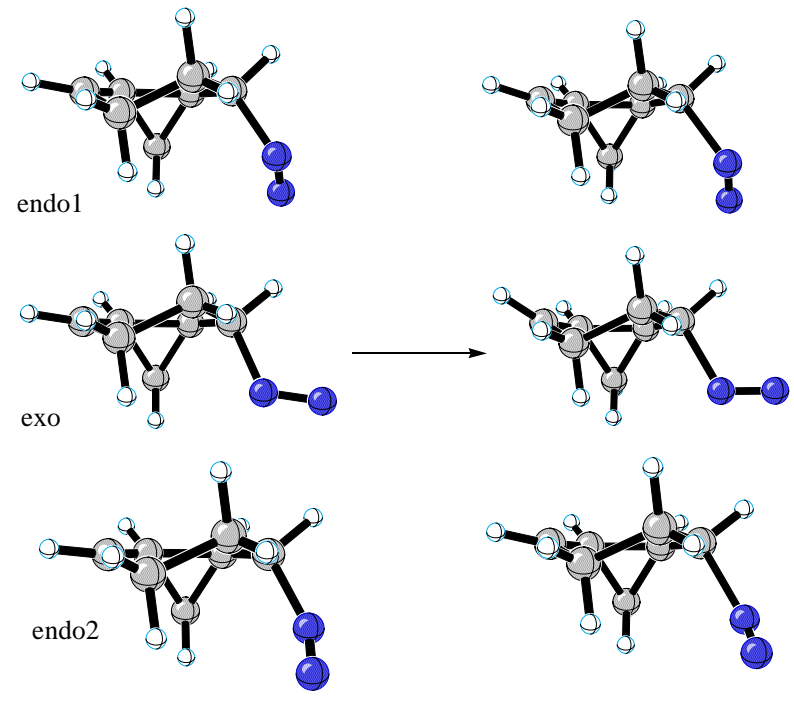

diazenyl diradical

4-TS(C-II)

Intermediate from 4: endo prop diazenyl dirad, endo1 $\mathrm{N}=\mathrm{N}$

UB3LYP/ $6-31 G(d)$

$\mathrm{E}(\mathrm{UB}+\mathrm{HF}-\mathrm{LYP})=-382.122318736$

Sum of electronic and zero-point Energies= -381.964533

Sum of electronic and thermal Energies= $\quad-381.955807$

Sum of electronic and thermal Enthalpies= $\quad-381.954863$

Sum of electronic and thermal Free Energies $=\quad-381.997880$

$\begin{array}{cc} & \text { KCAL/MOL } \\ \text { TOTAL } & 104.488\end{array}$

$$
\text { E (Thermal) }
$$

$\begin{array}{rl}1 & 6 \\ 2 & 6 \\ 3 & 1 \\ 4 & 6 \\ 5 & 1 \\ 6 & 1 \\ 7 & 6 \\ 8 & 6 \\ 9 & 1 \\ 10 & 1 \\ 11 & 6 \\ 12 & 1 \\ 13 & 1 \\ 14 & 1 \\ 15 & 6 \\ 16 & 1 \\ 17 & 1 \\ 18 & 7 \\ 19 & 7\end{array}$

CV CAL/MOL-KELVIN CAL/MOL-KELVIN 33.195
$S$ 90.538

$\begin{array}{rrr}.715786 & -.449467 & .773948 \\ -.228319 & -1.606098 & .424506 \\ -1.958651 & 1.838540 & .807833 \\ -1.251371 & -1.252567 & -.673914 \\ .371716 & -2.476511 & .136721 \\ -.778903 & -1.874436 & 1.335037 \\ -.145102 & 1.704593 & -.479152 \\ -2.042480 & -.045356 & -.276598 \\ -.711325 & -1.091697 & -1.625747 \\ .121117 & 1.246304 & -1.425812 \\ .092967 & .933490 & .786085 \\ -3.106245 & .015472 & -.481737 \\ -.000161 & 2.780747 & -.447380 \\ 1.176186 & -.655222 & 1.745378 \\ -1.346612 & 1.116739 & .271149 \\ .365316 & 1.538705 & 1.647468 \\ -1.913192 & -2.108265 & -.849063 \\ 1.983327 & -.572795 & -.175872 \\ 2.540234 & .341132 & -.652535\end{array}$

$\operatorname{CASPT2}(10,10) / 6-31 G * / / U B 3 L Y P / 6-31 G$ * 
Reference energy: $\quad-379.7666312133$

Total energy: $\quad-380.8810378975$

CASPT2 $(6,6) / 6-31 G * / / U B 3 L Y P / 6-31 G *$

Reference energy: $\quad-379.7333315809$

Total energy: $\quad-380.8804213272$

Intermediate from 4: endo prop diazenyl dirad, exo $\mathrm{N}=\mathrm{N}$

UB3LYP/6-31G(d)

$E(U B+H F-L Y P)=-382.123847904$

Sum of electronic and zero-point Energies= $\quad-381.965641$

Sum of electronic and thermal Energies= $\quad-381.957037$

Sum of electronic and thermal Enthalpies= -381.956093

Sum of electronic and thermal Free Energies= $\quad-381.999026$

$\begin{array}{cccc} & \text { E (Thermal) } & \text { CV } & \text { S } \\ \text { KCAL/MOL } & \text { CAL/MOL-KELVIN } & \text { CAL/MOL-KELVIN } \\ \text { TOTAL } & 104.675 & 32.867 & 90.360\end{array}$

$\begin{array}{rrrrr}1 & 6 & -0.799425 & 0.134231 & 0.682562 \\ 2 & 6 & -0.124529 & 1.501290 & 0.513838 \\ 3 & 1 & 2.353014 & -1.441926 & 0.777323 \\ 4 & 6 & 0.996005 & 1.500060 & -0.544451 \\ 5 & 1 & -0.885616 & 2.253873 & 0.276589 \\ 6 & 1 & 0.306983 & 1.772880 & 1.485904 \\ 7 & 6 & 0.618754 & -1.691002 & -0.588237 \\ 8 & 6 & 2.025848 & 0.464009 & -0.215985 \\ 9 & 1 & 0.543295 & 1.326742 & -1.537312 \\ 10 & 1 & 0.269214 & -1.267766 & -1.524318 \\ 11 & 6 & 0.152858 & -1.054230 & 0.692737 \\ 12 & 1 & 3.078533 & 0.654110 & -0.399300 \\ 13 & 1 & 0.748141 & -2.769806 & -0.598896 \\ 14 & 1 & -1.409087 & 0.130238 & 1.591388 \\ 15 & 6 & 1.605467 & -0.861232 & 0.240345 \\ 16 & 1 & -0.001167 & -1.735651 & 1.525496 \\ 17 & 1 & 1.454666 & 2.494453 & -0.594801 \\ 18 & 7 & -1.802389 & -0.035727 & -0.501290 \\ 19 & 7 & -2.955874 & -0.160830 & -0.311128\end{array}$

Intermediate from 4: endo prop diazenyl dirad, endo2 $\mathrm{N}=\mathrm{N}$

UB3LYP/6-31G(d)

$E(U B+H F-L Y P)=-382.1229069$

Sum of electronic and zero-point Energies= $\quad-381.964768$

Sum of electronic and thermal Energies= -381.956153

Sum of electronic and thermal Enthalpies= $\quad-381.955209$

Sum of electronic and thermal Free Energies= $\quad-381.998218$
6
6
.869951
.720959

E (Thermal)

104.639
CV

CAL/MOL-KELVIN

32.848
$\mathrm{S}$

CAL/MOL - KELVIN

90.519 


$\begin{array}{rrrrr}3 & 1 & 2.689540 & -.866283 & .491318 \\ 4 & 6 & .445590 & 1.647767 & -.450245 \\ 5 & 1 & -1.450792 & 1.819839 & .613664 \\ 6 & 1 & -.032897 & 1.644500 & 1.655854 \\ 7 & 6 & .921740 & -1.535967 & -.673384 \\ 8 & 6 & 1.741479 & .908747 & -.332412 \\ 9 & 1 & -.073721 & 1.417607 & -1.398429 \\ 10 & 1 & .352686 & -1.194625 & -1.533110 \\ 11 & 6 & .478172 & -1.091702 & .693838 \\ 12 & 1 & 2.666076 & 1.362915 & -.675350 \\ 13 & 1 & 1.329740 & -2.538019 & -.774290 \\ 14 & 1 & -1.198088 & -.407200 & 1.846446 \\ 15 & 6 & 1.749990 & -.494723 & .086314 \\ 16 & 1 & .627239 & -1.816003 & 1.490443 \\ 17 & 1 & .622371 & 2.729888 & -.468260 \\ 18 & 7 & -1.865877 & -.726090 & -.101114 \\ 19 & 7 & -2.434795 & -.035738 & -.861516\end{array}$

Transition structure 4-TS(C-II): cleave 2nd $\mathrm{CN}$, endo1 $\mathrm{N}=\mathrm{N}$

UB3LYP/6-31G(d)

$\mathrm{E}(\mathrm{UB}+\mathrm{HF}-\mathrm{LYP})=-382.122018282$

Sum of electronic and zero-point Energies= $\quad-381.965565$

Sum of electronic and thermal Energies= -381.957000

Sum of electronic and thermal Enthalpies= $-\mathbf{3 8 1 . 9 5 6 0 5 6}$

Sum of electronic and thermal Free Energies= $\quad-381.998991$

\begin{tabular}{|c|c|c|c|c|c|}
\hline \multirow{2}{*}{$\begin{array}{c}\text { TOTAL } \\
1\end{array}$} & \multicolumn{2}{|r|}{$\begin{array}{c}\text { E (Thermal) } \\
\text { KCAL/MOL } \\
103.550\end{array}$} & $\mathrm{CAL} / \mathrm{MOL}$ & $\begin{array}{l}\text { LVIN } \\
81\end{array}$ & $\begin{array}{c}\mathrm{S} \\
\mathrm{CAL} / \mathrm{MOL}-\mathrm{KELVIN} \\
90.365\end{array}$ \\
\hline & 6 & & 0.071000 & 0.936089 & 0.791354 \\
\hline 2 & 6 & & $\odot .672809$ & -0.439632 & $\odot .817423$ \\
\hline 3 & 6 & & -1.373581 & 1.115309 & 0.249386 \\
\hline 4 & 6 & & -2.057770 & -0.061184 & $-\odot .273318$ \\
\hline 5 & 7 & & 2.560838 & 0.359425 & -0.657171 \\
\hline 6 & 1 & & -3.139305 & -0.065955 & -0.351461 \\
\hline 7 & 6 & & -1.244745 & -1.246117 & $-\odot .693435$ \\
\hline 8 & 1 & & 1.134316 & -0.646457 & 1.785808 \\
\hline 9 & 7 & & 2.067364 & -0.558357 & $-\odot .148129$ \\
\hline 10 & 6 & & -0.220766 & -1.606495 & 0.404859 \\
\hline 11 & 1 & & -1.888609 & -2.109820 & -0.891729 \\
\hline 12 & 1 & & -0.700877 & -1.051329 & -1.635469 \\
\hline 13 & 1 & & -0.773388 & -1.932919 & 1.296645 \\
\hline 14 & 1 & & 0.409210 & -2.447182 & $\odot .092147$ \\
\hline 15 & 6 & & -0.161429 & 1.681381 & -0.491271 \\
\hline 16 & 1 & & 0.116425 & 1.206758 & -1.426293 \\
\hline 17 & 1 & & -0.015083 & 2.757620 & $-\odot .476026$ \\
\hline 18 & 1 & & 0.334881 & 1.561305 & 1.641087 \\
\hline 19 & 1 & & -1.988095 & 1.844395 & $\odot .77240 \odot$ \\
\hline
\end{tabular}

CASPT2 $(10,10) / 6-31 G * / / U B 3 L Y P / 6-31 G *$

Reference energy: $\quad-379.7691239638$

Total energy: -380.8800015945

CASPT2 $(6,6) / 6-31 G * / / U B 3 L Y P / 6-31 G *$ 
Reference energy: $\quad-379.7352396453$

Total energy: $\quad-380.8794881352$

\section{Transition structure 4-TS(C-II): cleave 2nd CN, exo $\mathrm{N}=\mathrm{N}$}

UB3LYP/6-31G(d)

$E(U B+H F-L Y P)=-382.122499408$

Sum of electronic and zero-point Energies= -381.966176

Sum of electronic and thermal Energies= $\quad-381.957575$

Sum of electronic and thermal Enthalpies= -381.956631

Sum of electronic and thermal Free Energies= $\quad-381.999835$

$\begin{array}{cc} & \text { KCAL/MOL } \\ \text { TOTAL } & 103.491\end{array}$

E (Thermal)
CV $S$

$$
\begin{array}{cc}
\mathrm{CAL} / \mathrm{MOL}-\mathrm{KELVIN} & \mathrm{CAL} / \mathrm{MOL}-\mathrm{KELVIN} \\
32.236 & 90.930
\end{array}
$$

Transition structure 4-TS(C-II): cleave 2nd $\mathrm{CN}$, endo2 $\mathrm{N}=\mathrm{N}$

UB3LYP/6-31G(d)

$E(U B+H F-L Y P)=-382.122078967$

Sum of electronic and zero-point Energies= $\quad-381.965784$

Sum of electronic and thermal Energies= $\quad-381.957174$

Sum of electronic and thermal Enthalpies= -381.956230

Sum of electronic and thermal Free Energies= $\quad-381.999582$

$\begin{array}{cc} & \text { KCAL/MOL } \\ \text { TOTAL } & 103.479\end{array}$

E (Thermal)

$\begin{array}{ll}1 & 6 \\ 2 & 6 \\ 3 & 6 \\ 4 & 6 \\ 5 & 7 \\ 6 & 1\end{array}$
6
6
6
6
1

CV

CAL/MOL-KELVIN 32.091
$S$

CAL/MOL-KELVIN 91.241

0.471975
-0.690688
1.778297
1.822718
-2.637765
2.778279

-1.102490
-0.167921
-0.549203
0.862710
0.038533
1.326591

0.684172

$\odot .885155$

0.082784

$-0.295017$

$-0.714132$

$-0.516107$ 


$\begin{array}{rl}7 & 6 \\ 8 & 1 \\ 9 & 7 \\ 10 & 6 \\ 11 & 1 \\ 12 & 1 \\ 13 & 1 \\ 14 & 1 \\ 15 & 6 \\ 16 & 1 \\ 17 & 1 \\ 18 & 1 \\ 19 & 1\end{array}$

$\begin{array}{rr}0.542528 & 1.615318 \\ -1.185039 & -0.352858 \\ -1.955061 & -0.714170 \\ -0.440416 & 1.317185 \\ 0.731403 & 2.693611 \\ 0.054135 & 1.353519 \\ -0.020016 & 1.717299 \\ -1.396593 & 1.825832 \\ 0.898600 & -1.528678 \\ 0.343419 & -1.141818 \\ 1.258628 & -2.546248 \\ 0.588524 & -1.857518 \\ 2.698953 & -0.970468\end{array}$

$-0.482570$

1.841033

$-0.160230$

0.668764

$-0.532115$

$-1.438773$

1. 602044

0.500412

$-0.695095$

$-1.544421$

$-0.819515$

1.457786

๑. 481028 


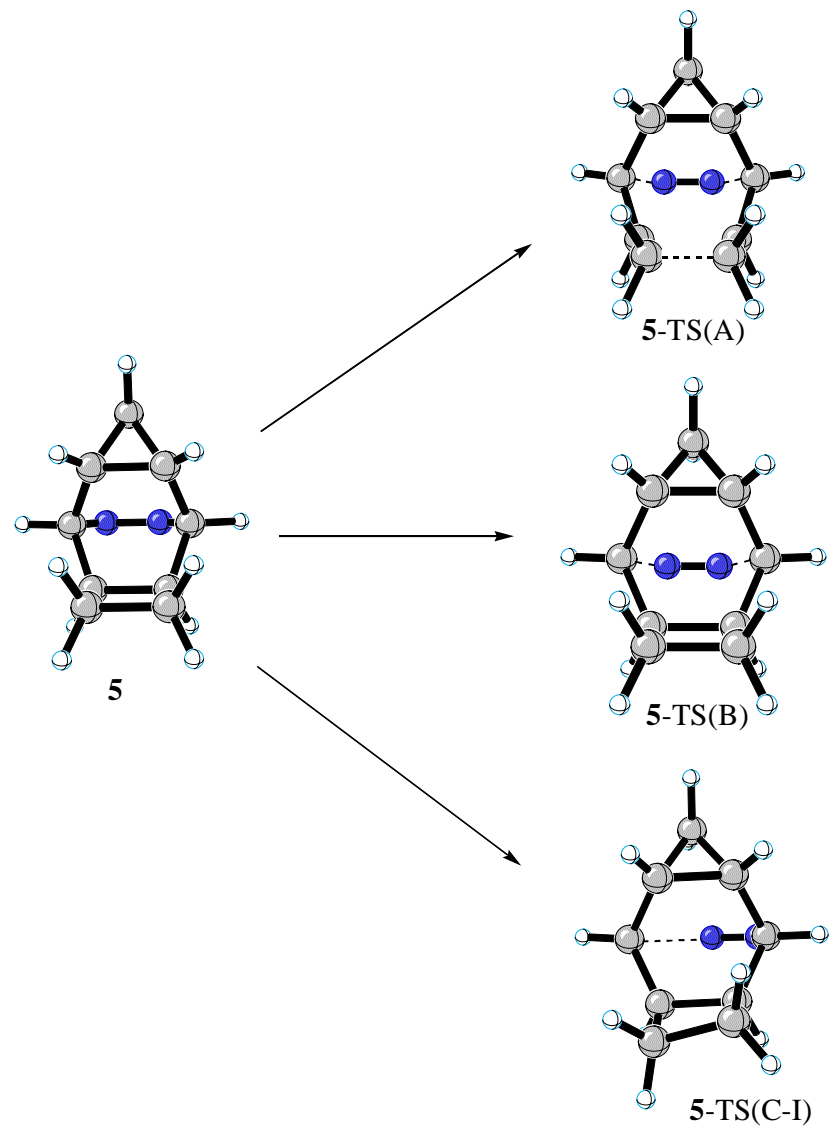

Reactant 5: endo-cyclopropane exo-cyclobutane DBO

RB3LYP/6-31G(d)

$E(R B+H F-L Y P)=-459.566078821$

Sum of electronic and zero-point Energies= -459.366218

Sum of electronic and thermal Energies= $\quad-459.358545$

Sum of electronic and thermal Enthalpies= -459.357601

Sum of electronic and thermal Free Energies= $\quad-459.398374$ $\begin{array}{cr}\mathrm{KCAL} / \mathrm{MOL} \\ \text { TOTAL } & 130.229\end{array}$
CV $S$
E (Thermal)
CAL/MOL-KELVIN CAL/MOL-KELVIN

$33.567 \quad 85.814$

$\begin{array}{rl}1 & 6 \\ 2 & 6 \\ 3 & 1 \\ 4 & 6 \\ 5 & 1 \\ 6 & 6 \\ 7 & 6 \\ 8 & 6 \\ 9 & 1 \\ 10 & 7 \\ 11 & 6 \\ 12 & 1 \\ 13 & 7\end{array}$

-.338386
-.614711
1.886836
-.614711
-1.542178
.538769
.538769
-.338386
-1.542178
-1.425269
1.023099
-.443669
-1.425269

$-.365534$

1. 083052

1.263312

.780164

$-.492698$

1. 083052

$-1.311376$

1.440883

$-.780164$

2.131530

1.236486

2.131530

$-.365534$

.778070

$-.778070$

$-1.263312$

$1.440883-1.236486$

$-1.174500 \quad-.625443$

$-.844568 \quad .753760$

$-.476904 \quad-2.345372$

$-1.174500 \quad .625443$ 


$\begin{array}{rrrrr}14 & 1 & -.443669 & -.476904 & 2.345372 \\ 15 & 6 & 1.023099 & -.844568 & -.753760 \\ 16 & 1 & 1.886836 & -.492698 & 1.311376 \\ 17 & 1 & 2.139427 & -2.599982 & .000000 \\ 18 & 6 & 1.152298 & -2.146628 & .00000 \odot \\ 19 & 1 & .255386 & 3.087526 & -1.228265 \\ 20 & 1 & 1.473896 & 1.805228 & 1.241234 \\ 21 & 1 & .255386 & 3.087526 & 1.228265 \\ 22 & 1 & 1.473896 & 1.805228 & -1.241234 \\ 23 & 1 & .334757 & -2.859093 & .000000\end{array}$

CASPT2 $(12,12) / 6-31 G * / / B 3 L Y P / 6-31 G^{*}$

Reference energy: $\quad-456.7097543556$

Total energy: $\quad-458.0779905631$

Transition structure 5-TS(A): pericyclic loss of nitrogen

RB3LYP/6-31G(d)

$E(R B+H F-L Y P)=-459.494372926$

Sum of electronic and zero-point Energies= $\quad-459.301170$

Sum of electronic and thermal Energies= -459.292073

Sum of electronic and thermal Enthalpies= $\quad-459.291129$

Sum of electronic and thermal Free Energies= $\quad-459.334737$

$\begin{array}{cccc} & \text { KCAL/MOL } & \text { CAL/MOL }- \text { KELVIN } & \text { CAL/MOL-KELVIN } \\ \text { TOTAL } & 126.945 & 37.370 & 91.781\end{array}$

$\begin{array}{rrrrr}1 & 6 & 1.000038 & -0.645810 & 0.997116 \\ 2 & 6 & -0.269033 & -0.139804 & 1.406527 \\ 3 & 6 & 1.000117 & -0.646098 & -0.996909 \\ 4 & 6 & -0.268840 & -0.139939 & -1.406463 \\ 5 & 7 & -1.509772 & -1.532608 & 0.575392 \\ 6 & 6 & -0.674304 & 1.161077 & 0.765799 \\ 7 & 1 & -0.714949 & -0.431520 & 2.349567 \\ 8 & 1 & -0.714772 & -0.431760 & -2.349465 \\ 9 & 6 & -0.674066 & 1.161046 & -0.765944 \\ 10 & 7 & -1.509759 & -1.532644 & -0.575221 \\ 11 & 1 & -0.266567 & 2.052708 & -1.244450 \\ 12 & 6 & 2.203815 & 0.291547 & 0.766282 \\ 13 & 1 & 3.112833 & -0.130099 & 1.210002 \\ 14 & 1 & 1.257307 & -1.617112 & 1.426706 \\ 15 & 1 & 1.257182 & -1.617532 & -1.426324 \\ 16 & 1 & 3.113098 & -0.131204 & -1.209347 \\ 17 & 1 & 2.064034 & 1.282747 & 1.210418 \\ 18 & 6 & 2.204071 & 0.291083 & -0.766263 \\ 19 & 1 & 2.064753 & 1.282032 & -1.211117 \\ 20 & 6 & -1.967262 & 1.364871 & -0.00 \odot 275 \\ 21 & 1 & -0.267024 & 2.052808 & 1.244357 \\ 22 & 1 & -2.719589 & 0.586352 & -0.000445 \\ 23 & 1 & -2.376807 & 2.371518 & -0.000320\end{array}$

CASPT2 $(12,12) / 6-31 G * / / B 3 L Y P / 6-31 G^{*}$

Reference energy: $\quad-456.6438742464$

Total energy: $\quad-458.0023795694$

S 39 endo-cyclopropane-exo-cyclobutane DBO, $\mathbf{5}$ 
Transition structure 5-TS(B): 2-bond CN cleavage

\begin{tabular}{|c|c|c|c|c|c|c|}
\hline \multicolumn{7}{|c|}{ UB3LYP/6 - 31G(d) } \\
\hline $\mathrm{E}(\mathrm{UB}+\mathrm{H}$ & $F-L Y P)=-4$ & 459.500787374 & & & & \\
\hline Sum of & electronic & and zero-point & hergies= & & -459 & 308946 \\
\hline Sum of & electronic & and thermal En & gies= & & -459 & 299673 \\
\hline Sum of & electronic & and thermal En & alpies= & & -459 & 298729 \\
\hline Sum of & electronic & and thermal $\mathrm{Fr}$ & Energies= & & -459 & 343312 \\
\hline & & E (Thermal) & & & & $S$ \\
\hline & & KCAL/MOL & $\mathrm{CAL} / \mathrm{MOL}$ & ELVIN & & /MOL - KELVIN \\
\hline TOTAL & & 126.201 & & 524 & & 93.833 \\
\hline 1 & 6 & & -0.087563 & -0.24 & 999 & 1.381049 \\
\hline 2 & 7 & & -1.602338 & -1.50 & 280 & 0.573211 \\
\hline 3 & 7 & & -1.602338 & -1.50 & 280 & -0.573211 \\
\hline 4 & 6 & & $-\odot .087563$ & -0.24 & 999 & -1.381049 \\
\hline 5 & 1 & & -0.284849 & -0.47 & 466 & -2.424299 \\
\hline 6 & 1 & & 2.096137 & -0.42 & 5578 & -1.270801 \\
\hline 7 & 6 & & -0.635898 & 1.01 & 1003 & $-\odot .802985$ \\
\hline 8 & 6 & & 1.182617 & -0.74 & 238 & $\odot .762013$ \\
\hline 9 & 1 & & -0.284849 & -0.47 & 466 & 2.424299 \\
\hline 10 & 6 & & 1.182617 & -0.74 & 238 & -0.762013 \\
\hline 11 & 6 & & 1.296950 & -2.05 & 1980 & $\odot . \odot \odot \odot \odot \odot \odot$ \\
\hline 12 & 1 & & 2.096137 & -0.42 & 3578 & 1.270801 \\
\hline 13 & 1 & & 2.278019 & -2.51 & 3829 & $\odot .000000$ \\
\hline 14 & 1 & & -1.618056 & 1.23 & 1080 & -1.232204 \\
\hline 15 & 6 & & -0.635898 & 1.01 & 4003 & 0.802985 \\
\hline 16 & 1 & & -1.618056 & 1.23 & 7080 & 1.232204 \\
\hline 17 & 1 & & 0.473140 & -2.75 & 3379 & $\odot . \odot \odot \odot \odot \odot \odot$ \\
\hline 18 & 6 & & $\odot .307149$ & 2.26 & 4756 & $-\odot .776095$ \\
\hline 19 & 6 & & 0.307149 & 2.26 & 4756 & 0.776095 \\
\hline 20 & 1 & & -0.131002 & 3.15 & 3960 & -1.240897 \\
\hline 21 & 1 & & 1.289881 & 2.09 & 7876 & -1.227381 \\
\hline 22 & 1 & & -0.131002 & 3.15 & 3960 & 1.240897 \\
\hline 23 & 1 & & 1.289881 & 2.09 & 7876 & 1.227381 \\
\hline
\end{tabular}

CASPT2 $(12,12) / 6-31 G * / / U B 3 L Y P / 6-31 G *$

Reference energy: $\quad-456.6624339882$

Total energy: $\quad-458.0123237339$

Transition structure 5-TS(C-I): 1-bond CN cleavage

UB3LYP/6-31G(d)

$E(U B+H F-L Y P)=-459.506253896$

Sum of electronic and zero-point Energies= -459.312754

Sum of electronic and thermal Energies= $\quad-459.303877$

Sum of electronic and thermal Enthalpies= -459.302933

Sum of electronic and thermal Free Energies= $\quad-459.346281$

E (Thermal)
KCAL/MOL

CV

$S$

TOTAL

126.993

$\begin{array}{cr}\text { CAL/MOL-KELVIN } & \text { CAL/MOL-KELVIN } \\ 36.555 & 91.234\end{array}$ 


\begin{tabular}{|c|c|c|c|c|}
\hline 1 & 6 & -0.494712 & -0.835018 & -0.918046 \\
\hline 2 & 7 & -1.413146 & -1.668377 & $-\odot .003406$ \\
\hline 3 & 7 & -1.594900 & -1.368973 & 1.124213 \\
\hline 4 & 6 & 0.064429 & 0.918483 & 1.228568 \\
\hline 5 & 1 & $-\odot .055875$ & 1.341995 & 2.222944 \\
\hline 6 & 1 & $-\odot .286879$ & 2.569619 & $-\odot .187700$ \\
\hline 7 & 6 & 1.128271 & -0.101913 & $\odot .996670$ \\
\hline 8 & 6 & $-\odot .958035$ & 0.616704 & -1.065161 \\
\hline 9 & 1 & -0.543338 & -1.343954 & -1.883886 \\
\hline 10 & 6 & $-\odot .612455$ & 1.558745 & $\odot .076445$ \\
\hline 11 & 6 & -2.061044 & 1.247684 & $-\odot .251180$ \\
\hline 12 & 1 & -0.850252 & 0.991923 & -2.080190 \\
\hline 13 & 1 & -2.639002 & 2.023968 & $-\odot .745960$ \\
\hline 14 & 1 & 1.274304 & -0.703219 & 1.899314 \\
\hline 15 & 6 & 0.911149 & -0.935841 & -0.317394 \\
\hline 16 & 1 & 1.170315 & -1.990223 & -0.173792 \\
\hline 17 & 1 & -2.623913 & 0.641047 & 0.448258 \\
\hline 18 & 6 & 2.061596 & -0.152179 & -1.008788 \\
\hline 19 & 6 & 2.479500 & 0.423702 & $\odot .370085$ \\
\hline 20 & 1 & 2.644868 & 1.504421 & $\odot .419035$ \\
\hline 21 & 1 & 3.351183 & $-\odot .081285$ & $\odot .799094$ \\
\hline 22 & 1 & 1.698731 & 0.622215 & -1.691314 \\
\hline 23 & 1 & 2.803994 & -0.757267 & -1.538641 \\
\hline \multicolumn{5}{|c|}{ CASPT2 $(12,12) / 6-31 G^{*} / / U B 3 L Y P / 6-31 G^{*}$} \\
\hline & energy: & \multicolumn{3}{|c|}{-456.6539345193} \\
\hline & rgy: & \multicolumn{3}{|c|}{-458.0169807132} \\
\hline
\end{tabular}



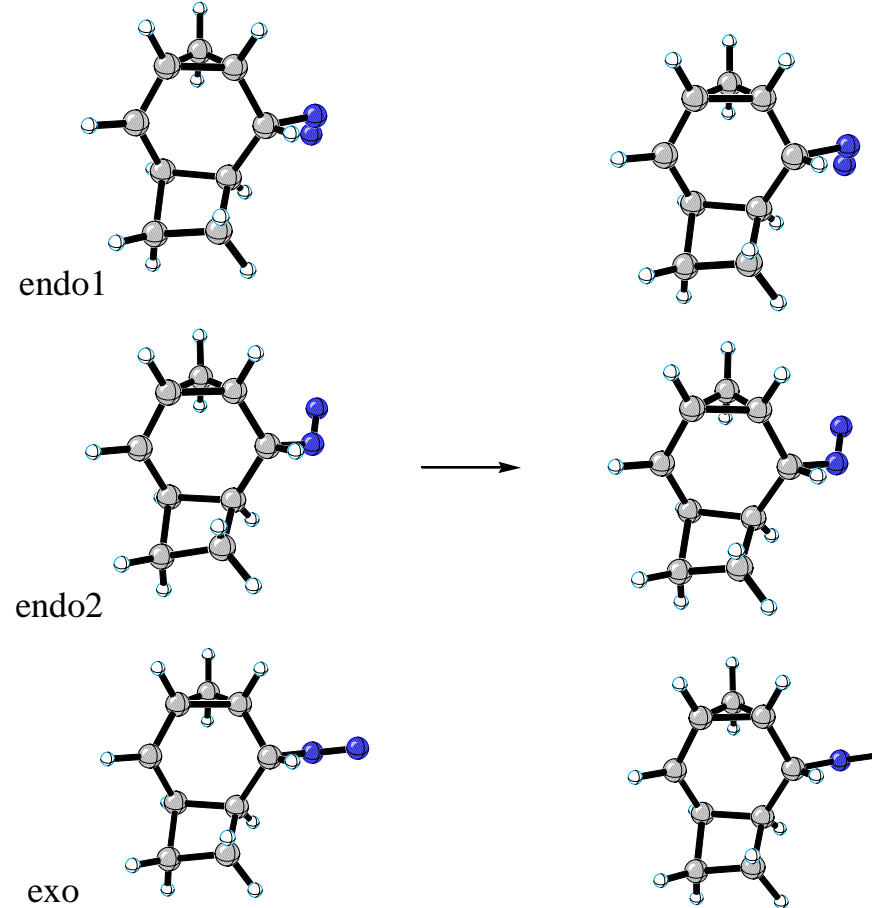

diazenyl diradical

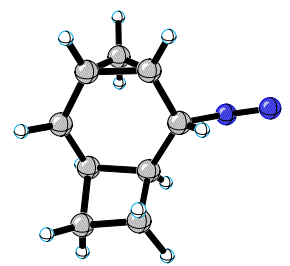

5-TS(C-II)

Intermediate from 5: diazenyl dirad, endo1 $\mathrm{N}=\mathrm{N}$

UB3LYP/6 - 31G(d)

$E(U B+H F-L Y P)=-459.512184637$

Sum of electronic and zero-point Energies $=\quad-459.319809$

Sum of electronic and thermal Energies= $\quad$-459.309509

Sum of electronic and thermal Enthalpies $=\quad-459.308565$

Sum of electronic and thermal Free Energies $=\quad-459.356065$

TOTAL

E (Thermal) KCAL/MOL 127.181
CV CAL/MOL-KELVIN 39.626
$S$

CAL/MOL - KELVIN 99.972

$\begin{array}{rl}1 & 6 \\ 2 & 6 \\ 3 & 1 \\ 4 & 6 \\ 5 & 6 \\ 6 & 6 \\ 7 & 1 \\ 8 & 6 \\ 9 & 1 \\ 10 & 1 \\ 11 & 6 \\ 12 & 1 \\ 13 & 6 \\ 14 & 6 \\ 15 & 1\end{array}$

0.808573
-0.436148
0.168509
-1.363822
1.318716
-1.210364
1.118659
1.190364
-2.065736
2.097340
0.121896
1.876730
-1.668779
-2.594351
-1.265100

$-0.493519$

$-0.892207$

2. 947812

0.222379

1.724915

1.544420

1.198913

$\odot .968408$

2. 205291

2.479278

2.013868

1. 254367

$-1.413477$

$-0.616113$

0.313421
๑. 691916

$-0.107359$

0.860103

$-0.726661$

$-0.609034$

$-0.063706$

$-1.538540$

0.677776

0.043778

$-0.680467$

๑. 305361

1. 471065

๑. 682598

$-0.271525$

$-1.822055$ 


$\begin{array}{rrrrr}16 & 1 & -0.150300 & -1.604703 & -0.886959 \\ 17 & 1 & -3.423084 & -0.064246 & 0.183471 \\ 18 & 1 & -2.988309 & -1.236477 & -1.082946 \\ 19 & 1 & -1.694847 & -1.006506 & 1.699954 \\ 20 & 1 & -1.803761 & -2.498558 & 0.737127 \\ 21 & 1 & 0.718053 & -0.829063 & 1.729362 \\ 22 & 7 & 2.127880 & -1.273364 & 0.221209 \\ 23 & 7 & 2.217167 & -1.799717 & -0.819794\end{array}$

CASPT2 $(12,12) / 6-31 G * / / U B 3 L Y P / 6-31 G$ *

Reference energy: $\quad-456.6581713350$

Total energy: $\quad-458.0162624661$

Intermediate from 5: diazenyl dirad, endo2 $\mathrm{N}=\mathrm{N}$

UB3LYP/6-31G(d)

$E(U B+H F-L Y P)=-459.5117236$

Sum of electronic and zero-point Energies= $\quad-459.319364$

Sum of electronic and thermal Energies= $\quad-459.309122$

Sum of electronic and thermal Enthalpies= $\quad-459.308178$

Sum of electronic and thermal Free Energies $=\quad-459.355265$

$\begin{array}{cccc} & \text { E (Thermal }) & \text { CV } & \text { S } \\ \text { KCAL/MOL } & \text { CAL/MOL-KELVIN } & \text { CAL/MOL-KELVIN } \\ \text { TOTAL } & 127.134 & 39.609 & 99.104\end{array}$

$\begin{array}{rrrrr}1 & 6 & 0.734540 & -0.669664 & 0.583273 \\ 2 & 6 & -0.579150 & -0.930693 & -0.164751 \\ 3 & 1 & 0.259347 & 2.777660 & 1.045133 \\ 4 & 6 & -1.405031 & 0.269301 & -0.760214 \\ 5 & 6 & 1.387742 & 1.619431 & -0.491547 \\ 6 & 6 & -1.164320 & 1.551138 & -0.041114 \\ 7 & 1 & 1.185218 & 1.192571 & -1.469630 \\ 8 & 6 & 1.174702 & 0.762657 & 0.717355 \\ 9 & 1 & -1.968553 & 2.275618 & 0.052772 \\ 10 & 1 & 2.211457 & 2.327274 & -0.474297 \\ 11 & 6 & 0.177156 & 1.898692 & 0.410325 \\ 12 & 1 & 1.852346 & 0.946147 & 1.547816 \\ 13 & 6 & -1.838883 & -1.323140 & 0.655828 \\ 14 & 6 & -2.698890 & -0.477899 & -0.317945 \\ 15 & 1 & -1.298949 & 0.392066 & -1.851714 \\ 16 & 1 & -0.403663 & -1.679096 & -0.944401 \\ 17 & 1 & -3.497261 & 0.132796 & 0.115547 \\ 18 & 1 & -3.116856 & -1.082904 & -1.129336 \\ 19 & 1 & -1.816546 & -0.878322 & 1.657035 \\ 20 & 1 & -2.062945 & -2.390644 & 0.748995 \\ 21 & 1 & 0.687681 & -1.144975 & 1.568398 \\ 22 & 7 & 1.861772 & -1.572179 & -0.128429 \\ 23 & 7 & 2.887018 & -1.151696 & -0.502082\end{array}$

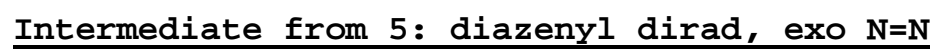

UB3LYP/6-31G(d)

$E(U B+H F-L Y P)=-459.512901111$

Sum of electronic and zero-point Energies=

Sum of electronic and thermal Energies=

$-459.320440$

$-459.310246$ 
Sum of electronic and thermal Enthalpies=

Sum of electronic and thermal Free Energies=

E (Thermal) $\mathrm{KCAL} / \mathrm{MOL}$

TOTAL 127.168
$-459.309302$

$-459.356447$

$\begin{array}{rl}1 & 6 \\ 2 & 6 \\ 3 & 1 \\ 4 & 6 \\ 5 & 6 \\ 6 & 6 \\ 7 & 1 \\ 8 & 6 \\ 9 & 1 \\ 10 & 1 \\ 11 & 6 \\ 12 & 1 \\ 13 & 6 \\ 14 & 6 \\ 15 & 1 \\ 16 & 1 \\ 17 & 1 \\ 18 & 1 \\ 19 & 1 \\ 20 & 1 \\ 21 & 1 \\ 22 & 7 \\ 23 & 7\end{array}$

CV

CAL/MOL-KELVIN 39.522
$S$ CAL/MOL-KELVIN 99.225

$\begin{array}{rrr}-0.908984 & -0.408278 & -0.395082 \\ 0.365619 & -0.908421 & 0.298532 \\ 0.211809 & 2.830316 & -1.125313 \\ 1.529571 & 0.101957 & 0.637092 \\ -0.885307 & 2.000887 & 0.630899 \\ 1.483327 & 1.337215 & -0.189347 \\ -0.635233 & 1.562966 & 1.592857 \\ -1.058176 & 1.084422 & -0.541104 \\ 2.402099 & 1.801682 & -0.535205 \\ -1.524098 & 2.877704 & 0.690124 \\ 0.198905 & 1.977019 & -0.451901 \\ -1.798103 & 1.384224 & -1.279633 \\ 1.383291 & -1.724264 & -0.546152 \\ 2.542765 & -0.982260 & 0.166855 \\ 1.597514 & 0.343751 & 1.711700 \\ 0.087596 & -1.461520 & 1.201366 \\ 3.373444 & -0.638722 & -0.457783 \\ 2.947849 & -1.554699 & 1.007478 \\ 1.327111 & -1.456239 & -1.607030 \\ 1.343595 & -2.814695 & -0.458795 \\ -1.024182 & -0.888679 & -1.372654 \\ -2.148361 & -0.904463 & 0.461574 \\ -3.025277 & -1.503503 & -0.036697\end{array}$

Transition structure 5-TS(C-II): cleave 2nd CN, endo1 $\mathrm{N}=\mathrm{N}$

UB3LYP/6-31G(d)
$\mathrm{E}(\mathrm{UB}+\mathrm{HF}-\mathrm{LYP})=-459.512102737$
Sum of electronic and zero-point Energies=
Sum of electronic and thermal Energies=
Sum of electronic and thermal Enthalpies=
Sum of electronic and thermal Free Energies=

E (Thermal)

CV

CAL/MOL-KELVIN CAL/MOL-KELVIN

126.327
38.215

98.869

$\begin{array}{rrr}0.799486 & -0.460801 & 0.706970 \\ -0.417388 & -0.892336 & -0.106731 \\ 0.112133 & 2.964527 & 0.845141 \\ -1.374605 & 0.205020 & -0.720178 \\ 1.273128 & 1.745685 & -0.616366 \\ -1.248094 & 1.528720 & -0.055645 \\ 1.079710 & 1.208711 & -1.540950 \\ 1.168538 & 0.994905 & 0.675868 \\ -2.124782 & 2.145866 & 0.116980 \\ 2.039529 & 2.511585 & -0.696684 \\ 0.076326 & 2.027525 & 0.294946 \\ 1.850757 & 1.301639 & 1.464875 \\ -1.640594 & -1.462650 & 0.664677\end{array}$




$\begin{array}{rrrrr}14 & 6 & -2.584294 & -0.665759 & -0.272713 \\ 15 & 1 & -1.273947 & 0.299913 & -1.814836 \\ 16 & 1 & -0.101720 & -1.582664 & -0.895405 \\ 17 & 1 & -3.419958 & -0.137204 & 0.197192 \\ 18 & 1 & -2.973137 & -1.278784 & -1.092035 \\ 19 & 1 & -1.680060 & -1.083945 & 1.692397 \\ 20 & 1 & -1.748991 & -2.551723 & 0.690232 \\ 21 & 1 & 0.726443 & -0.806310 & 1.741371 \\ 22 & 7 & 2.185116 & -1.258426 & 0.228264 \\ 23 & 7 & 2.271884 & -1.757784 & -0.818728\end{array}$

CASPT2 $(12,12) / 6-31 G * / / U B 3 L Y P / 6-31 G$ *

Reference energy: $\quad-456.6598074149$

Total energy: $\quad-458.0157648791$

Transition structure 5-TS(C-II): cleave 2nd $\mathrm{CN}$, endo2 $\mathrm{N}=\mathrm{N}$

UB3LYP/6-31G(d)

$E(U B+H F-L Y P)=-459.511682164$

Sum of electronic and zero-point Energies= $\quad-459.320157$

Sum of electronic and thermal Energies= $\quad-459.310335$

Sum of electronic and thermal Enthalpies= -459.309391

Sum of electronic and thermal Free Energies= $\quad-459.355766$

$\begin{array}{cccc} & \text { KCAL/MOL } & \text { CAL/MOL-KELVIN } & \text { CAL/MOL-KELVIN } \\ \text { TOTAL } & 126.347 & 38.082 & 97.606\end{array}$

$\begin{array}{rrrrr}1 & 6 & 0.721596 & -0.653883 & 0.613602 \\ 2 & 6 & -0.574341 & -0.932464 & -0.148902 \\ 3 & 1 & 0.238650 & 2.793921 & 1.021971 \\ 4 & 6 & -1.399184 & 0.258229 & -0.767845 \\ 5 & 6 & 1.384510 & 1.614805 & -0.483458 \\ 6 & 6 & -1.171406 & 1.548596 & -0.059455 \\ 7 & 1 & 1.195843 & 1.173028 & -1.457593 \\ 8 & 6 & 1.162627 & 0.771850 & 0.733909 \\ 9 & 1 & -1.988825 & 2.254878 & 0.054800 \\ 10 & 1 & 2.206906 & 2.324149 & -0.465937 \\ 11 & 6 & 0.163772 & 1.908139 & 0.395897 \\ 12 & 1 & 1.828411 & 0.969948 & 1.570473 \\ 13 & 6 & -1.843475 & -1.323434 & 0.658916 \\ 14 & 6 & -2.694410 & -0.489893 & -0.333121 \\ 15 & 1 & -1.278789 & 0.368780 & -1.859045 \\ 16 & 1 & -0.385806 & -1.688015 & -0.918881 \\ 17 & 1 & -3.499739 & 0.121883 & 0.085923 \\ 18 & 1 & -3.101698 & -1.103739 & -1.143309 \\ 19 & 1 & -1.836109 & -0.870751 & 1.656784 \\ 20 & 1 & -2.064231 & -2.391120 & 0.757538 \\ 21 & 1 & 0.690328 & -1.138363 & 1.593591 \\ 22 & 7 & 1.896782 & -1.582032 & -0.118524 \\ 23 & 7 & 2.888493 & -1.136006 & -0.531986\end{array}$

Transition structure 5-TS(C-II): cleave 2nd CN, exo $\mathrm{N}=\mathrm{N}$

UB3LYP/6-31G(d)

$E(U B+H F-L Y P)=-459.5126225$ 
Sum of electronic and zero-point Energies=

Sum of electronic and thermal Energies=

Sum of electronic and thermal Enthalpies=

Sum of electronic and thermal Free Energies=

$$
\begin{array}{r}
-459.321467 \\
-459.311516 \\
-459.310571 \\
-459.357305
\end{array}
$$

CV

CAL/MOL-KELVIN CAL/MOL-KELVIN 38.399
98.359
E (Thermal) $\mathrm{KCAL} / \mathrm{MOL}$ 126.196

\begin{abstract}
$-0.888256$
$\odot .352415$

$\odot .274933$

1.538823

$-0.836406$

1.521064

$-0.600946$

$-1.031603$

2. 428594

$-1.462838$

0.253270

$-1.758100$

1. 364081

2. 531633

1.593854

๑. 050814

3. 370206

2. 925487

1. 322655

1. 303865

$-1.040355$

$-2.218841$

$-3.100916$
\end{abstract}
$-0.378476$
$-0.909136$
2. 847120
$\odot .080991$
2. 001738
1. 318465
1.544710
1.102589
1. 685191
2. 886617
1.988855
1.429862
$-1.753693$
$-1.024543$
0.319234
$-1.446709$
$-0.699764$
$-1.598066$
$-1.500260$
$-2.841899$
$-0.868214$
$-0.897263$
$-1.433959$

$-0.423853$

$\odot .287693$

$-1.097058$

0.636503

0.637407

$-0.187419$

1.594284

$-0.544349$

$-0.657249$

0.707944

$-0.430398$

$-1.284131$

$-0.536296$

0.178020

1.711936

1.192678

$-0.446422$

1.023156

$-1.601510$

$-0.434176$

$-1.389670$

0.479260

$-0.054064$ 


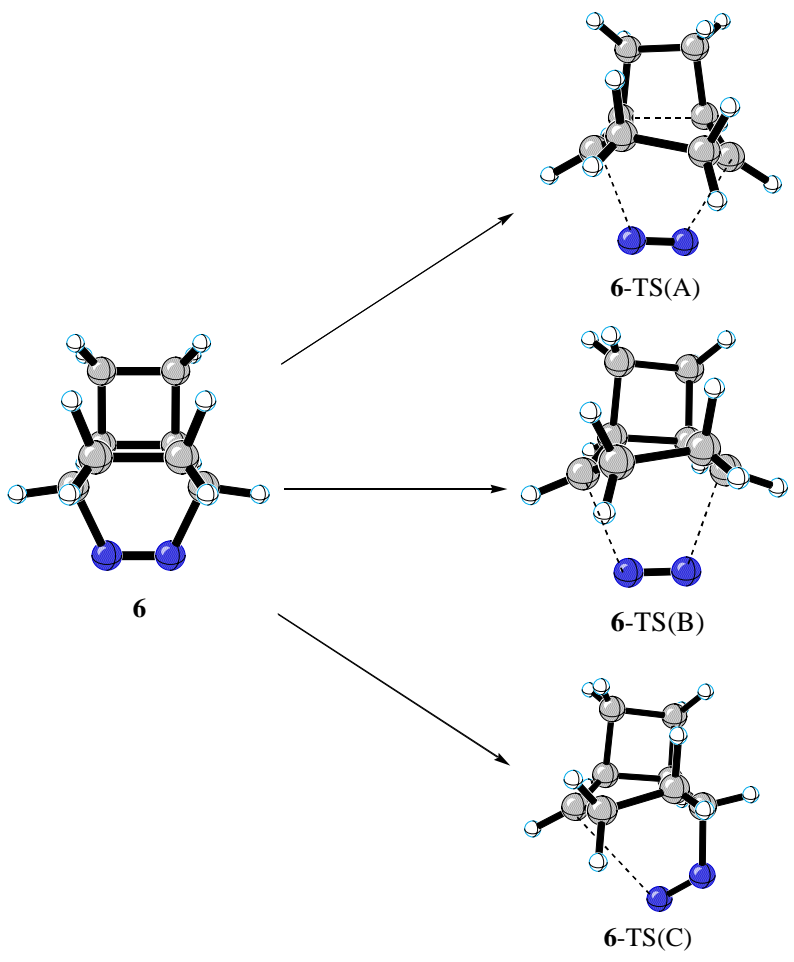

Reactant 6: exo-cyclobutane DBO

RB3LYP/6-31G(d)

$E(R B+H F-L Y P)=-421.499202913$

Sum of electronic and zero-point Energies= -421.304799

Sum of electronic and thermal Energies= $\quad-421.297336$

Sum of electronic and thermal Enthalpies= $\quad-421.296392$

Sum of electronic and thermal Free Energies= $\quad-421.337408$

E (Thermal)
KCAL/MOL

CV

$S$

TOTAL

126.673

CAL/MOL-KELVIN CAL/MOL-KELVIN

$31.264 \quad 86.326$

$\begin{array}{rl}1 & 6 \\ 2 & 6 \\ 3 & 1 \\ 4 & 6 \\ 5 & 1 \\ 6 & 6 \\ 7 & 6 \\ 8 & 6 \\ 9 & 1 \\ 10 & 7 \\ 11 & 6 \\ 12 & 1 \\ 13 & 7 \\ 14 & 1 \\ 15 & 6 \\ 16 & 1\end{array}$

-.590066
.741921
-.017085
.741930
.859921
2.047371
2.047393
-.590042
.859915
-1.666170
-.797005
-.716792
-1.666179
-.716835
-.796973
-.017148

1.259431

$-.103618$

$.778515-.716777$

$-1.183036$

$-.778544$

1.996621

1. 235128

.778987

$-.778962$

$-1.259442$

$-1.235205$

$-.625471$

$-.716741$

$-1.703966$

.134651

.134666

$-.103536$

$-1.703910$

.773955

$-.932572$

$-2.341812$

1. 347169

.625385

$-.183887$

2.341793

$-.773874$

$-.932617$

- . 184042

1.183193

1. 347221

1.996558 


$\begin{array}{rrrrr}17 & 1 & -1.750648 & 1.167725 & 1.712670 \\ 18 & 1 & -1.750593 & -1.167658 & 1.712770 \\ 19 & 1 & 2.900703 & 1.226871 & -.382865 \\ 20 & 1 & 1.973523 & 1.246904 & 1.120507 \\ 21 & 1 & 2.900727 & -1.226827 & -.382864 \\ 22 & 1 & 1.973583 & -1.246869 & 1.120531\end{array}$

CASPT2 $(12,12) / 6-31 G * / / B 3 L Y P / 6-31 G *$

Reference energy: $\quad-418.8791480819$

Total energy: $\quad-420.1172644763$

\section{Transition structure 6-TS(A): pericyclic loss of nitrogen}

\begin{tabular}{|c|c|c|c|c|c|c|}
\hline \multicolumn{7}{|c|}{ RB3LYP/6-31G(d) } \\
\hline$E(R B+H$ & $=-$ LYP $)=-$ & 421.432387621 & & & & \\
\hline Sum of & electronic & and zero-point & nergies= & & $-42\rfloor$ & 245022 \\
\hline Sum of & electronic & and thermal Ene & gies $=$ & & -421 & 236143 \\
\hline Sum of & electronic & and thermal Ent & alpies= & & -421 & 235198 \\
\hline Sum of & electronic & and thermal Fre & Energies= & & -421 & 278159 \\
\hline & & E (Thermal) & & & & $\mathrm{S}$ \\
\hline & & $\mathrm{KCAL} / \mathrm{MOL}$ & $\mathrm{CAL} / \mathrm{MOL}$ & ELVIN & & MOL-KELVIN \\
\hline TOTAL & & 123.146 & & 459 & & 90.417 \\
\hline 1 & 6 & & -.657028 & .72 & 3982 & -.925887 \\
\hline 2 & 1 & & 1.157919 & -.57 & 6757 & 2.372647 \\
\hline 3 & 1 & & 1.794144 & 1.45 & 6369 & 1.317965 \\
\hline 4 & 6 & & .503319 & 1.35 & 7442 & -.375700 \\
\hline 5 & 6 & & -.715449 & -1.07 & 3323 & -.462162 \\
\hline 6 & 6 & & .279527 & -1.29 & 7464 & .550472 \\
\hline 7 & 7 & & 2. $09 \odot 992$ & .11 & 1758 & -1.043414 \\
\hline 8 & 6 & & .752483 & 1.20 & 9441 & 1.093451 \\
\hline 9 & 1 & & 1.052859 & 2.10 & 6405 & -.927351 \\
\hline 10 & 1 & & .744415 & -2.26 & 5457 & .665850 \\
\hline 11 & 6 & & .405172 & -.27 & 8131 & 1.637149 \\
\hline 12 & 7 & & 2.031077 & -.95 & อ271 & -.624273 \\
\hline 13 & 1 & & -.547613 & -.19 & 2206 & 2.177445 \\
\hline 14 & 1 & & .131837 & 1.88 & 9363 & 1.679189 \\
\hline 15 & 6 & & -2.012931 & .78 & 3153 & -.181780 \\
\hline 16 & 1 & & -2.792713 & 1.31 & 8130 & -.736680 \\
\hline 17 & 1 & & -.756374 & .83 & 5662 & -2.008163 \\
\hline 18 & 1 & & -.625769 & -1.76 & 7550 & -1.303100 \\
\hline 19 & 1 & & -2.863354 & -1.14 & 1655 & -.826909 \\
\hline 20 & 1 & & -1.922291 & 1.26 & 8636 & .794445 \\
\hline 21 & 6 & & -2.176846 & -.73 & 8812 & - . .074236 \\
\hline 22 & 1 & & -2.497030 & -1.12 & 5073 & .900633 \\
\hline
\end{tabular}

CASPT2 $(12,12) / 6-31 G * / / B 3 L Y P / 6-31 G^{*}$

Reference energy: $\quad-418.8245695265$

Total energy: $\quad-420.0496628362$

Transition structure 4-TS(B): 2-bond CN cleavage

UB3LYP6/-31G(d) 
$E(U B+H F-L Y P)=-421.435593970$

Sum of electronic and zero-point Energies= $\quad-421.249447$

Sum of electronic and thermal Energies= $\quad-421.240446$

Sum of electronic and thermal Enthalpies= $\quad-421.239502$

Sum of electronic and thermal Free Energies= $\quad-421.283032$

$\mathrm{KCAL} / \mathrm{MOL}$
TOTAL
122.457

CV $S$

\begin{tabular}{rrrrr} 
TOTAL & & \multicolumn{2}{c}{35.529} & 91.617 \\
1 & 6 & -0.294666 & -1.187465 & 0.697362 \\
2 & 6 & -0.487355 & -0.052651 & 1.660628 \\
3 & 6 & -0.805708 & 1.319746 & 0.942520 \\
4 & 1 & -1.274795 & -0.289609 & 2.383382 \\
5 & 1 & 0.434410 & 0.081198 & 2.250169 \\
6 & 6 & -0.478643 & 1.295271 & -0.523551 \\
7 & 1 & -1.861851 & 1.587075 & 1.070783 \\
8 & 7 & -2.084223 & -0.039297 & -1.104679 \\
9 & 6 & 0.714897 & -1.005218 & -0.375746 \\
10 & 1 & -0.868574 & 2.087148 & -1.152963 \\
11 & 7 & -2.038965 & -1.030761 & -0.537788 \\
12 & 1 & -0.555080 & -2.191946 & 1.011893 \\
13 & 6 & 0.690511 & 0.501516 & -0.963133 \\
14 & 1 & -0.235547 & 2.108602 & 1.455103 \\
15 & 6 & 2.196098 & -0.761433 & 0.066049 \\
16 & 6 & 2.072515 & 0.747152 & -0.266686 \\
17 & 1 & 0.610950 & -1.789953 & -1.133019 \\
18 & 1 & 0.786211 & 0.508452 & -2.053094 \\
19 & 1 & 2.435424 & -1.010969 & 1.105142 \\
20 & 1 & 2.903226 & -1.292997 & -0.579161 \\
21 & 1 & 1.992142 & 1.378150 & 0.623792 \\
22 & 1 & 2.849904 & 1.173739 & -0.909414
\end{tabular}

CASPT2 $(12,12) / 6-31 G * / / U B 3 L Y P / 6-31 G^{*}$

Reference energy: $\quad-418.8347272285$

Total energy: $\quad-420.0545165019$

Transition structure 4-TS(C-I): 1-bond cleavage of CN

UB3LYP/6-31G(d)

$\mathrm{E}(\mathrm{UB}+\mathrm{HF}-\mathrm{LYP})=-421.437080041$

Sum of electronic and zero-point Energies= $\quad-421.249341$

Sum of electronic and thermal Energies= $\quad-421.240724$

Sum of electronic and thermal Enthalpies= -421.239780

Sum of electronic and thermal Free Energies= $\quad-421.282391$

$\begin{array}{cccc} & \text { KCAL/MOL } & \text { CAL/MOL-KELVIN } & \text { CAL/MOL-KELVIN } \\ \text { TOTAL } & 123.215 & 34.441 & 89.681\end{array}$

$$
\begin{gathered}
\text { E (Thermal) } \\
\text { KCAL/MOL } \\
123.215
\end{gathered}
$$

$\begin{array}{rrr}-0.616089 & -0.925687 & 0.640676 \\ -1.951915 & -1.050019 & -0.129029 \\ -2.127323 & -0.499501 & -1.157782 \\ -0.069587 & 1.479467 & -0.755373 \\ -0.793498 & 1.629103 & 0.550370 \\ 0.241197 & 0.424236 & 2.054247\end{array}$




$\begin{array}{rrrrr}7 & 6 & 0.569495 & -0.986628 & -0.323582 \\ 8 & 1 & -0.614176 & -1.791426 & 1.309550 \\ 9 & 6 & 0.934869 & 0.407795 & -0.959070 \\ 10 & 1 & -1.853303 & 1.841548 & 0.351549 \\ 11 & 1 & -0.430756 & 2.505019 & 1.116455 \\ 12 & 6 & -0.674420 & 0.376843 & 1.451410 \\ 13 & 1 & -1.508804 & 0.337751 & 2.160454 \\ 14 & 6 & 2.251136 & 0.415324 & -0.085580 \\ 15 & 6 & 1.969188 & -1.046420 & 0.352517 \\ 16 & 1 & 2.629725 & -1.780041 & -0.119703 \\ 17 & 1 & 1.977096 & -1.236902 & 1.431871 \\ 18 & 1 & 3.178485 & 0.583962 & -0.641926 \\ 19 & 1 & 2.212889 & 1.135643 & 0.737346 \\ 20 & 1 & 0.386171 & -1.780125 & -1.055696 \\ 21 & 1 & 1.177884 & 0.308671 & -2.021039 \\ 22 & 1 & -0.268312 & 2.199527 & -1.543638\end{array}$

CASPT2 $(12,12) / 6-31 G * / / U B 3 L Y P / 6-31 G$ *

Reference energy: $\quad-418.8206670540$

Total energy:

$-420.0539822558$ 

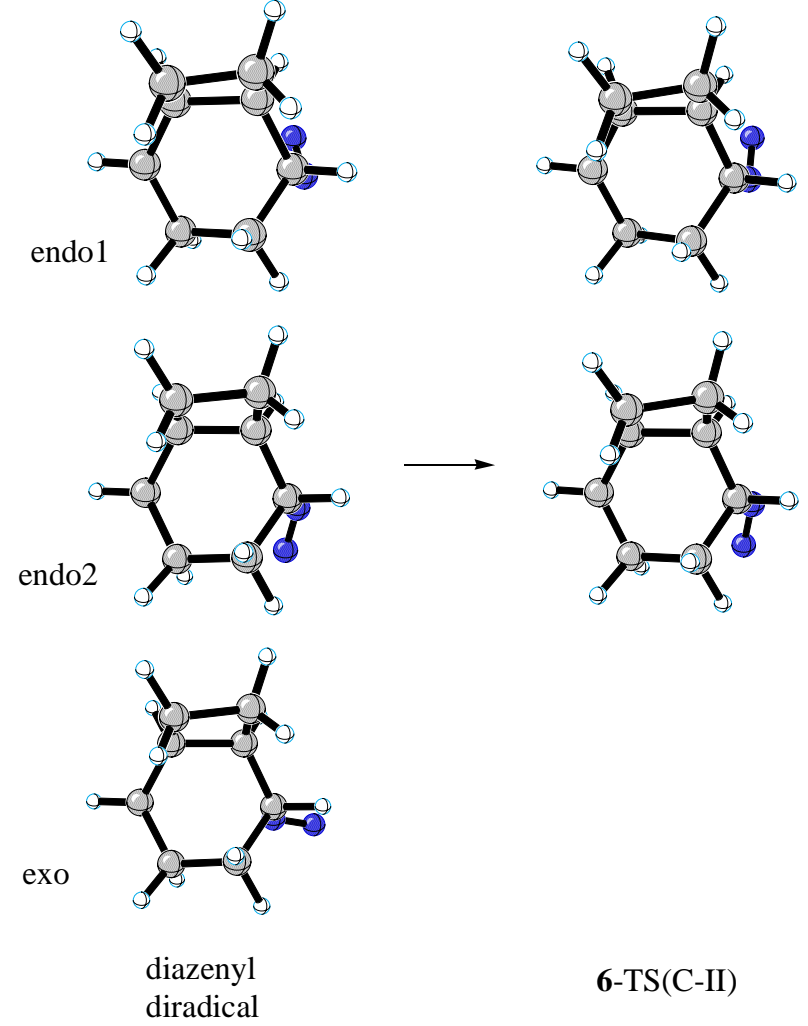

Intermediate from 6: exo but diazenyl diradical, endo1 $\mathrm{N}=\mathrm{N}$

UB3LYP/6-31G(d)

$E(R B+H F-L Y P)=-421.438610093$

Sum of electronic and zero-point Energies= $\quad-421.251197$

Sum of electronic and thermal Energies= $\quad-421.241575$

Sum of electronic and thermal Enthalpies= $\quad-421.240631$

Sum of electronic and thermal Free Energies $\quad-421.286350$

KCAL/MOL

E (Thermal)
KCAL/MOL

123.129
CV CAL/MOL-KELVIN CAL/MOL-KELVIN 36.657
96.172

$\begin{array}{rl}1 & 6 \\ 2 & 6 \\ 3 & 6 \\ 4 & 1 \\ 5 & 1 \\ 6 & 6 \\ 7 & 1 \\ 8 & 6 \\ 9 & 1 \\ 10 & 1 \\ 11 & 6 \\ 12 & 1 \\ 13 & 7 \\ 14 & 7 \\ 15 & 6\end{array}$

$$
\begin{array}{r}
-.776264 \\
-.647705 \\
-.556611 \\
-1.501479 \\
.251342 \\
.412287 \\
-1.563552 \\
.379360 \\
.645994 \\
-.900493 \\
1.074824 \\
-.301724 \\
-2.162818 \\
-2.323533 \\
2.364246
\end{array}
$$
$-.583814$
.831750
1.886346
1.043590
.865040
1.513697
2.025036
- . 995130
2. 252034
$-1.307379$
.186661
2. 864429
$-.647672$
$-1.044511$
$-.038941$

.730174

1. 297972

.181329

1.950781

1. 927209

- . 898674

$-.250475$

- . 177616

$-1.662275$

1.544999

$-.948579$

.619030

.019037

$-1.074863$

$-.056098$ 


$\begin{array}{rrrrr}16 & 6 & 1.714982 & -1.303187 & .562091 \\ 17 & 1 & 3.291414 & -.197312 & -.615454 \\ 18 & 1 & 2.530803 & .769343 & .662268 \\ 19 & 1 & 2.174080 & -2.241574 & .236197 \\ 20 & 1 & 1.659004 & -1.321033 & 1.656625 \\ 21 & 1 & .039336 & -1.800958 & -.834656 \\ 22 & 1 & 1.289032 & -.090233 & -1.987061\end{array}$

CASPT2 $(12,12) / 6-31 G * / / U B 3 L Y P / 6-31 G$ *

Reference energy: $\quad-418.8207664804$

Total energy: $\quad-420.0516587746$

Intermediate from 6: exo but diazenyl dirad, endo2 $\mathrm{N}=\mathrm{N}$

\begin{tabular}{|c|c|c|c|c|c|c|}
\hline \multicolumn{7}{|c|}{ UB3LYP/6-31G(d) } \\
\hline $\mathrm{E}(\mathrm{UB}+\mathrm{H}$ & $F-L Y P)=-L$ & 421.438522356 & & & & \\
\hline Sum of & electronic & and zero-point & nergies= & & -421 & 251280 \\
\hline Sum of & electronic & and thermal Ene & gies= & & -421 & 241682 \\
\hline Sum of & electronic & and thermal Ent & alpies= & & -421 & 240737 \\
\hline Sum of & electronic & and thermal Fre & Energies= & & -421 & 286418 \\
\hline & & E (Thermal) & & & & $\mathrm{S}$ \\
\hline & & $\mathrm{KCAL} / \mathrm{MOL}$ & $\mathrm{CAL} / \mathrm{MOL}$ & ELVIN & & /MOL-KELVIN \\
\hline TOTAL & & 123.519 & & 626 & & 96.142 \\
\hline 1 & 6 & & -0.683202 & -0.85 & 5490 & $\odot .427631$ \\
\hline 2 & 6 & & -0.799316 & 0.42 & 8596 & 1.248753 \\
\hline 3 & 6 & & -0.721234 & 1.69 & 2158 & 0.374078 \\
\hline 4 & 1 & & -1.736818 & 0.42 & 2249 & 1.815179 \\
\hline 5 & 1 & & 0.016541 & 0.42 & 5819 & 1.983933 \\
\hline 6 & 6 & & 0.364557 & 1.63 & 4480 & -0.653158 \\
\hline 7 & 1 & & -1.698447 & 1.83 & 2475 & -0.119644 \\
\hline 8 & 6 & & 0.594027 & -0.95 & 2083 & $-\odot .404959$ \\
\hline 9 & 1 & & 0.579328 & 2.53 & 1848 & -1.229230 \\
\hline 10 & 1 & & -0.762531 & -1.74 & 2399 & 1.068409 \\
\hline 11 & 6 & & 1.196310 & 0.42 & 4996 & -0.866943 \\
\hline 12 & 1 & & -0.604475 & 2.57 & 6312 & 1.021182 \\
\hline 13 & 7 & & -1.899832 & -1.05 & 3159 & -0.534747 \\
\hline 14 & 7 & & -2.869390 & -0.39 & 0510 & -0.503705 \\
\hline 15 & 6 & & 2.420016 & 0.17 & 6472 & 0.108893 \\
\hline 16 & 6 & & 1.892163 & -1.25 & 0250 & $\odot .4 \odot 3497$ \\
\hline 17 & 1 & & 3.406974 & 0.24 & 0104 & -0.359467 \\
\hline 18 & 1 & & 2.409205 & 0.83 & 9788 & 0.979014 \\
\hline 19 & 1 & & 2.496362 & -2.04 & 5448 & -0.043589 \\
\hline 20 & 1 & & 1.745589 & -1.49 & 5305 & 1.461597 \\
\hline 21 & 1 & & 0.414761 & -1.65 & 1282 & -1.227598 \\
\hline 22 & 1 & & 1.538131 & 0.37 & 8254 & -1.907373 \\
\hline
\end{tabular}

Intermediate from 6: exo but diazenyl dirad, exo $\mathrm{N}=\mathrm{N}$

UB3LYP/6-31G(d)

$\mathrm{E}(\mathrm{UB}+\mathrm{HF}-\mathrm{LYP})=-421.438449527$

Sum of electronic and zero-point Energies= $\quad-421.251119$

Sum of electronic and thermal Energies= -421.241454

Sum of electronic and thermal Enthalpies= $\quad-421.240510$

Sum of electronic and thermal Free Energies= $\quad-421.286520$ 
TOTAL

$\begin{array}{rr}1 & 6 \\ 2 & 6 \\ 3 & 6 \\ 4 & 1 \\ 5 & 1 \\ 6 & 6 \\ 7 & 1 \\ 8 & 6 \\ 9 & 1 \\ 10 & 1 \\ 11 & 6 \\ 12 & 1 \\ 13 & 7 \\ 14 & 7 \\ 15 & 6 \\ 16 & 6 \\ 17 & 1 \\ 18 & 1 \\ 19 & 1 \\ 20 & 1 \\ 21 & 1 \\ 22 & 1\end{array}$

E (Thermal) $\mathrm{KCAL} / \mathrm{MOL}$ 123.617
CV CAL/MOL-KELVIN CAL/MOL-KELVIN 36.609
$S$ 96.836

$$
\begin{array}{r}
-0.516308 \\
0.802429 \\
1.948353 \\
1.065085 \\
0.638074 \\
1.561429 \\
2.272005 \\
-0.979668 \\
2.334871 \\
-1.308135 \\
0.167550 \\
2.826092 \\
-0.285726 \\
-0.898052 \\
-0.333363 \\
-1.559912 \\
-0.549746 \\
0.356334 \\
-2.505658 \\
-1.704702 \\
-1.654204 \\
-0.026634
\end{array}
$$

0.463681

1.205035

$\odot .246719$

1.785712

1.930596

$-0.748794$

$-0.282557$

$-0.355269$

$-1.387194$

1.152853

$-0.878832$

0.832069

$-0.514961$

$-0.429097$

0.133292

0.489194

$-0.316854$

0.968554

0.091169

1.555137

$-1.143550$

$-1.907690$

Transition structure 6-TS(C-II): cleave 2nd $\mathrm{CN}$, endo1 $\mathrm{N}=\mathrm{N}$

UB3LYP/6-31G(d)

$\mathrm{E}(\mathrm{UB}+\mathrm{HF}-\mathrm{LYP})=-421.438246142$

Sum of electronic and zero-point Energies=

Sum of electronic and thermal Energies=

Sum of electronic and thermal Enthalpies=

Sum of electronic and thermal Free Energies=

$-421.251723$

$-421.242108$

$-421.241164$

$-421.287170$

E (Thermal) $\mathrm{KCAL} / \mathrm{MOL}$

TOTAL 122.355
CV CAL/MOL-KELVIN CAL/MOL-KELVIN 35.889
$\mathrm{S}$ 96.829

$\begin{array}{rl}1 & 6 \\ 2 & 6 \\ 3 & 6 \\ 4 & 1 \\ 5 & 1 \\ 6 & 6 \\ 7 & 6 \\ 8 & 1 \\ 9 & 6 \\ 10 & 1 \\ 11 & 1 \\ 12 & 6 \\ 13 & 1 \\ 14 & 6\end{array}$

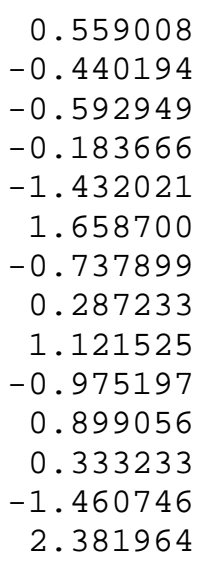

1.495754

1. 946997

0.959965

2. 945641

2. 070266

$-1.419767$

$-0.467648$

1. 017592

0.122862

$-1.171061$

2. 209697

$-0.985614$

1. 230383

$-0.180191$
$-0.929569$

0.089591

1. 261091

0.474716

$-0.381924$

$\odot .555533$

$\odot .777875$

1. 918549

$-0.936015$

1.581004

$-1.676480$

$-0.151454$

1.873184

$-0.025816$ 


$\begin{array}{rrrrr}15 & 1 & 3.315867 & -0.367333 & -0.564677 \\ 16 & 1 & 2.562743 & 0.605870 & 0.713695 \\ 17 & 1 & 2.042720 & -2.369521 & 0.170057 \\ 18 & 1 & 1.621055 & -1.489425 & 1.648250 \\ 19 & 1 & 1.330323 & -0.200027 & -1.962997 \\ 20 & 1 & -0.098874 & -1.761163 & -0.793029 \\ 21 & 7 & -2.302621 & -0.480030 & -0.037842 \\ 22 & 7 & -2.498638 & -1.170694 & -0.940410\end{array}$

CASPT2 $(12,12) / 6-31 G * / / U B 3 L Y P / 6-31 G$ *

Reference energy: $\quad-418.8253200258$

Total energy: $\quad-420.0486381507$

Transition structure 6-TS(C-II): cleave 2nd CN, endo2 N=N

UB3LYP/6-31G(d)

$E(U B+H F-L Y P)=-421.436887044$

Sum of electronic and zero-point Energies= $\quad-421.251456$

Sum of electronic and thermal Energies= $\quad-421.241933$

Sum of electronic and thermal Enthalpies= $\quad-421.240989$

Sum of electronic and thermal Free Energies $=\quad-421.286584$

$\begin{array}{rr}\text { KCAL/MOL } \\ \text { TOTAL } & 122.335\end{array}$

E (Thermal)
KCAL/MOL
CV
CAL/MOL-KELVIN CAL/MOL-KELVIN 35.854
95.962

$\begin{array}{rr}1 & 6 \\ 2 & 6 \\ 3 & 6 \\ 4 & 1 \\ 5 & 1 \\ 6 & 6 \\ 7 & 6 \\ 8 & 1 \\ 9 & 6 \\ 10 & 1 \\ 11 & 1 \\ 12 & 6 \\ 13 & 1 \\ 14 & 6 \\ 15 & 1 \\ 16 & 1 \\ 17 & 1 \\ 18 & 1 \\ 19 & 1 \\ 20 & 1 \\ 21 & 7 \\ 22 & 7\end{array}$

0.423260
-0.705681
-0.796826
-0.635077
-1.666217
1.923435
-0.621410
-0.015571
1.228877
-0.768926
0.689690
0.597448
-1.757820
2.442200
3.438155
2.399177
2.507550
1.817084
1.581677
0.362477
-2.017775
-2.967941

1.640421

1. 716024

๑. 487637

2.629480

1. 810661

$-1.298258$

$-0.817895$

0.542035

0.411145

$-1.700143$

2.538023

$-0.948752$

○. 486585

0.141310

๑. 239197

0.768616

$-2.060209$

$-1.613341$

๑. 342903

$-1.634740$

$-1.059595$

$-0.417385$
$-0.659981$

๑. 317020

1. 241303

0.927845

$-0.219648$

๑. 363087

๑. 494708

2. 014368

$-0.853430$

1. 124001

$-1.213918$

$-0.388113$

1. 768303

○. 129483

- 0.313195

1.025013

$-0.162375$

1.407015

$-1.889534$

$-1.208952$

$-0.569612$

$-0.448013$ 

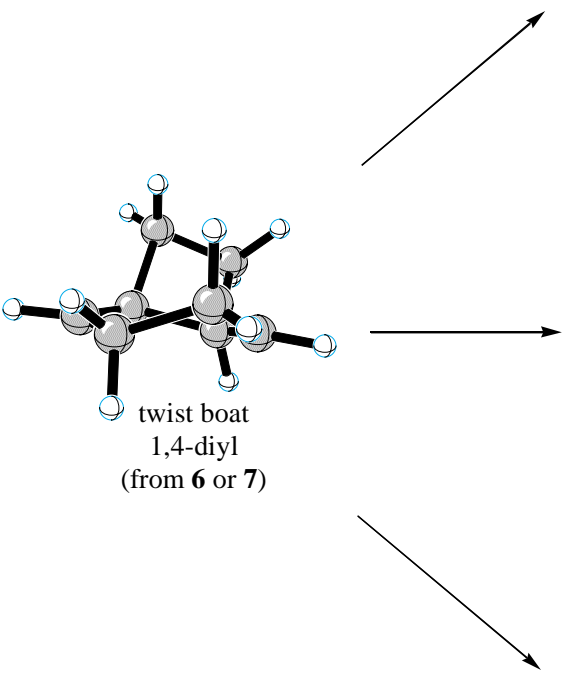

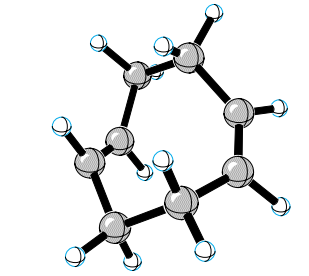

1,5-cis,trans-cyclooctadiene

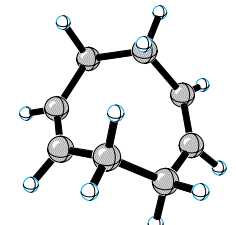

1,5-cis,cis-cyclooctadiene

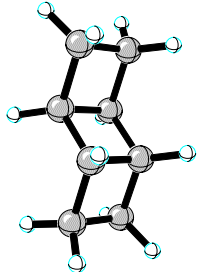

tricyclooctane

Intermediate from 6 or 7: twist boat 1,4-diyl

UB3LYP/6-31G(d)

$E(U B+H F-L Y P)=-311.937049033$

Sum of electronic and zero-point Energies= $\quad-311.760932$

Sum of electronic and thermal Energies=

$-311.753091$

Sum of electronic and thermal Enthalpies=

$-311.752147$

Sum of electronic and thermal Free Energies=

$-311.793162$

TOTAL

$$
\text { E (Thermal) }
$$
$\mathrm{KCAL} / \mathrm{MOL}$

115.435
CV

CAL/MOL-KELVIN

30.503
$\mathrm{S}$

CAL/MOL-KELVIN

86.323

$\begin{array}{rl}1 & 6 \\ 2 & 6 \\ 3 & 6 \\ 4 & 1 \\ 5 & 1 \\ 6 & 6 \\ 7 & 6 \\ 8 & 1 \\ 9 & 6 \\ 10 & 1 \\ 11 & 1 \\ 12 & 6 \\ 13 & 1\end{array}$
0.852467
1.998467
1.539508
2.725409
2.556502
$-2.001044$
0.397288
1.259816
$-0.525868$
๑. 291601
1.031470
$-0.722996$
2. 382859

$-0.232764$

0.002770

$\odot .537499$

0.694900

$-0.936275$

0.187568

$-0.249475$

1.601124

$-0.473788$

$-0.331833$

$-0.207760$

$-0.736733$

0.536384 


$\begin{array}{rrrrr}14 & 6 & -1.530449 & -0.793544 & 0.741295 \\ 15 & 1 & -2.278190 & -1.587385 & 0.837078 \\ 16 & 1 & -0.997709 & -0.698058 & 1.692545 \\ 17 & 1 & -2.919303 & 0.486838 & -0.403893 \\ 18 & 1 & -2.117121 & 1.390142 & 0.902600 \\ 19 & 1 & -1.016271 & -1.500351 & -1.263878 \\ 20 & 1 & -0.963300 & 0.869168 & -1.779227\end{array}$

Product from 6 or 7 : cis, trans-1,5-cyclooctadiene

RB3LYP/6-31G(d)

$\mathrm{E}(\mathrm{UB}+\mathrm{HF}-\mathrm{LYP})=-312.007478231$

Sum of electronic and zero-point Energies= $\quad-311.826767$

Sum of electronic and thermal Energies= $\quad-311.819536$

Sum of electronic and thermal Enthalpies $=\quad-311.818592$

Sum of electronic and thermal Free Energies= $\quad-311.857631$

$\begin{array}{lr} & \mathrm{KCAL} / \mathrm{MOL} \\ \text { TOTAL } & 117.936\end{array}$

E (Thermal)

$\begin{array}{rl}1 & 6 \\ 2 & 6 \\ 3 & 1 \\ 4 & 6 \\ 5 & 1 \\ 6 & 1 \\ 7 & 6 \\ 8 & 6 \\ 9 & 1 \\ 10 & 6 \\ 11 & 1 \\ 12 & 1 \\ 13 & 6 \\ 14 & 1 \\ 15 & 1 \\ 16 & 6 \\ 17 & 1 \\ 18 & 1 \\ 19 & 1 \\ 20 & 1\end{array}$

CV

CAL/MOL-KELVIN 29.433
$S$

CAL/MOL-KELVIN

82.166

$\begin{array}{rrr}0.461113 & -1.449891 & 0.211541 \\ 1.810275 & -0.874124 & -0.096662 \\ -1.153360 & 2.189852 & -0.924874 \\ 1.713972 & 0.614650 & 0.404502 \\ 2.639912 & -1.372865 & 0.419705 \\ 2.020792 & -0.887692 & -1.172844 \\ -1.578750 & 0.633562 & 0.589129 \\ 0.697411 & 1.472173 & -0.335381 \\ 1.476663 & 0.584767 & 1.476821 \\ -0.577343 & -1.238431 & -0.603918 \\ 1.138694 & 2.163850 & -1.054259 \\ 0.264090 & -1.624918 & 1.272900 \\ -0.646550 & 1.488764 & -0.259695 \\ -0.376328 & -1.004853 & -1.648914 \\ 2.702282 & 1.084642 & 0.321262 \\ -1.901534 & -0.758837 & -0.059338 \\ -2.505757 & 1.186304 & 0.792416 \\ -1.107009 & 0.433884 & 1.558617 \\ -2.653099 & -0.657576 & -0.850336 \\ -2.318446 & -1.422591 & 0.708440\end{array}$

Product from 6 or 7: 1,4-cis,cis-cyclooctadiene

RB3LYP/6-31G(d)

$E(R B+H F-L Y P)=-312.030426419$

Sum of electronic and zero-point Energies= $\quad-311.849219$

Sum of electronic and thermal Energies= $\quad-311.841807$

Sum of electronic and thermal Enthalpies= -311.840863

Sum of electronic and thermal Free Energies= $\quad-311.880059$

$\mathrm{KCAL} / \mathrm{MOL}$

$$
\begin{gathered}
\mathrm{E}(\text { Thermal }) \\
\text { KCAL/MOL } \\
118.361
\end{gathered}
$$

CV

CAL/MOL-KELVIN CAL/MOL-KELVIN $29.311 \quad 82.495$ 


$\begin{array}{rrrrr}1 & 1 & -2.641694 & -.150419 & -.722929 \\ 2 & 6 & -1.700858 & .083624 & -.223555 \\ 3 & 6 & .113739 & 1.920188 & -.017658 \\ 4 & 6 & -.113739 & -1.920188 & -.017658 \\ 5 & 6 & 1.166757 & 1.019840 & .664553 \\ 6 & 6 & -1.166757 & -1.019840 & .664553 \\ 7 & 6 & -1.166757 & 1.280286 & -.496724 \\ 8 & 1 & .584260 & 2.406578 & -.885133 \\ 9 & 1 & -.584260 & -2.406578 & -.885133 \\ 10 & 1 & 2.006066 & 1.663468 & .956682 \\ 11 & 1 & -.766782 & -.620821 & 1.599981 \\ 12 & 1 & 1.734958 & -1.918867 & -1.175391 \\ 13 & 6 & 1.166757 & -1.280286 & -.496724 \\ 14 & 1 & -.138902 & 2.746061 & .667098 \\ 15 & 1 & 2.641694 & .150419 & -.722929 \\ 16 & 6 & 1.700858 & -.083624 & -.223555 \\ 17 & 1 & -2.006066 & -1.663468 & .956682 \\ 18 & 1 & -1.734958 & 1.918867 & -1.175391 \\ 19 & 1 & .138902 & -2.746061 & .667098 \\ 20 & 1 & .766782 & .620821 & 1.599981\end{array}$

\section{Product from 6 or 7: tricyclooctane}

RB3LYP/6-31G(d)

$E(R B+H F-L Y P)=-311.987425566$

Sum of electronic and zero-point Energies= $\quad-311.806461$

Sum of electronic and thermal Energies=

Sum of electronic and thermal Enthalpies=

Sum of electronic and thermal Free Energies=

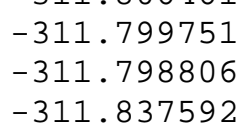

CV

CAL/MOL-KELVIN CAL/MOL-KELVIN 26.883
81.630
$-1.261784$
$-.778774$
$-.791066$
.778594
.791234
.790956
1.405975
$-1.212026$
1. 210783
1. 262470
$-1.405285$
$-.791480$
$-.778461$
1. 404480
.778888
$-1.405348$
$-1.261847$
1. 211840
1.262302
$-1.210896$ 


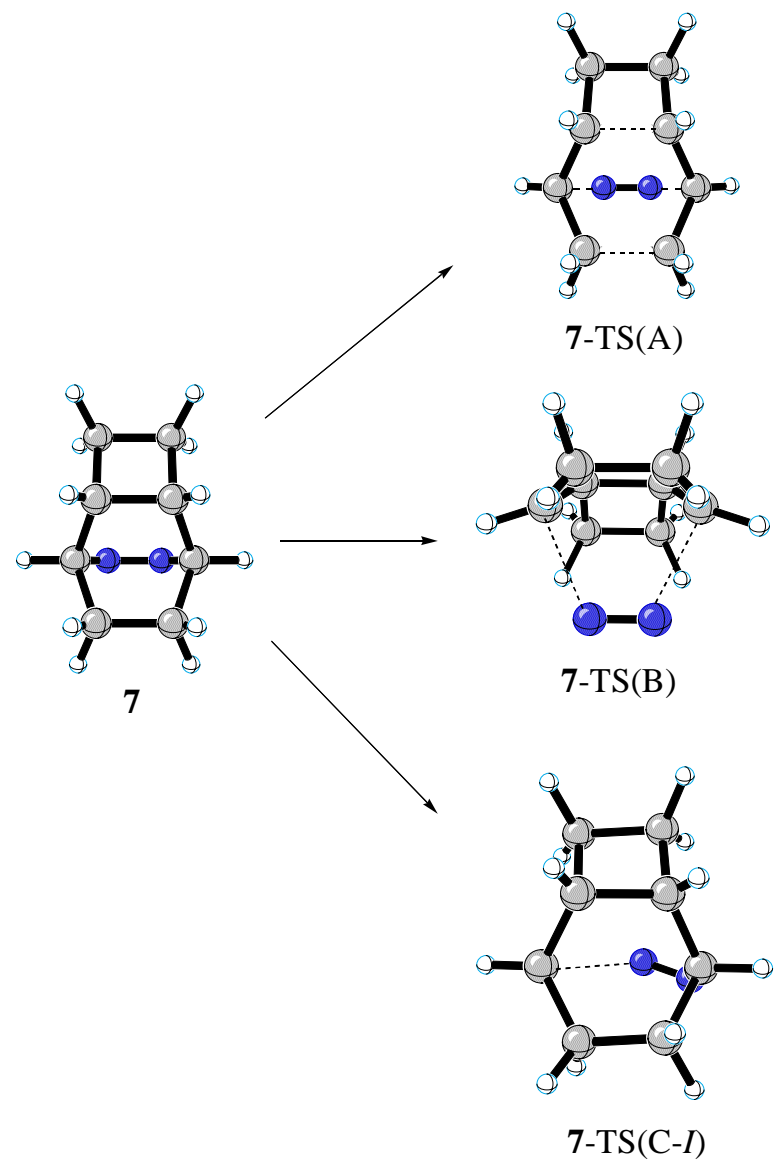

Reactant 7: endo_cyclobutane DBO

RB3LYP/6-31G(d)

$E(R B+H F-L Y P)=-421.503211921$

Sum of electronic and zero-point Energies= $\quad-421.309007$

Sum of electronic and thermal Energies= $\quad-421.301536$

Sum of electronic and thermal Enthalpies= -421.300591

Sum of electronic and thermal Free Energies= $\quad-421.340962$

E (Thermal)
KCAL/MOL

CV $S$

TOTAL

126.554
CAL/MOL-KELVIN CAL/MOL-KELVIN
31.287
84.967

$\begin{array}{rl}1 & 6 \\ 2 & 1 \\ 3 & 1 \\ 4 & 6 \\ 5 & 1 \\ 6 & 6 \\ 7 & 7 \\ 8 & 6 \\ 9 & 1 \\ 10 & 1 \\ 11 & 1 \\ 12 & 6\end{array}$

0.515972
-0.671302
2.680201
-2.090979
2.680199
-0.694570
0.483762
-0.694571
-2.108710
1.840968
-2.882889
1.810050

$-1.258430$

0.104092

$1.252339-1.703137$

$-1.170258-0.061640$

$-0.779142-0.025606$

$1.170262-0.061640$

$-0.778601-0.715197$

$-0.625810 \quad 1.453622$

$0.778599 \quad-0.715197$

$1.237488 \quad 0.967164$

$1.181252-1.612579$

$-1.231863-0.629715$

$-0.775518-0.595069$ 


$\begin{array}{rrrrr}13 & 6 & 1.810049 & 0.775522 & -0.595069 \\ 14 & 1 & 1.840970 & -1.181248 & -1.612579 \\ 15 & 6 & 0.515969 & 1.258430 & 0.104092 \\ 16 & 7 & 0.483761 & 0.625810 & 1.453622 \\ 17 & 1 & 0.512578 & 2.341230 & 0.255232 \\ 18 & 1 & 0.512583 & -2.341230 & 0.255232 \\ 19 & 1 & -0.671300 & -1.252341 & -1.703137 \\ 20 & 6 & -2.090980 & 0.779138 & -0.025606 \\ 21 & 1 & -2.882891 & 1.231857 & -0.629715 \\ 22 & 1 & -2.108708 & -1.237492 & 0.967164\end{array}$

CASPT2 $(12,12) / 6-31 G * / / B 3 L Y P / 6-31 G *$

Reference energy: $\quad-418.8838318154$

Total energy: $\quad-420.1220796821$

CASPT2 $(6,6) / 6-31 G * / / B 3 L Y P / 6-31 G *$

Reference energy: $\quad-418.8332858370$

Total energy: $\quad-420.1207013919$

\section{Transition structure 7-TS $(A)$ : pericyclic loss of nitrogen}

RB3LYP/6-31G(d)

$E(R B+H F-L Y P)=-421.416432065$

Sum of electronic and zero-point Energies= -421.231101

Sum of electronic and thermal Energies= $\quad-421.221677$

Sum of electronic and thermal Enthalpies= $\quad-421.220733$

Sum of electronic and thermal Free Energies= $\quad-421.265396$

KCAL/MOL
TOTAL
122.211

E (Thermal) CV S

CAL/MOL-KELVIN CAL/MOL-KELVIN 36.741
94.001

$\begin{array}{rrrrr}1 & 6 & 0.720398 & -0.776092 & -0.843111 \\ 2 & 1 & -2.623170 & -0.205562 & -1.226261 \\ 3 & 6 & 0.720348 & -0.774672 & 0.844349 \\ 4 & 7 & -0.574764 & 1.897722 & -0.568301 \\ 5 & 1 & -0.477688 & 0.461702 & -2.264751 \\ 6 & 6 & -1.741582 & -0.734004 & 0.853039 \\ 7 & 1 & -1.871735 & -1.800364 & 1.069909 \\ 8 & 6 & 2.057951 & 0.002025 & 0.782989 \\ 9 & 1 & 2.907289 & -0.520258 & 1.234649 \\ 10 & 1 & 2.907559 & -0.523448 & -1.232981 \\ 11 & 1 & 0.834585 & -1.834242 & 1.102279 \\ 12 & 1 & -2.623600 & -0.205712 & 1.226549 \\ 13 & 6 & -0.493600 & -0.223868 & -1.433051 \\ 14 & 6 & -0.493950 & -0.222068 & 1.433369 \\ 15 & 6 & -1.741652 & -0.734464 & -0.852411 \\ 16 & 1 & -0.478498 & 0.464502 & 2.264239 \\ 17 & 7 & -0.575184 & 1.898432 & 0.565889 \\ 18 & 1 & -1.872855 & -1.800824 & -1.068651 \\ 19 & 6 & 2.058251 & 0.000275 & -0.782911 \\ 20 & 1 & 1.997773 & 1.003595 & 1.218439 \\ 21 & 1 & 1.998773 & 1.000855 & -1.220731 \\ 22 & 1 & 0.834215 & -1.836112 & -1.099391\end{array}$

CASPT2 $(12,12) / 6-31 G * / / B 3 L Y P / 6-31 G *$ 
$\begin{array}{ll}\text { Reference energy: } & -418.8292991865 \\ \text { Total energy: } & -420.0385255480\end{array}$

Transition structure 7-TS(B): 2-bond CN cleavage

UB3LYP/6-31G(d)

$E(U B+H F-L Y P)=-421.430496038$

Sum of electronic and zero-point Energies $=\quad-421.245223$

Sum of electronic and thermal Energies= $\quad-421.235926$

Sum of electronic and thermal Enthalpies $=\quad-421.234982$

Sum of electronic and thermal Free Energies $\quad-421.280123$

\begin{tabular}{|c|c|c|c|c|c|}
\hline \multirow{2}{*}{$\begin{array}{c}\text { TOTAL } \\
1\end{array}$} & \multicolumn{2}{|r|}{$\begin{array}{c}\text { E (Thermal) } \\
\text { KCAL/MOL } \\
122.094\end{array}$} & $\begin{array}{r}\mathrm{CAL} / \mathrm{MOL} \\
3\end{array}$ & $\begin{array}{l}\text { LVIN } \\
42\end{array}$ & $\begin{array}{c}\mathrm{S} \\
\text { MOL-KELVIN } \\
95.008\end{array}$ \\
\hline & 6 & & ๑.505912 & -1.371058 & -0.215446 \\
\hline 2 & 6 & & 1.807641 & $-\odot .772691$ & -0.667373 \\
\hline 3 & 1 & & -0.841075 & 1.233239 & -1.794942 \\
\hline 4 & 6 & & 1.811254 & $\odot .809939$ & -0.613134 \\
\hline 5 & 1 & & 2.633549 & -1.176119 & $-\odot .071047$ \\
\hline 6 & 1 & & 2.006687 & -1.080432 & -1.706860 \\
\hline 7 & 6 & & -2.060116 & -0.778925 & -0.024111 \\
\hline 8 & 6 & & 0.502455 & 1.382330 & $-\odot .148504$ \\
\hline 9 & 1 & & 2.623086 & 1.167337 & 0.030350 \\
\hline 10 & 7 & & 0.483734 & $\odot .522288$ & 1.828804 \\
\hline 11 & 6 & & -0.720713 & -0.771627 & $-\odot .819758$ \\
\hline 12 & 1 & & 0.481132 & 2.406074 & 0.213483 \\
\hline 13 & 7 & & $\odot .490096$ & -0.621448 & 1.796863 \\
\hline 14 & 1 & & $\odot .489368$ & -2.413592 & $\odot .088573$ \\
\hline 15 & 6 & & -0.721175 & 0.810602 & -0.783883 \\
\hline 16 & 1 & & -0.840223 & -1.147569 & -1.849077 \\
\hline 17 & 1 & & -1.994337 & 1.211286 & 1.014336 \\
\hline 18 & 6 & & -2.060691 & $\odot .780757$ & 0.011393 \\
\hline 19 & 1 & & 2.038910 & 1.187257 & -1.623265 \\
\hline 20 & 1 & & -2.903828 & 1.245567 & -0.508786 \\
\hline 21 & 1 & & $-2.9 \odot 2688$ & -1.219823 & -0.565618 \\
\hline 22 & 1 & & -1.994790 & -1.255069 & ๑. 958078 \\
\hline
\end{tabular}

CASPT2 $(12,12) / 6-31 G * / / U B 3 L Y P / 6-31 G *$

Reference energy: $\quad-418.8303267679$

Total energy: $\quad-420.0472062508$

CASPT2 $(6,6) / 6-31 G * / / U B 3 L Y P / 6-31 G$ *

Reference energy: $\quad-418.7784320441$

Total energy: $\quad-420.0454758289$

Transition structure 7-TS(C-I): 2-bond CN cleavage

UB3LYP/6-31G*

$E(U B+H F-L Y P)=-421.433960056$

Sum of electronic and zero-point Energies $=\quad-421.246363$

Sum of electronic and thermal Energies= $\quad-421.237735$

Sum of electronic and thermal Enthalpies= $\quad-421.236791$ 
E (Thermal) $\mathrm{KCAL} / \mathrm{MOL}$

TOTAL

$\begin{array}{rl}1 & 6 \\ 2 & 6 \\ 3 & 6 \\ 4 & 1 \\ 5 & 1 \\ 6 & 6 \\ 7 & 6 \\ 8 & 1 \\ 9 & 6 \\ 10 & 1 \\ 11 & 1 \\ 12 & 6 \\ 13 & 1 \\ 14 & 6 \\ 15 & 1 \\ 16 & 1 \\ 17 & 1 \\ 18 & 1 \\ 19 & 1 \\ 20 & 1 \\ 21 & 7 \\ 22 & 7\end{array}$

CV CAL/MOL-KELVIN 34.325

0.651081

1.827366

1.748467

2.781194

1.787251

$-2.083776$

$\odot .349059$

2. 202153

$-0.664631$

0.190278

0.786310

$-0.818607$

2. 391755

$-1.998108$

$-2.801622$

$-1.942655$

$-2.997794$

$-1.911737$

$-0.725100$

$-1.114020$

0.757528

$\odot .283740$
$S$ CAL/MOL-KELVIN 90.053
$-0.792283$
$-0.825424$
0.313697
$-0.784062$
$-1.800833$
0.494379
0.617993
1.223550
$-1.021761$
1.469536
$-1.552009$
$-0.219922$
0.052196
$-0.407590$
$-1.147298$
0.125024
0.391675
1.552144
$-2.106819$
$-0.891913$
0.527934
1.545534

CASPT2 $(12,12) / 6-31 G * / / U B 3 L Y P / 6-31 G^{*}$

Reference energy:

Total energy:
$-418.8161150667$

$-420.0490724274$ 


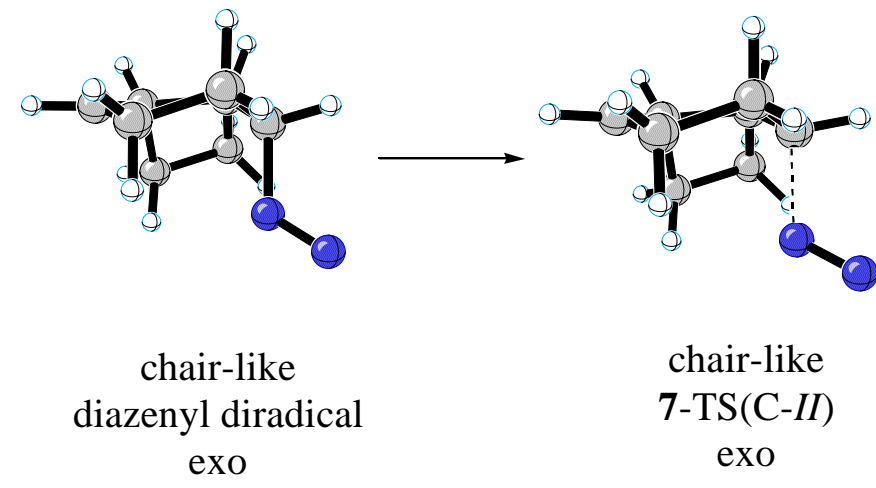

Intermediate from 7: endo but diazenyl diradical, chair-like, exo $\mathrm{N}=\mathrm{N}$

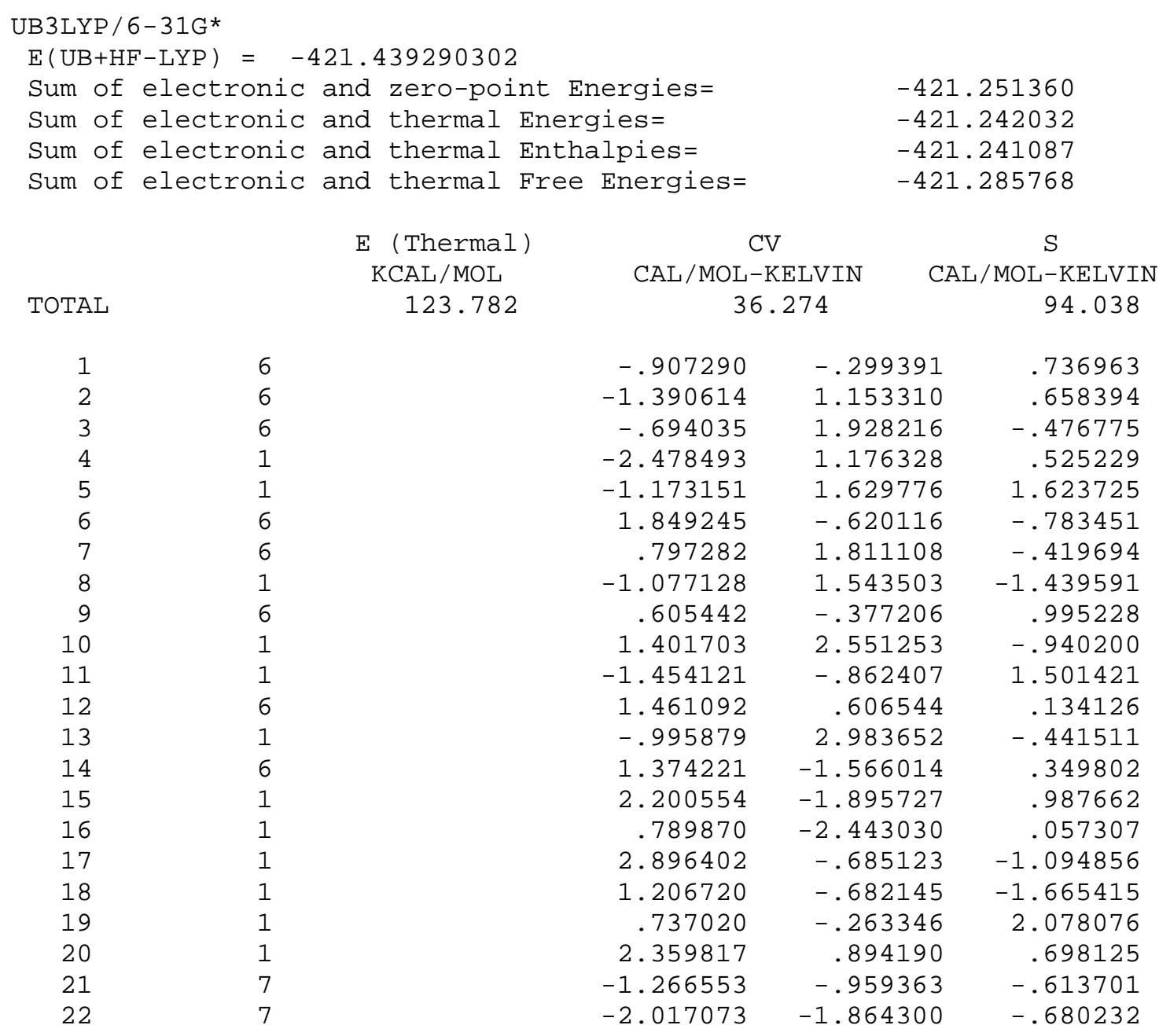

CASPT2 $(12,12) / 6-31 G * / / U B 3 L Y P / 6-31 G$ *

Reference energy: $\quad-418.8221157562$

Total energy: $\quad-420.0547172872$ 
Intermediate from 7: endo but diazenyl diradical, chair-like, endo $\mathrm{N}=\mathrm{N}$

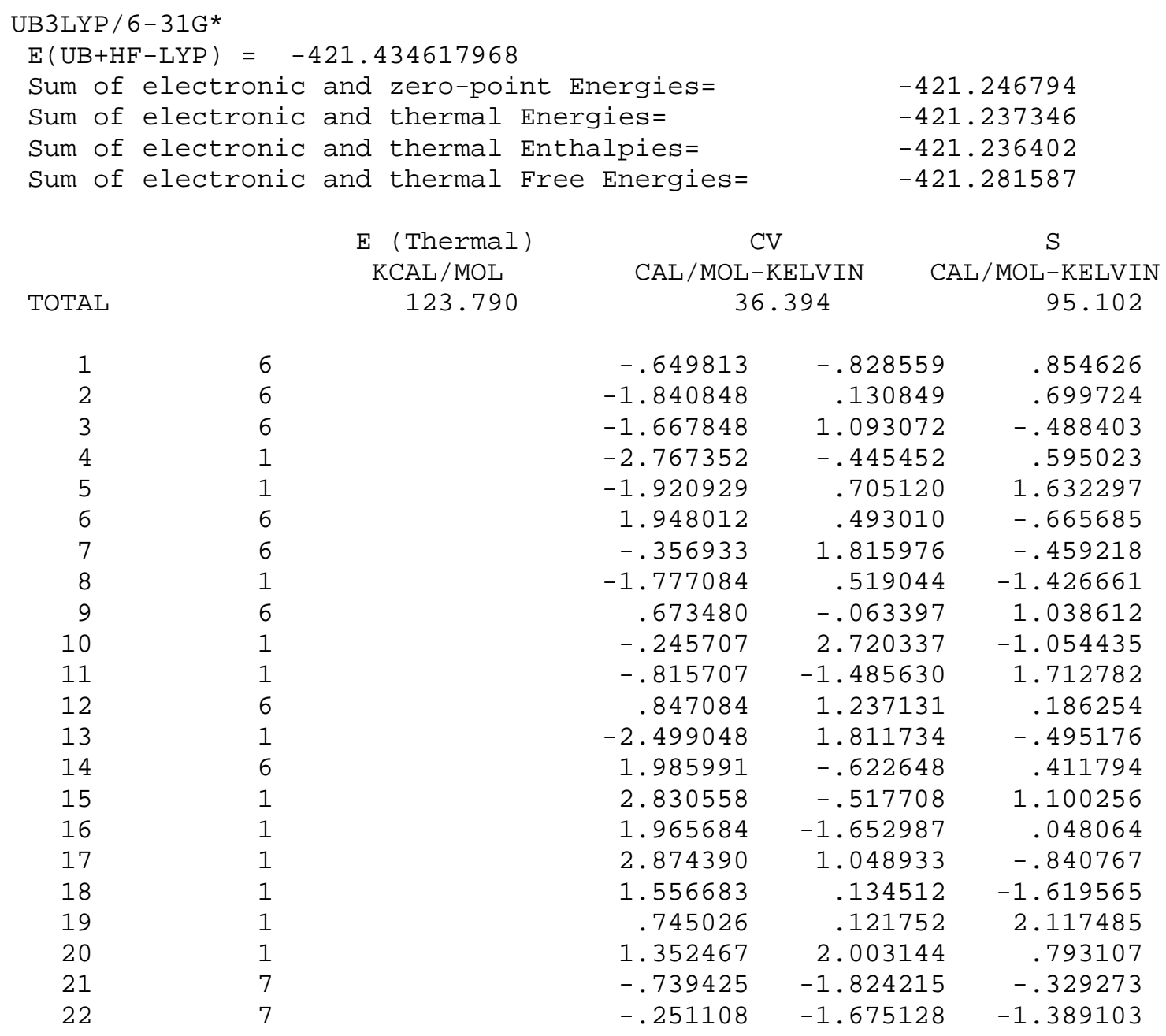

Transition structure 7-TS(C-II): cleave 2nd CN bond, chair-like, exo N=N

UB3LYP/6-31G*

$\mathrm{E}(\mathrm{UB}+\mathrm{HF}-\mathrm{LYP})=-421.436293753$

Sum of electronic and zero-point Energies= $\quad-421.250723$

Sum of electronic and thermal Energies= -421.241280

Sum of electronic and thermal Enthalpies= $\quad-421.240336$

Sum of electronic and thermal Free Energies= $\quad-421.286008$

$\begin{array}{cccc} & \text { KCAL/MOL } & \text { CAL/MOL }- \text { KELVIN } & \text { CAL/MOL-KELVIN } \\ \text { TOTAL } & 122.373 & 35.784 & 96.124\end{array}$

$\begin{array}{ccc}\text { E (Thermal) } & \text { CV } & \mathrm{S} \\ \text { KCAL/MOL } & \text { CAL/MOL-KELVIN } & \text { CAL/MOL-KELVIN } \\ 122.373 & 35.784 & 96.124\end{array}$

$\begin{array}{rrrrr}1 & 6 & -.842625 & -.139022 & .864457 \\ 2 & 6 & -1.190121 & 1.326467 & .697947 \\ 3 & 6 & -.442725 & 1.959921 & -.495015 \\ 4 & 1 & -2.273568 & 1.445778 & .579253 \\ 5 & 1 & -.911954 & 1.854235 & 1.623015 \\ 6 & 6 & 1.728109 & -.868478 & -.826311 \\ 7 & 6 & 1.026650 & 1.682811 & -.461360\end{array}$




$\begin{array}{rrrrr}8 & 1 & -.891867 & 1.563024 & -1.424067 \\ 9 & 6 & .635353 & -.432449 & 1.015084 \\ 10 & 1 & 1.699952 & 2.354492 & -.989925 \\ 11 & 1 & -1.465404 & -.669076 & 1.587224 \\ 12 & 6 & 1.563611 & .413960 & .084445 \\ 13 & 1 & -.627840 & 3.041755 & -.516891 \\ 14 & 6 & 1.183306 & -1.727833 & .342058 \\ 15 & 1 & 1.984598 & -2.176383 & .937825 \\ 16 & 1 & .456283 & -2.505485 & .092690 \\ 17 & 1 & 2.740232 & -1.081897 & -1.183698 \\ 18 & 1 & 1.042454 & -.850527 & -1.677001 \\ 19 & 1 & .870145 & -.338716 & 2.084915 \\ 20 & 1 & 2.524070 & .592447 & .587251 \\ 21 & 7 & -1.445157 & -.881292 & -.663084 \\ 22 & 7 & -2.428621 & -1.478981 & -.626689\end{array}$

CASPT2 $(12,12) / 6-31 G * / / U B 3 L Y P / 6-31 G$ *

Reference energy: $\quad-418.8267027190$

Total energy: $\quad-420.0494791487$ 


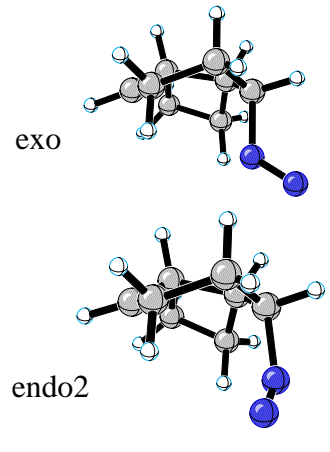

twist-boat diazenyl diradical

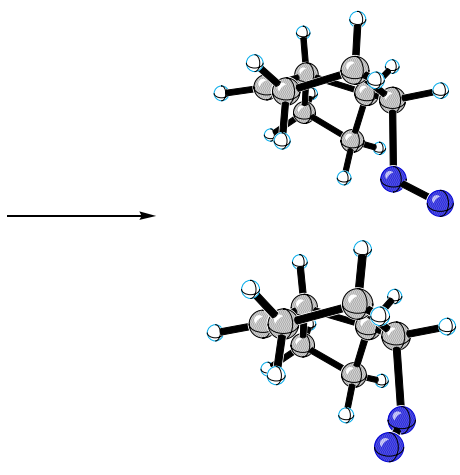

7-TS(C-II)

Intermediate from 7: endo but diazenyl diradical, twist-boat, exo $\mathrm{N}=\mathrm{N}$

UB3LYP/6-31G(d)

$\mathrm{E}(\mathrm{UB}+\mathrm{HF}-\mathrm{LYP})=-421.436011735$

Sum of electronic and zero-point Energies= $\quad-421.248674$

Sum of electronic and thermal Energies= $\quad-421.239042$

Sum of electronic and thermal Enthalpies= $\quad-421.238098$

Sum of electronic and thermal Free Energies= $\quad-421.284770$

$\begin{array}{cccr} & \mathrm{KCAL} / \mathrm{MOL} & \mathrm{CAL} / \mathrm{MOL}-\mathrm{KELVIN} & \mathrm{CAL} / \mathrm{MOL}-\mathrm{KELVIN} \\ \text { TOTAL } & 123.600 & 36.396 & 98.230\end{array}$

$\begin{array}{rrrrr}1 & 6 & -.966444 & -.291207 & .737282 \\ 2 & 6 & -1.272729 & 1.212083 & .735315 \\ 3 & 6 & -.611182 & 1.932174 & -.455482 \\ 4 & 1 & -2.355971 & 1.377102 & .727369 \\ 5 & 1 & -.895481 & 1.621443 & 1.682355 \\ 6 & 6 & 2.364562 & -.557350 & -.370450 \\ 7 & 6 & .805821 & 1.498461 & -.676187 \\ 8 & 1 & -1.203579 & 1.743641 & -1.364985 \\ 9 & 6 & .546767 & -.532755 & .860114 \\ 10 & 1 & 1.369744 & 1.934697 & -1.498370 \\ 11 & 1 & -1.518605 & -.805800 & 1.530847 \\ 12 & 6 & 1.490127 & .570774 & .263884 \\ 13 & 1 & -.672789 & 3.022205 & -.295045 \\ 14 & 6 & 1.265759 & -1.597156 & -.020259 \\ 15 & 1 & 1.594154 & -2.504052 & .496695 \\ 16 & 1 & .672950 & -1.889451 & -.890173 \\ 17 & 1 & 3.301384 & -.719129 & .171969 \\ 18 & 1 & 2.593651 & -.439284 & -1.434067 \\ 19 & 1 & .764934 & -.688772 & 1.922594 \\ 20 & 1 & 2.030221 & 1.133942 & 1.046313 \\ 21 & 7 & -1.499581 & -.859531 & -.592583 \\ 22 & 7 & -2.417091 & -1.597137 & -.627530\end{array}$

CASPT2 $(12,12) / 6-31 G * / / U B 3 L Y P / 6-31 G$ *

Reference energy: $\quad-418.8191640083$

Total energy: $\quad-420.0497186669$ 
$\operatorname{CASPT2}(6,6) / 6-31 G * / / U B 3 L Y P / 6-31 G *$

Reference energy: $\quad-418.7694054170$

Total energy: $\quad-420.0479886187$

Intermediate from 7: endo but diazenyl diradical, twist boat, endo2 $\mathrm{N}=\mathrm{N}$

\begin{tabular}{|c|c|c|c|c|c|c|}
\hline \multicolumn{7}{|c|}{ UB3LYP/6 - 31G(d) } \\
\hline $\mathrm{E}(\mathrm{UB}+\mathrm{H}$ & $F-L Y P)=-L$ & 421.435885988 & & & & \\
\hline Sum of & electronic & and zero-point & fergies= & & -42 & 248514 \\
\hline Sum of & electronic & and thermal Er & gies= & & -42 & 239065 \\
\hline Sum of & electronic & and thermal $\mathrm{Er}$ & alpies= & & -42 & 238121 \\
\hline Sum of & electronic & and thermal $\mathrm{Fr}$ & Energies= & & -42 & 283343 \\
\hline & & $\mathrm{E}$ (Thermal) & & & & $\mathrm{S}$ \\
\hline & & $\mathrm{KCAL} / \mathrm{MOL}$ & $\mathrm{CAL} / \mathrm{MOL}$ & ELVIN & & /MOL-KELVIN \\
\hline TOTAL & & 123.507 & & 369 & & 95.177 \\
\hline 1 & 6 & & -0.826507 & -0.54 & 3651 & 0.856372 \\
\hline 2 & 6 & & -1.415504 & 0.86 & 9411 & $\odot .850692$ \\
\hline 3 & 6 & & -0.961394 & 1.68 & 0227 & -0.375309 \\
\hline 4 & 1 & & -2.508913 & 0.82 & 1244 & 0.887010 \\
\hline 5 & 1 & & -1.082100 & 1.36 & 2563 & 1.773753 \\
\hline 6 & 6 & & 2.448512 & -0.15 & 4533 & -0.431327 \\
\hline 7 & 6 & & 0.501236 & 1.53 & 5496 & -0.653390 \\
\hline 8 & 1 & & -1.548320 & 1.35 & 5347 & -1.250554 \\
\hline 9 & 6 & & 0.710642 & -0.51 & 9743 & 0.844129 \\
\hline 10 & 1 & & $\odot .939913$ & 2.09 & 4766 & -1.477815 \\
\hline 11 & 1 & & -1.189547 & -1.11 & 1958 & 1.719489 \\
\hline 12 & 6 & & 1.391984 & 0.76 & 5871 & $\odot .255735$ \\
\hline 13 & 1 & & -1.234550 & 2.73 & 9339 & -0.230133 \\
\hline 14 & 6 & & 1.526698 & -1.37 & 3561 & -0.169415 \\
\hline 15 & 1 & & 1.992459 & -2.28 & 4021 & 0.220057 \\
\hline 16 & 1 & & $\odot .931838$ & -1.63 & 8410 & -1.046960 \\
\hline 17 & 1 & & 3.384107 & -0.20 & 0842 & 0.135648 \\
\hline 18 & 1 & & 2.682900 & 0.07 & 1346 & -1.476375 \\
\hline 19 & 1 & & 1.070002 & -0.69 & 6762 & 1.864326 \\
\hline 20 & 1 & & 1.825368 & 1.42 & 2287 & 1.031769 \\
\hline 21 & 7 & & -1.369570 & -1.40 & 8488 & -0.319442 \\
\hline 22 & 7 & & -2.275739 & -1.09 & ๑37० & $-\odot .997007$ \\
\hline
\end{tabular}

Transition structure 7-TS(C-II): cleave 2nd CN bond, twist-boat, exo N=N

UB3LYP/6-31G(d)

$E(U B+H F-L Y P)=-421.433154865$

Sum of electronic and zero-point Energies= $\quad-421.247960$

Sum of electronic and thermal Energies= $\quad-421.238352$

Sum of electronic and thermal Enthalpies= $\quad-421.237408$

Sum of electronic and thermal Free Energies= $\quad-421.284025$

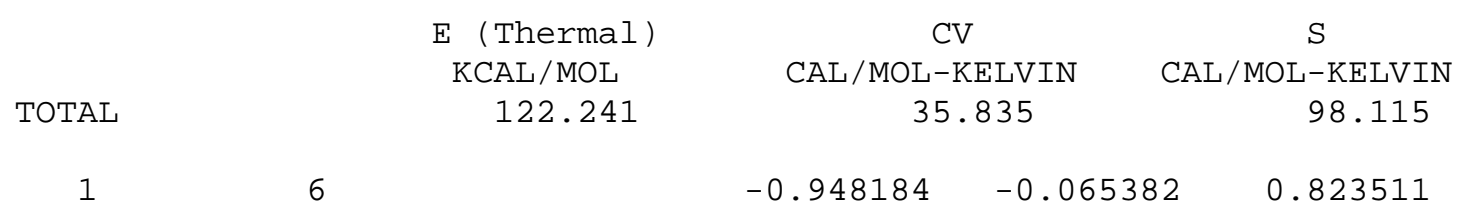




$\begin{array}{rrrrr}2 & 6 & -1.041072 & 1.438667 & 0.655854 \\ 3 & 6 & -0.238969 & 1.934821 & -0.560151 \\ 4 & 1 & -2.090242 & 1.746232 & 0.574212 \\ 5 & 1 & -0.648896 & 1.906280 & 1.572405 \\ 6 & 6 & 2.297563 & -0.913343 & -0.313828 \\ 7 & 6 & 1.152628 & 1.384876 & -0.597465 \\ 8 & 1 & -0.785278 & 1.647735 & -1.475762 \\ 9 & 6 & 0.466930 & -0.613239 & 0.842589 \\ 10 & 1 & 1.857559 & 1.784681 & -1.324241 \\ 11 & 1 & -1.588674 & -0.457098 & 1.614866 \\ 12 & 6 & 1.616541 & 0.330069 & 0.344274 \\ 13 & 1 & -0.224294 & 3.036113 & -0.568361 \\ 14 & 6 & 0.958902 & -1.682297 & -0.185771 \\ 15 & 1 & 0.999077 & -2.718070 & 0.165049 \\ 16 & 1 & 0.378434 & -1.645154 & -1.110138 \\ 17 & 1 & 3.085415 & -1.331031 & 0.321930 \\ 18 & 1 & 2.696234 & -0.775177 & -1.323590 \\ 19 & 1 & 0.689840 & -0.963297 & 1.858592 \\ 20 & 1 & 2.210993 & 0.768878 & 1.164764 \\ 21 & 7 & -1.809687 & -0.696727 & -0.617639 \\ 22 & 7 & -2.785485 & -1.286862 & -0.457191\end{array}$

CASPT2 $(12,12) / 6-31 G * / / U B 3 L Y P / 6-31 G$ *

Reference energy: $\quad-418.8233776589$

Total energy: $\quad-420.0451603236$

CASPT2 $(6,6) / 6-31 G * / / U B 3 L Y P / 6-31 G *$

Reference energy: $\quad-418.7737518276$

Total energy: $\quad-420.0435597952$

Transition structure 7-TS(C-II): cleave 2nd CN, twist-boat, endo2 $\mathrm{N}=\mathrm{N}$

UB3LYP/6-31G(d)

$\mathrm{E}(\mathrm{UB}+\mathrm{HF}-\mathrm{LYP})=-421.434066296$

Sum of electronic and zero-point Energies= $\quad-421.248657$

Sum of electronic and thermal Energies= $\quad-421.239222$

Sum of electronic and thermal Enthalpies= $\quad-421.238278$

Sum of electronic and thermal Free Energies= $\quad-421.283829$

$\begin{array}{cr} & \mathrm{KCAL} / \mathrm{MOL} \\ \text { TOTAL } & 122.267\end{array}$

E (Thermal)
CV

CAL/MOL-KELVIN 35.644
$S$

CAL/MOL-KELVIN

95.872

$\begin{array}{rrrrr}1 & 6 & -0.798814 & -0.426306 & 0.946532 \\ 2 & 6 & -1.351728 & 0.980675 & 0.832075 \\ 3 & 6 & -0.846605 & 1.711616 & -0.424557 \\ 4 & 1 & -2.447076 & 0.956024 & 0.836323 \\ 5 & 1 & -1.043202 & 1.530518 & 1.734117 \\ 6 & 6 & 2.446924 & -0.294790 & -0.446387 \\ 7 & 6 & 0.614500 & 1.509750 & -0.670862 \\ 8 & 1 & -1.430077 & 1.356519 & -1.291014 \\ 9 & 6 & 0.712363 & -0.522018 & 0.864238 \\ 10 & 1 & 1.086336 & 2.045161 & -1.493103 \\ 11 & 1 & -1.221470 & -0.984333 & 1.784511 \\ 12 & 6 & 1.466640 & 0.704614 & 0.243855 \\ 13 & 1 & -1.088807 & 2.784662 & -0.344730\end{array}$




$\begin{array}{rrrrr}14 & 6 & 1.431794 & -1.433812 & -0.179804 \\ 15 & 1 & 1.822169 & -2.387675 & 0.187838 \\ 16 & 1 & 0.795516 & -1.627903 & -1.046110 \\ 17 & 1 & 3.374656 & -0.415691 & 0.122799 \\ 18 & 1 & 2.699835 & -0.088988 & -1.491201 \\ 19 & 1 & 1.109458 & -0.735682 & 1.865356 \\ 20 & 1 & 1.953725 & 1.347183 & 0.999005 \\ 21 & 7 & -1.518891 & -1.417857 & -0.338290 \\ 22 & 7 & -2.432752 & -1.033310 & -0.926615\end{array}$



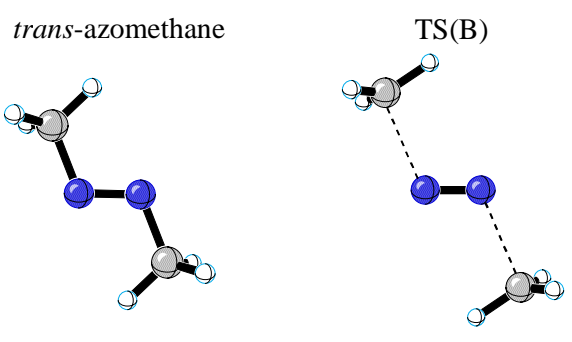

methyl diazenyl

TS(C-II)

\section{trans-azomethane: reactant}

B3LYP/ $6-31 G(d)$
E(RB+HF-LYP) $=$
Sum of electronic
Sum of electronic
Sum of electronic
Sum of electronic


TOTAL

$-189.275397711$

onic and zero-point Energies=

$-189.190451$

$-189.185330$

$-189.184386$

$-189.217452$

E (Thermal) $\mathrm{KCAL} / \mathrm{MOL}$ 56.518
CV CAL/MOL-KELVIN 16.284
$S$ CAL/MOL-KELVIN 69.593

B3LYP/6-311G (d, p)

$E(R B+H F-L Y P)=-189.328010822$

Sum of electronic and zero-point Energies= -189.243983

Sum of electronic and thermal Energies= -189.238834

Sum of electronic and thermal Enthalpies= $\quad-189.237890$

Sum of electronic and thermal Free Energies= $\quad-189.271010$

$\begin{array}{lr} & \mathrm{KCAL} / \mathrm{MOL} \\ \text { TOTAL } & 55.959\end{array}$

$\begin{array}{rrr}0.426881 & 0.452630 & -0.000530 \\ -0.426742 & -0.452376 & -0.000531 \\ 1.798064 & -0.069327 & 0.000349 \\ 2.310654 & 0.330233 & 0.883081 \\ 1.828533 & -1.164811 & 0.000893 \\ 2.311328 & 0.329395 & -0.882358 \\ -1.798160 & 0.069171 & 0.000352 \\ -1.828969 & 1.164631 & 0.00 \odot 978 \\ -2.311283 & -0.329652 & -0.882392 \\ -2.310663 & -0.330639 & 0.883017\end{array}$

$\begin{array}{rl}1 & 7 \\ 2 & 7 \\ 3 & 6 \\ 4 & 1 \\ 5 & 1 \\ 6 & 1 \\ 7 & 6 \\ 8 & 1 \\ 9 & 1 \\ 10 & 1\end{array}$

-0.426311
0.426327
-1.797753
-2.307757
-1.824119
-2.307801
1.797742
1.824072
2.307796
2.307758

CV

CAL/MOL-KELVIN

16.405
$S$

CAL/MOL-KELVIN

69.706 


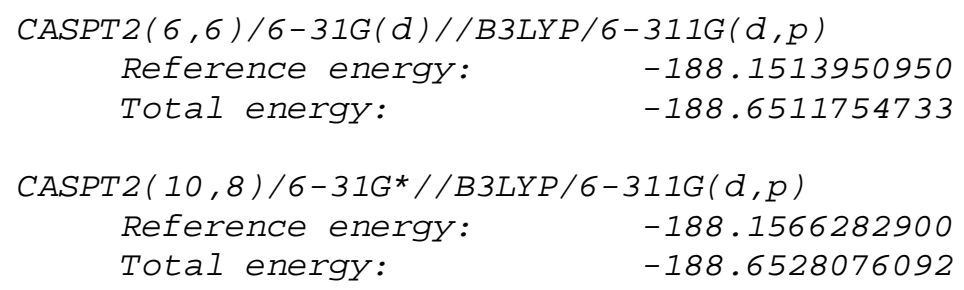

\begin{tabular}{|c|c|c|c|c|c|c|}
\hline \multirow{2}{*}{\multicolumn{7}{|c|}{$\operatorname{CASSCF}(4,4) / 6-31 G^{*}$}} \\
\hline & & & & & & \\
\hline Sum of & electronic & and zero-poin & nergies= & & -188 & 017253 \\
\hline Sum of & electronic & and thermal $\mathrm{Er}$ & gies $=$ & & -188 & 012175 \\
\hline Sum of & electronic & and thermal $\mathrm{Er}$ & alpies= & & -188 & 011231 \\
\hline Sum of & electronic & and thermal $\mathrm{Fr}$ & Energies= & & -188 & 043614 \\
\hline & & E (Thermal) & c & & & $\mathrm{S}$ \\
\hline & & KCAL/MOL & CAL/MOL - & ELVIN & & /MOL-KELVIN \\
\hline TOTAL & & 59.920 & & 798 & & 68.156 \\
\hline 1 & 7 & & $\odot .00000 \odot$ & 0.60 & 5507 & $\odot .000 \odot \odot \odot$ \\
\hline 2 & 7 & & $\odot .000000$ & -0.60 & 5507 & $\odot .000 \odot \odot \odot$ \\
\hline 3 & 6 & & 1.368594 & 1.18 & 6707 & $\odot .00000 \odot$ \\
\hline 4 & 1 & & 1.460403 & 1.81 & 5347 & 0.877792 \\
\hline 5 & 1 & & 2.129901 & 0.41 & 7043 & $\odot .00000 \odot$ \\
\hline 6 & 1 & & 1.460403 & 1.81 & 5347 & $-\odot .877792$ \\
\hline 7 & 6 & & -1.368594 & -1.18 & 6707 & $\odot .00 \odot \odot \odot \odot$ \\
\hline 8 & 1 & & -2.129901 & -0.41 & 7043 & $\odot .00000 \odot$ \\
\hline 9 & 1 & & -1.460403 & -1.81 & 5347 & -0.877792 \\
\hline 10 & 1 & & -1.460403 & -1.81 & 5347 & 0.877792 \\
\hline
\end{tabular}

CASPT2 $(4,4) / 6-31 G(d) / / \operatorname{CASSCF}(4,4) / 6-31 G^{*}$

Reference energy: $\quad-188.1062355334$

Total energy: $\quad-188.6488018315$

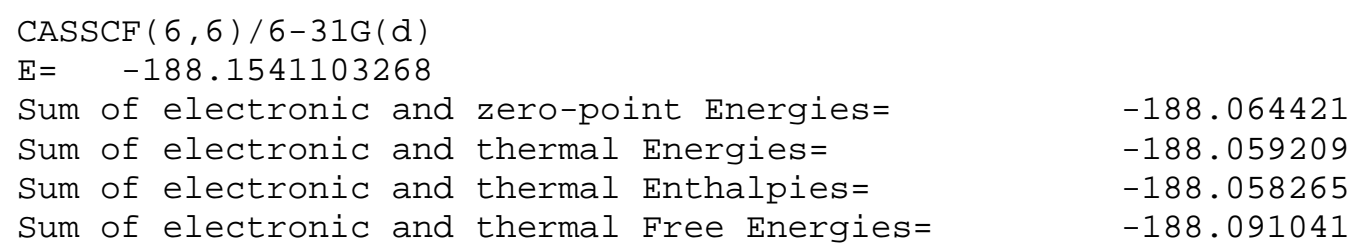

E (Thermal) $\mathrm{KCAL} / \mathrm{MOL}$

TOTAL $\quad 59.551$
CV CAL/MOL-KELVIN 16.008
$S$

CAL/MOL-KELVIN

68.983

$\begin{array}{ll}1 & 7 \\ 2 & 7 \\ 3 & 6 \\ 4 & 1 \\ 5 & 1 \\ 6 & 1 \\ 7 & 6\end{array}$

$\begin{array}{rrr}0.0000 \odot \odot & 0.617468 & 0.000 \odot \odot \odot \\ 0.000 \odot \odot \odot & -0.617468 & 0.00 \odot \odot \odot \odot \\ 1.383203 & 1.184209 & 0.00 \odot \odot \odot \odot \\ 1.480992 & 1.809619 & 0.878689 \\ 2.134698 & 0.405237 & 0.00 \odot \odot \odot \odot \\ 1.480992 & 1.809619 & -0.878689 \\ -1.383203 & -1.184209 & 0.00 \odot \odot \odot \odot\end{array}$




$\begin{array}{rrrrr}8 & 1 & -2.134698 & -0.405237 & 0.000000 \\ 9 & 1 & -1.480992 & -1.809619 & -0.878689 \\ 10 & 1 & -1.480992 & -1.809619 & 0.878689\end{array}$
$\operatorname{CASPT2}(6,6) / 6-31 G(d) / / \operatorname{CASSCF}(6,6) / 6-31 G(d)$
Reference energy: $\quad-188.1526393102$
Total energy: $\quad-188.6503028710$

\begin{tabular}{|c|c|c|c|c|c|c|}
\hline \multicolumn{7}{|c|}{$\operatorname{CASSCF}(10,8) / 6-31 G^{*}$} \\
\hline$E=$ & -188.1595988 & $\odot 47$ & & & & \\
\hline Sum 0 & of electronic & and zero-point & nergies= & & -188 & 070088 \\
\hline Sum 0 & of electronic & and thermal En & gies= & & -188 & 064856 \\
\hline Sum 0 & of electronic & and thermal En & alpies= & & -188 & 063912 \\
\hline Sum 0 & of electronic & and thermal $\mathrm{Fr}$ & Energies= & & -188 & 096732 \\
\hline & & E (Thermal) & & & & $\mathrm{S}$ \\
\hline & & KCAL/MOL & $\mathrm{CAL} / \mathrm{MOL}$ & ELVIN & & /MOL-KELVIN \\
\hline TOTAL & & 59.452 & & 083 & & 69.076 \\
\hline 1 & 7 & & $\odot . \odot \odot \odot \odot \odot \odot$ & 0.61 & 6450 & $\odot .00 \odot \odot \odot \odot$ \\
\hline 2 & 7 & & $\odot .00000 \odot$ & -0.61 & 6450 & $\odot .00000 \odot$ \\
\hline 3 & 6 & & 1.388004 & 1.18 & 2982 & $\odot .00000 \odot$ \\
\hline 4 & 1 & & 1.486457 & 1.80 & 7938 & $\odot .878951$ \\
\hline 5 & 1 & & 2.137874 & 0.40 & 2349 & $\odot .00 \odot \odot \odot \odot$ \\
\hline 6 & 1 & & 1.486457 & 1.80 & 7938 & $-\odot .878951$ \\
\hline 7 & 6 & & -1.388004 & -1.18 & 2982 & $\odot . \odot \odot \odot \odot \odot \odot$ \\
\hline 8 & 1 & & -2.137874 & -0.40 & 2349 & $\odot .0 \odot \odot \odot \odot \odot$ \\
\hline 9 & 1 & & -1.486457 & -1.80 & 7938 & -0.878951 \\
\hline 10 & 1 & & -1.486457 & -1.80 & 7938 & 0.878951 \\
\hline
\end{tabular}

$\operatorname{CASPT2}(10,8) / / 6-31 G * / / \operatorname{CASSCF}(10,8) / 6-31 G^{*}$

Reference energy: $\quad-188.1581030217$

Total energy: $\quad-188.6517335786$

trans-azomethane: TS(B), concerted cleavage of both CN bonds

B3LYP/6-311G (d, p)

$\mathrm{E}(\mathrm{UB}+\mathrm{HF}-\mathrm{LYP})=-189.236723539$

Sum of electronic and zero-point Energies= $\quad-189.164342$

Sum of electronic and thermal Energies= $\quad-189.156454$

Sum of electronic and thermal Enthalpies= -189.155509

Sum of electronic and thermal Free Energies= $\quad-189.197657$

$\begin{array}{cccc} & \text { KCAL/MOL } & \text { CAL/MOL-KELVIN } & \text { CAL/MOL-KELVIN } \\ \text { TOTAL } & 50.370 & 22.861 & 88.707\end{array}$

$\begin{array}{rrrrr}1 & 7 & -0.334201 & -0.457435 & -0.000057 \\ 2 & 7 & 0.334178 & 0.457454 & -0.000056 \\ 3 & 6 & -2.398048 & 0.051906 & 0.000032 \\ 4 & 1 & -2.676334 & -0.436960 & 0.924359 \\ 5 & 1 & -2.357794 & 1.133574 & -0.002216 \\ 6 & 1 & -2.677408 & -0.440785 & -0.921933\end{array}$




$\begin{array}{rrrrr}7 & 6 & 2.398071 & -0.051920 & 0.000033 \\ 8 & 1 & 2.676439 & 0.437273 & 0.924161 \\ 9 & 1 & 2.357790 & -1.133588 & -0.001834 \\ 10 & 1 & 2.677337 & 0.440442 & -0.922136\end{array}$

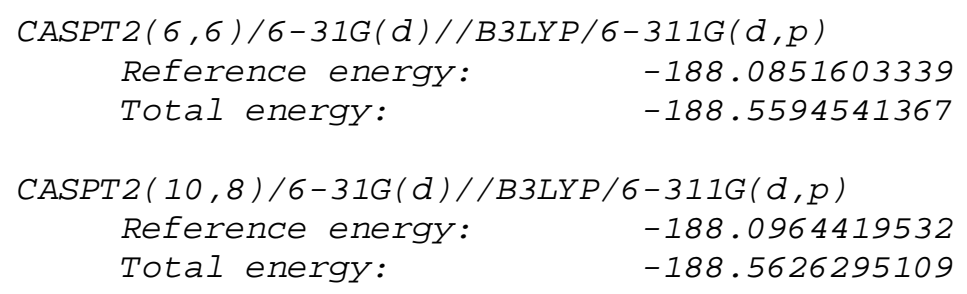

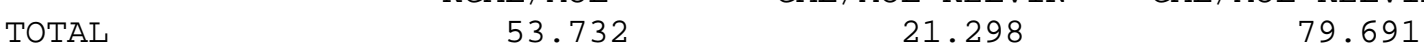

\section{$\operatorname{CASSCF}(6,6) / 6-31 \mathrm{G}(\mathrm{d})$}

$E=\quad-188.0843086389$

Sum of electronic and zero-point Energies=

Sum of electronic and thermal Energies=

Sum of electronic and thermal Enthalpies=

Sum of electronic and thermal Free Energies=

E (Thermal) $\mathrm{KCAL} / \mathrm{MOL}$

TOTAL 53.700
CV CAL/MOL-KELVIN 21.007
$-188.005726$

$-187.998732$

$-187.997788$

$-188.035398$

KELVI 


$\begin{array}{rrrrr}4 & 1 & 1.958877 & 1.806963 & 0.908149 \\ 5 & 1 & 2.544475 & 0.340570 & 0.0000 \odot \odot \\ 6 & 1 & 1.958877 & 1.806963 & -0.908149 \\ 7 & 6 & -1.939259 & -1.230564 & 0.000000 \\ 8 & 1 & -1.958877 & -1.806963 & 0.908149 \\ 9 & 1 & -2.544475 & -0.340570 & 0.000 \odot \odot \odot \\ 10 & 1 & -1.958877 & -1.806963 & -0.908149\end{array}$

$\operatorname{CASPT} 2(6,6) / 6-31 G(d) / / \operatorname{CASSCF}(6,6) / 6-31 G(d)$

Reference energy: $\quad-188.0830843524$

Total energy: $\quad-188.5626894239$

$\begin{array}{ll}\text { CASSCF }(10,8) / 6-31 G(d) & \\ \text { E= -188.0951424932 } & -188.016533 \\ \text { Sum of electronic and zero-point Energies= } & -188.009492 \\ \text { Sum of electronic and thermal Energies= } & -188.008548 \\ \text { Sum of electronic and thermal Enthalpies= } & -188.046320 \\ \text { Sum of electronic and thermal Free Energies= } & \end{array}$

$\begin{array}{rr}\mathrm{KCAL} / \mathrm{MOL} \\ \text { TOTAL } & 53.746\end{array}$

CV S

\begin{tabular}{|c|c|c|c|c|}
\hline ГOTAL & & 53.746 & 006 & 79.497 \\
\hline 1 & 7 & $\odot . \odot \odot \odot \odot \odot \odot$ & 0.574144 & $\odot .0 \odot \odot \odot \odot \odot$ \\
\hline 2 & 7 & $\odot . ๑ \odot \odot \odot \odot \odot$ & -0.574144 & $\odot .0 \odot \odot \odot \odot \odot$ \\
\hline 3 & 6 & 1.926233 & 1.243811 & $\odot .0 \odot \odot \odot \odot \odot$ \\
\hline 4 & 1 & 1.945297 & 1.821212 & $\odot .907733$ \\
\hline 5 & 1 & 2.539158 & ๑. 358887 & $\odot . \odot \odot \odot \odot \odot \odot$ \\
\hline 6 & 1 & 1.945297 & 1.821212 & -0.907733 \\
\hline 7 & 6 & -1.926233 & -1.243811 & $\odot .0000 \odot \odot$ \\
\hline 8 & 1 & -1.945297 & -1.821212 & $\odot .907733$ \\
\hline 9 & 1 & -2.539158 & -0.358887 & ๑. . \\
\hline 10 & 1 & -1.945297 & -1.821212 & -0.907733 \\
\hline
\end{tabular}

$\begin{array}{cr}\text { CASPT2 }(10,8) / 6-31 G(d) / / \operatorname{CASSCF}(10,8) / 6-31 G(d) \\ \text { Reference energy: } & -188.0939197809 \\ \text { Total energy: } & -188.5659012155\end{array}$

\section{intermediate from trans-azomethane: methyl diazenyl radical}

$B 3 L Y P / 6-31 G(d)$

$\mathrm{E}(\mathrm{UB}+\mathrm{HF}-\mathrm{LYP})=-149.348310006$

Sum of electronic and zero-point Energies= -149.304454

Sum of electronic and thermal Energies= -149.300572

Sum of electronic and thermal Enthalpies= $-\mathbf{- 1 4 9 . 2 9 9 6 2 8}$

Sum of electronic and thermal Free Energies= $-\mathbf{1 4 9 . 3 2 9 8 1 3}$

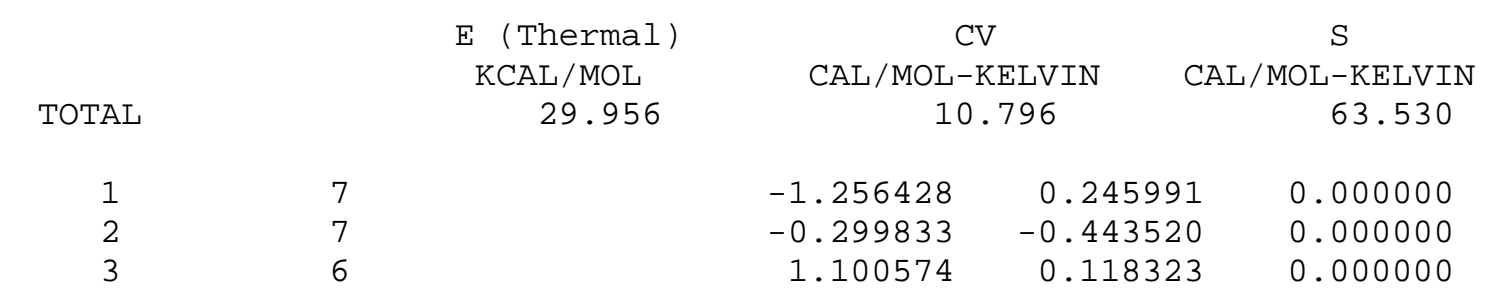




$\begin{array}{rrrrr}4 & 1 & 1.085202 & 1.211646 & -0.000016 \\ 5 & 1 & 1.602586 & -0.269425 & 0.889286 \\ 6 & 1 & 1.602598 & -0.269453 & -0.889267\end{array}$

B3LYP/ 6-311G(d, p)

$E(U B+H F-$ LYP $)=-149.389394903$

Sum of electronic and zero-point Energies= $\quad-149.346091$

Sum of electronic and thermal Energies= -149.342175

Sum of electronic and thermal Enthalpies= -149.341231

Sum of electronic and thermal Free Energies= $\quad-149.371495$

$\begin{array}{rr} & \mathrm{KCAL} / \mathrm{MOL} \\ \text { TOTAL } & 29.631\end{array}$

$\begin{array}{ccc}\text { (Thermal) } & \text { CV } & \mathrm{S} \\ \mathrm{KCAL} / \mathrm{MOL} & \mathrm{CAL} / \mathrm{MOL}-\mathrm{KELVIN} & \mathrm{CAL} / \mathrm{MOL}-\mathrm{KELVIN}\end{array}$

$10.917 \quad 63.696$

$\begin{array}{rrrrr}1 & 7 & -1.254156 & 0.244270 & 0.000000 \\ 2 & 7 & -0.304355 & -0.438562 & 0.00000 \odot \\ 3 & 6 & 1.103415 & 0.116678 & 0.000000 \\ 4 & 1 & 1.088266 & 1.207805 & -0.000003 \\ 5 & 1 & 1.600409 & -0.273914 & 0.887560 \\ 6 & 1 & 1.600412 & -0.273919 & -0.887556\end{array}$

CASPT2 $(5,5) / 6-31 G(d) / / B 3 L Y P / 6-311 G(d, p)$

Reference energy: $\quad-148.5181734984$

Total energy: $\quad-148.8911525062$

CASPT2 $(9,7) / 6-31 G * / / B 3 L Y P / 6-311 G(d, p)$

Reference energy: $\quad-148.5346220224$

Total energy: $\quad-148.8944386503$

$\operatorname{CASSCF}(3,3) / 6-31 G(d)$

$\mathrm{E}=\quad-148.4733564542$

Sum of electronic and zero-point Energies= $\quad-148.426780$

Sum of electronic and thermal Energies= $\quad-148.422957$

Sum of electronic and thermal Enthalpies= $\quad-148.422013$

Sum of electronic and thermal Free Energies= $\quad-148.452097$

$\begin{array}{rr}\mathrm{KCAL} / \mathrm{MOL} \\ \text { TOTAL } & 31.626\end{array}$

$\mathrm{E}$ (Thermal) CV S

KCAL/MOL CAL/MOL-KELVIN CAL/MOL-KELVIN

$10.493 \quad 63.317$

$\begin{array}{rrrrr}1 & 7 & 1.156944 & 0.468922 & 0.000 \odot \odot \odot \odot \\ 2 & 7 & 0.000 \odot \odot \odot & 0.566438 & 0.00 \odot \odot \odot \odot \\ 3 & 6 & -0.831739 & -0.714866 & 0.000000 \\ 4 & 1 & -0.200013 & -1.592274 & 0.00 \odot \odot \odot \odot \\ 5 & 1 & -1.454078 & -0.683023 & 0.882365 \\ 6 & 1 & -1.454078 & -0.683023 & -0.882365\end{array}$

$\operatorname{CASPT2}(3,3) / 6-31 G(d) / / \operatorname{CASSCF}(3,3) / 6-31 G(d)$

Reference energy: $\quad-148.4722416289$

Total energy: $\quad-148.8939081782$

$\operatorname{CASSCF}(5,5) / 6-31 G(d)$ 
$E=\quad-148.5212724075$

Sum of electronic and zero-point Energies=

Sum of electronic and thermal Energies=

Sum of electronic and thermal Enthalpies=

$-148.476284$

Sum of electronic and thermal Free Energies=

$-148.472170$

$-148.471226$

E (Thermal)

CV

$-148.501935$

TOTAL KCAL/MOL 30.812

CAL/MOL - KELVIN

$\mathrm{S}$

$\begin{array}{ll}1 & 7 \\ 2 & 7 \\ 3 & 6 \\ 4 & 1 \\ 5 & 1 \\ 6 & 1\end{array}$

11.402

CAL/MOL - KELVIN

64.634

$\begin{array}{cc}\text { CASPT2 }(5,5) / 6-31 G(d) / / \operatorname{CASSCF}(5,5) / 6-31 G(d) \\ \text { Reference energy: } & -148.5200914975 \\ \text { Total energy: } & -148.8891376458\end{array}$

$\operatorname{CASSCF}(9,7) / 6-31 \mathrm{G}$ *

$\mathrm{E}=\quad-148.5367606776$

Sum of electronic and zero-point Energies=

Sum of electronic and thermal Energies=

Sum of electronic and thermal Enthalpies=

$-148.491378$

1.165389

0.484443

$\odot .0 \odot \odot \odot \odot \odot$

0.000000

0.595410

$-0.843375$

$-0.753019$

๑. . $00000 \odot$

$-0.185785$

$-1.610138$

०. . 000000

$-1.455842$

$-0.715360$

0.000000

$-0.715360$

0.887407

$-\odot .887407$

$-148.487310$

$-148.486366$

$-148.517059$

Sum of electronic and thermal Free Energies=

\section{$S$}

$\begin{array}{rr}\text { TOTAL } & 31.031\end{array}$

E (Thermal)
CAL/MOL - KELVIN

11.195
$\mathrm{CAL} / \mathrm{MOL}-\mathrm{KELVIN}$

$\begin{array}{rrrrr}1 & 7 & 1.166622 & 0.520609 & 0.00000 \odot \\ 2 & 7 & 0.00000 \odot & 0.569209 & 0.00000 \odot \\ 3 & 6 & -0.843665 & -0.759273 & 0.00000 \odot \\ 4 & 1 & -0.186521 & -1.616412 & 0.00000 \odot \\ 5 & 1 & -1.458921 & -0.728341 & 0.88610 \odot \\ 6 & 1 & -1.458921 & -0.728341 & -0.886100\end{array}$

$\operatorname{CASPT2}(9,7) / 6-31 G(d) / / \operatorname{CASSCF}(9,7) / 6-31 G(d)$

Reference energy: $\quad-148.5355684067$

Total energy: $\quad-148.8933365066$

\section{TS of methyl diazenyl radical: TS(C-II), cleave CN bond}

$B 3 L Y P / 6-31 G(d)$

$\mathrm{E}(\mathrm{UB}+\mathrm{HF}-\mathrm{LYP})=-149.340476177$

Sum of electronic and zero-point Energies= $\quad-149.300045$

Sum of electronic and thermal Energies= -149.295905

Sum of electronic and thermal Enthalpies= -149.294961

Sum of electronic and thermal Free Energies= $\quad-149.326240$

E (Thermal)

CV

$S$

$\mathrm{KCAL} / \mathrm{MOL}$

CAL/MOL-KELVIN CAL/MOL-KELVIN 
TOTAL

$\begin{array}{ll}1 & 7 \\ 2 & 7 \\ 3 & 6 \\ 4 & 1 \\ 5 & 1 \\ 6 & 1\end{array}$

27.969

11.233

\begin{abstract}
$-1.303575$
$-0.464906$

1.296873

1.237777

1.680162

1.680193
\end{abstract}

๑. 301893

$-0.472133$

0.110796

1.196051

$-0.334526$

$-0.334619$

65.831

0.000000

0.000000

$\odot .000000$

$-0.000056$

$\odot .913909$

$-0.913851$

B3LYP/6-311G (d, p)

$\mathrm{E}(\mathrm{UB}+\mathrm{HF}-\mathrm{LYP})=-149.383587608$

Sum of electronic and zero-point Energies=

Sum of electronic and thermal Energies=

Sum of electronic and thermal Enthalpies=

$-149.343461$

Sum of electronic and thermal Free Energies=

$-149.339329$

$-149.338384$

$-149.369704$

E (Thermal) $\mathrm{KCAL} / \mathrm{MOL}$

TOTAL

$\begin{array}{ll}1 & 7 \\ 2 & 7 \\ 3 & 6 \\ 4 & 1 \\ 5 & 1 \\ 6 & 1\end{array}$

27.773
CV CAL/MOL-KELVIN 11.147
$S$ CAL/MOL - KELVIN 65.917

CASPT2 $(5,5) / 6-31 G(d) / / B 3 L Y P / 6-311 G(d, p)$ Reference energy: $-148.5241463838$

Total energy:

$-148.8814979752$

CASPT2 $(9,7) / / 6-31 G(d) / / B 3 L Y P / 6-311 G(d, p)$

Reference energy:

$-148.5385629804$

Total energy:

$-148.8859217834$

0.294448
-0.464935
0.110308
1.193987
-0.331225
-0.331205

$\odot .000000$

$\odot .000000$

$\odot .000000$

$-0.000012$

$-0.910268$

0.910281

$\operatorname{CASSCF}(3,3) / 6-31 G(d)$

$\mathrm{E}=-148.4691813305$

Sum of electronic and zero-point Energies=

Sum of electronic and thermal Energies=

$-148.425718$

Sum of electronic and thermal Enthalpies=

$-148.421794$

Sum of electronic and thermal Free Energies=

$-148.420850$

$-148.451490$

E (Thermal) $\mathrm{KCAL} / \mathrm{MOL}$

TOTAL 29.736
CV CAL/MOL-KELVIN 10.412
$S$

CAL/MOL-KELVIN 64.487

$\begin{array}{ll}1 & 7 \\ 2 & 7 \\ 3 & 6 \\ 4 & 1 \\ 5 & 1 \\ 6 & 1\end{array}$

1.133528
0.000000
-0.831234
-0.092956
-1.427166
-1.427166

0.592282

0.651553

$-0.887160$

$-1.672814$

$-0.855531$

$-0.855531$
0.000000

$\odot .00000 \odot$

$\odot .000000$

0.000000

$\odot .896753$

$-0.896753$ 
$\operatorname{CASPT2}(3,3) / 6-31 G(d) / / \operatorname{CASSCF}(3,3) / 6-31 G(d)$

Reference energy: $\quad-148.4680766113$

Total energy: $\quad-148.8860815047$

\begin{tabular}{|c|c|c|c|c|c|c|}
\hline \\
\hline \multicolumn{7}{|c|}{$\begin{array}{l}\operatorname{CASSCF}(5,5) / 6-31 G(d) \\
E=\quad-148.5211945139\end{array}$} \\
\hline Sum 0 & of electronic & and zero-poin & nergies= & & \multicolumn{2}{|c|}{-148.477507} \\
\hline Sum 0 & of electronic & and thermal Er & gies= & & \multicolumn{2}{|c|}{-148.473642} \\
\hline Sum 0 & of electronic & and thermal Er & alpies= & & \multicolumn{2}{|c|}{-148.472698} \\
\hline Sum 0 & of electronic & and thermal & Energies= & & \multicolumn{2}{|c|}{-148.503162} \\
\hline & & E (Thermal) & \multicolumn{2}{|c|}{ CV } & & $\begin{array}{c}\mathrm{S} \\
/ \mathrm{MOI}-\mathrm{KF} / \mathrm{VTN}\end{array}$ \\
\hline TOTAL & & 29.839 & \multicolumn{2}{|c|}{10.124} & & 64.116 \\
\hline 1 & 7 & & 1.158213 & 0.52 & 2602 & $\odot .00 \odot \odot \odot \odot$ \\
\hline 2 & 7 & & $\odot .00000 \odot$ & 0.61 & 3896 & $\odot .00000 \odot$ \\
\hline 3 & 6 & & -0.843035 & -0.80 & 3741 & $\odot .000000$ \\
\hline 4 & 1 & & -0.153140 & -1.63 & 3770 & ๑.०००००० \\
\hline 5 & 1 & & -1.448071 & -0.76 & 7133 & 0.891471 \\
\hline 6 & 1 & & -1.448071 & -0.76 & 7133 & $-\odot .891471$ \\
\hline
\end{tabular}

$\operatorname{CASPT2}(5,5) / 6-31 G(d) / / \operatorname{CASSCF}(5,5) / 6-31 G(d)$

Reference energy: $\quad-148.5200100671$

Total energy: -148.8864806056

\section{$\operatorname{CASSCF}(9,7) / 6-31 G(d)$}

$E=-148.5363296351$

Sum of electronic and zero-point Energies= $\quad-148.492785$

Sum of electronic and thermal Energies= $\quad-148.488849$

Sum of electronic and thermal Enthalpies= $\quad-148.487905$

Sum of electronic and thermal Free Energies= $\quad-148.518625$

E (Thermal)
KCAL/MOL
29.795

TOTAL

$\begin{array}{ll}1 & 7 \\ 2 & 7 \\ 3 & 6 \\ 4 & 1 \\ 5 & 1 \\ 6 & 1\end{array}$

CV CAL/MOL - KELVIN 10.270
$S$

CAL/MOL-KELVIN

64.655

$\operatorname{CASPT2}(9,7) / 6-31 G(d) / / \operatorname{CASSCF}(9,7) / 6-31 G(d)$

Reference energy: $\quad-148.5351469096$

Total energy: $\quad-148.8892836351$

$\odot .000000$

$\odot .000000$

$\odot .000000$

0.000000

$\odot .892663$

$-0.892663$ 


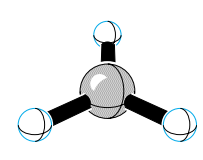

intermediate from trans-azomethane: methyl radical

$B 3 L Y P / 6-31 G(d)$

$E(U B+H F-L Y P)=-39.8382914868$

Sum of electronic and zero-point Energies= $-\mathbf{- 3 9 . 8 0 8 4 7 6}$

Sum of electronic and thermal Energies= -39.805374

Sum of electronic and thermal Enthalpies $=\quad-39.804430$

Sum of electronic and thermal Free Energies= $\quad-39.827264$

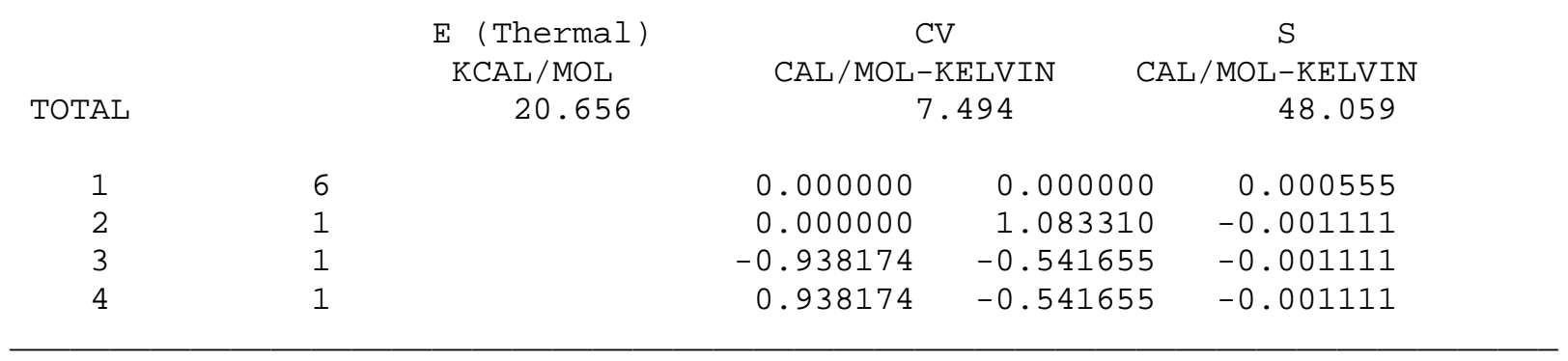

B3LYP/ 6-311G (d, p)

$E(U B+H F-L Y P)=-39.8537575454$

Sum of electronic and zero-point Energies= -39.824183

Sum of electronic and thermal Energies= $\quad-39.821115$

Sum of electronic and thermal Enthalpies= $\quad-39.820171$

Sum of electronic and thermal Free Energies= $\quad-39.842940$

$\begin{array}{cccc} & \mathrm{KCAL} / \mathrm{MOL} & \mathrm{CAL} / \mathrm{MOL}-\mathrm{KELVIN} & \mathrm{CAL} / \mathrm{MOL}-\mathrm{KELVIN} \\ \text { TOTAL } & 20.483 & 7.409 & 47.921\end{array}$

$$
\begin{array}{ccc}
\text { E (Thermal) } & \text { CV } & S \\
\text { KCAL/MOL } & \text { CAL/MOL-KELVIN } & \text { CAL/MOL-KELVIN } \\
20.483 & 7.409 & 47.921
\end{array}
$$

$\begin{array}{rrrrr}1 & 6 & 0.00 \odot \odot \odot \odot & 0.00 \odot \odot \odot \odot & 0.00 \odot \odot \odot ४ 4 \\ 2 & 1 & 0.000 \odot \odot \odot & 1.080488 & -0.00 \odot \odot \odot 8 \\ 3 & 1 & -0.935730 & -0.540244 & -0.00 \odot \odot \odot 8 \\ 4 & 1 & 0.935730 & -0.540244 & -0.00 \odot \odot \odot 8\end{array}$
CASPT2 $(1,1) / 6-31 G(d) / / B 3 L Y P / 6-311 G(d, p)$
Reference energy: $\quad-39.5542939258$
Total energy: $\quad-39.6727090388$

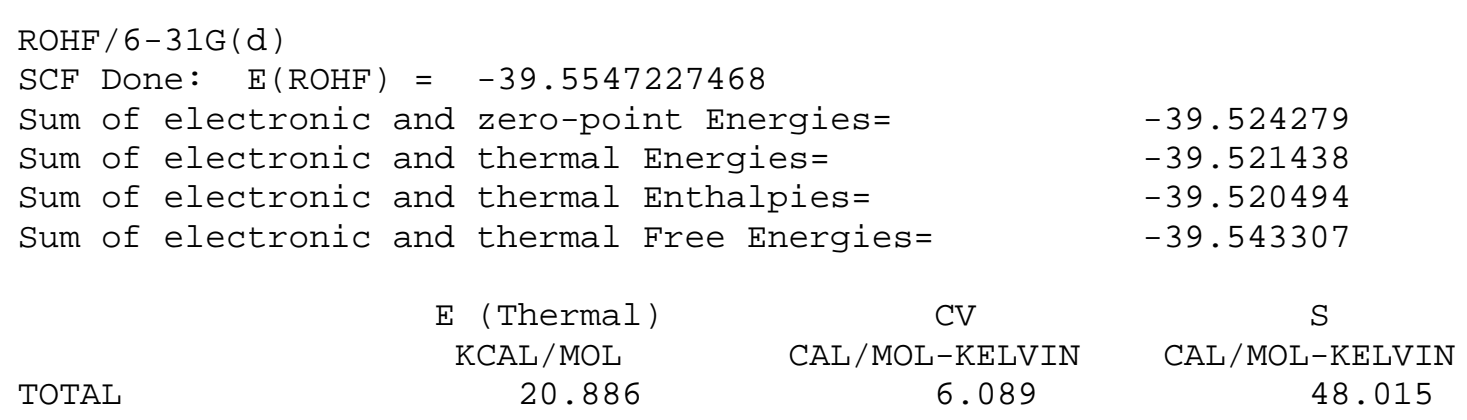




$\begin{array}{rrrrr}1 & 6 & 0.000 \odot \odot \odot & 0.0000 \odot \odot & 0.0000 \odot \odot \\ 2 & 1 & 0.00 \odot \odot \odot \odot & 0.000 \odot \odot \odot & -1.071125 \\ 3 & 1 & 0.000 \odot \odot \odot & 0.927622 & 0.535562 \\ 4 & 1 & 0.000 \odot \odot \odot & -0.927622 & 0.535562\end{array}$

CASPT2 $(1,1) / 6-31 G(d) / / R O H F / 6-31 G(d)$

Reference energy:

Total energy:
$-39.5544863837$

$-39.6725793004$ 

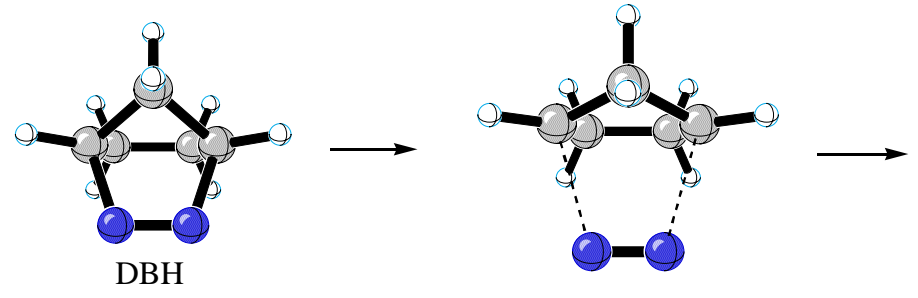

TS 2-bond cleavage

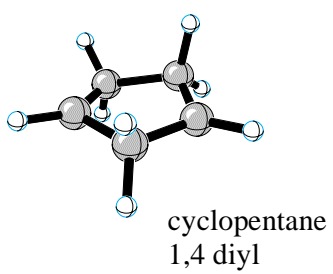

\section{Reactant: DBH}

RB3LYP/6-31G(d)

$E(R B+H F-L Y P)=-304.793450401$

Sum of electronic and zero-point Energies=

Sum of electronic and thermal Energies=

$-304.663481$

$-304.658484$

Sum of electronic and thermal Enthalpies=

$-304.657540$

Sum of electronic and thermal Free Energies=

$-304.691903$

$\begin{array}{cc} & \mathrm{KCAL} / \mathrm{MOL} \\ \text { TOTAL } & 84.693\end{array}$

$$
\text { E (Thermal) }
$$

$\begin{array}{rl}1 & 6 \\ 2 & 6 \\ 3 & 1 \\ 4 & 6 \\ 5 & 1 \\ 6 & 1 \\ 7 & 1 \\ 8 & 6 \\ 9 & 1 \\ 10 & 7 \\ 11 & 1 \\ 12 & 1 \\ 13 & 7 \\ 14 & 6 \\ 15 & 1\end{array}$

CV

CAL/MOL-KELVIN 20.452
$S$

CAL/MOL-KELVIN

72.325

CASPT2 $(6,6) / 6-31 G * / / B 3 L Y P / 6-31 G$ *

Reference energy:

Total energy:
$-302.9213175733$

$-303.8127531762$

$\operatorname{CASSCF}(4,4) / 6-31 G$ *

$E=-302.8769602364$

Sum of electronic and zero-point Energies=

Sum of electronic and thermal Energies=

Sum of electronic and thermal Enthalpies=

Sum of electronic and thermal Free Energies=

$$
\begin{aligned}
& -302.737773 \\
& -302.733076 \\
& -302.732131 \\
& -302.766051
\end{aligned}
$$

$$
\text { E (Thermal) }
$$$$
\mathrm{KCAL} / \mathrm{MOL}
$$

TOTAL

$$
90.289
$$

CV

CAL/MOL - KELVIN

18.579
1.098796

.778720

.000000

$-.778720$

1.191382

1.208962

$-1.208962$

$-1.098796$

$-1.191382$

$-.625467$

$-2.139314$

.000000

.625467

.000000

2.139314 


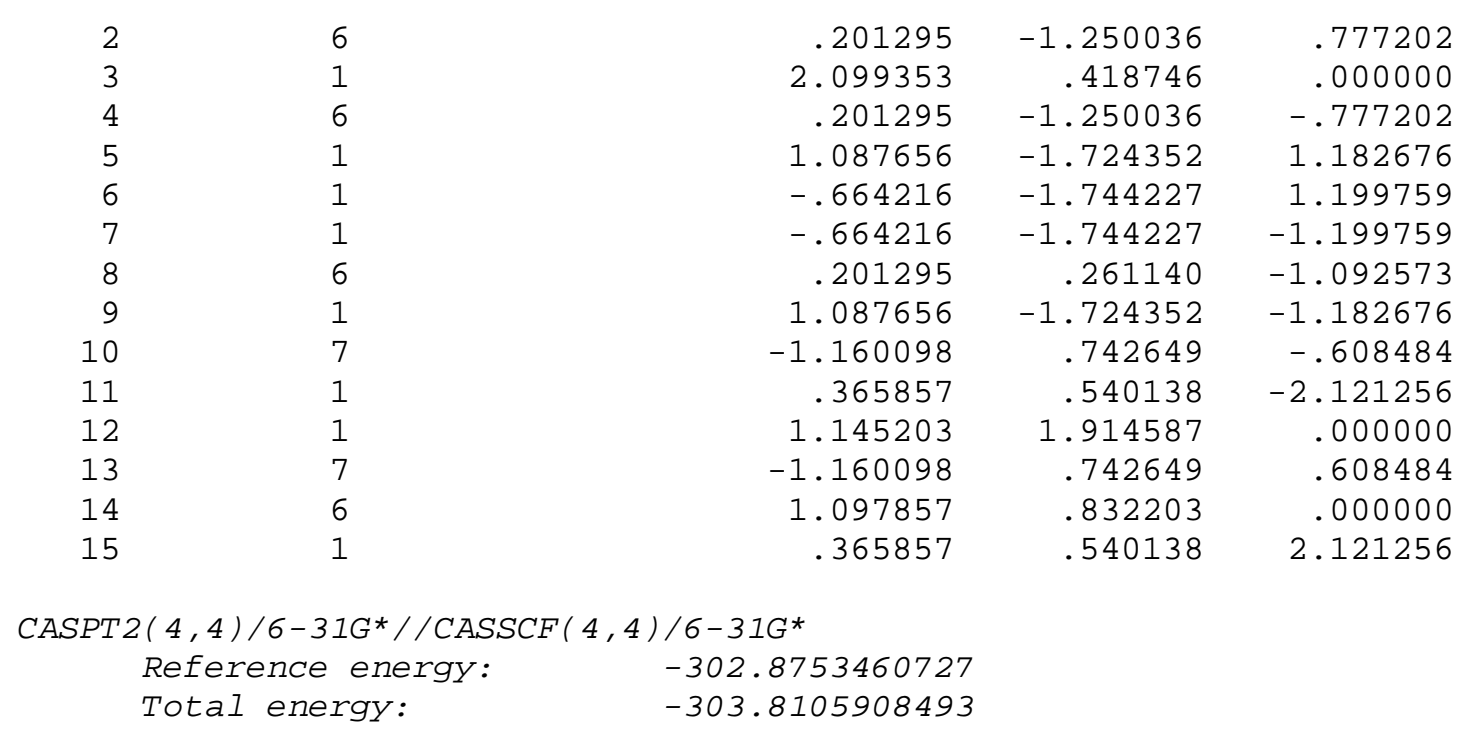

$\operatorname{CASSCF}(10,8) / 6-31 G^{*}$

$E=-302.9320724427$

Sum of electronic and zero-point Energies= $\quad-302.793744$

Sum of electronic and thermal Energies= -302.788981

Sum of electronic and thermal Enthalpies= $\quad-302.788037$

Sum of electronic and thermal Free Energies= $\quad-302.822067$

$\begin{array}{ll}\text { TOTAL } & 89.791\end{array}$

E (Thermal) $\mathrm{KCAL} / \mathrm{MOL}$
CV

CAL/MOL-KELVIN CAL/MOL-KELVIN 18.866
71.622

$\begin{array}{rr}1 & 6 \\ 2 & 6 \\ 3 & 1 \\ 4 & 6 \\ 5 & 1 \\ 6 & 1 \\ 7 & 1 \\ 8 & 6 \\ 9 & 1 \\ 10 & 7 \\ 11 & 1 \\ 12 & 1 \\ 13 & 7 \\ 14 & 6 \\ 15 & 1\end{array}$

.208343
.208343
2.098668
.208343
1.093870
-.657118
-.657118
.208343
1.093870
-1.172388
.376224
1.131480
-1.172388
1.092852
.376224

.258504

$-1.248779$

.435460

$-1.248779$

$-1.726099$

$-1.744577$

$-1.744577$

.258504

$-1.726099$

.739876

.538630

1.921652

.739876

.838670

.538630
1.095878

.778007

.000000

$-.778007$

1.181810

1.199727

$-1.199727$

$-1.095878$

$-1.181810$

$-.618675$

$-2.123762$

.000000

.618675

.000000

2. 123762

CASPT2 $(10,8) / 6-31 G^{*} / / \operatorname{CASSCF}(10,8) / 6-31 G^{*}$

Reference energy:

$-302.9304039562$

Total energy:

$-303.8135162736$

DBH: Transition structure: 2-bond cleavage TS(B)

UB3LYP/6-31G(d)

$E(U B+H F-L Y P)=-304.728970158$ 
Sum of electronic and zero-point Energies=

Sum of electronic and thermal Energies=

Sum of electronic and thermal Enthalpies=

Sum of electronic and thermal Free Energies=
-304.607834
-304.601168
-304.600224
-304.638256

CV

CAL/MOL-KELVIN CAL/MOL-KELVIN 24.745
80.045

TOTAL

E (Thermal) $\mathrm{KCAL} / \mathrm{MOL}$ 80.197
$-1.078365$

$-0.149543$

$-0.214125$

$-1.102416$

$-1.100523$

$-0.210732$

0.691080

$-0.103218$

1.640208

$-0.736879$

$-2.122606$

$-2.119669$

$-0.734457$

$-0.097655$

1. 640621
$-0.001325$
- 0.002109
$-1.167992$
$-0.783307$
0.787384
1.166489
$-0.004224$
$-2.196795$
$-0.572463$
$-1.206099$
$-1.173301$
1.179450
1. 212142
2. 194119
$\odot .571498$

1.996304

1. 391239

0.459079

$-0.706686$

$-0.704372$

0.462166

2. 091598

$\odot .785515$

$-0.474931$

$-1.650109$

$-0.572895$

$-0.568787$

$-1.646704$

0.791640

$-0.472943$

CASPT2 $(6,6) / 6-31 G * / / B 3 L Y P / 6-31 G$ *

Reference energy:

Total energy:
$-302.8770260716$

$-303.7481402172$

$\operatorname{CASSCF}(4,4) / 6-31 G$ *

$E=-302.8201244630$

Sum of electronic and zero-point Energies=

Sum of electronic and thermal Energies=

$-302.689600$

$-302.683493$

$-302.682549$

$-302.719523$

Sum of electronic and thermal Free Energies=

E (Thermal) $\mathrm{KCAL} / \mathrm{MOL}$

TOTAL 85.737
CV CAL/MOL-KELVIN CAL/MOL-KELVIN 22.279
77.817

$\begin{array}{rr}1 & 1 \\ 2 & 6 \\ 3 & 6 \\ 4 & 6 \\ 5 & 6 \\ 6 & 6 \\ 7 & 1 \\ 8 & 1 \\ 9 & 7 \\ 10 & 1 \\ 11 & 1 \\ 12 & 1 \\ 13 & 1 \\ 14 & 1 \\ 15 & 7\end{array}$

-1.066227
-0.133933
-0.156602
-1.116322
-1.115187
-0.154707
0.691367
-0.080373
1.601620
-0.805201
-2.112669
-2.110911
-0.803686
-0.077099
1.601995

$-0.000782$

$-0.001194$

$-1.156782$

$-0.779789$

0.782133

1.155899

$-0.002394$

$-2.176304$

$-0.566588$

$-1.197019$

$-1.165899$

1.169366

1.200517

2.174780

$\odot .566035$
1.961886

1.393068

0.438431

$-0.679146$

$-0.677852$

0.440179

2. 093601

0.773044

$-0.491904$

$-1.630201$

$-0.479092$

$-0.476889$

$-1.628273$

0.776442

$-\odot .490753$ 


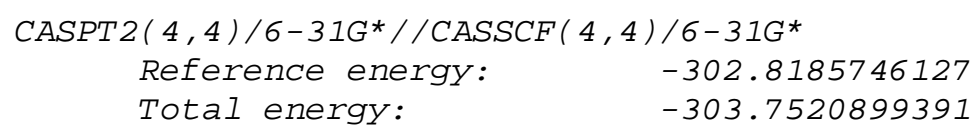

E (Thermal) $\mathrm{KCAL} / \mathrm{MOL}$

TOTAL 85.679
CV CAL/MOL-KELVIN CAL/MOL-KELVIN 22.157
77.688

$\begin{array}{rr}1 & 1 \\ 2 & 6 \\ 3 & 6 \\ 4 & 6 \\ 5 & 6 \\ 6 & 6 \\ 7 & 1 \\ 8 & 1 \\ 9 & 7 \\ 10 & 1 \\ 11 & 1 \\ 12 & 1 \\ 13 & 1 \\ 14 & 1 \\ 15 & 7\end{array}$

$$
\begin{array}{r}
-1.043936 \\
-.111035 \\
-.138169 \\
-1.120254 \\
-1.118679 \\
-.135843 \\
.714239 \\
-.067306 \\
1.576994 \\
-.828415 \\
-2.112939 \\
-2.110529 \\
-.825780 \\
-.064053 \\
1.577664
\end{array}
$$

1.957106

1.389953

.435662

$-.664172$

$-.663022$

.437540

2.090720

.774245

$-.506860$

$-1.621434$

$-.445435$

$-.443588$

$-1.619592$

.778220

$-.505426$

$\operatorname{CASPT2}(10,8) / 6-31 G * / / \operatorname{CASSCF}(10,8) / 6-31 G *$

Reference energy: $\quad-302.8864885324$

Total energy: $\quad-303.7548974034$

\section{Intermediate: singlet cyclopentane-1,4-diyl}

UB3LYP/6-31G(d)

$E(U B+H F-L Y P)=-195.228946852$

Sum of electronic and zero-point Energies= $\quad-195.117691$

Sum of electronic and thermal Energies=

Sum of electronic and thermal Enthalpies=

$-195.111139$

Sum of electronic and thermal Free Energies=

$-195.146014$

E (Thermal) $\mathrm{KCAL} / \mathrm{MOL}$

TOTAL

$\begin{array}{ll}1 & 6 \\ 2 & 6 \\ 3 & 1 \\ 4 & 6 \\ 5 & 1 \\ 6 & 1 \\ 7 & 1\end{array}$

73.290

$$
6
$$

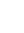$$
1
$$$$
1
$$

CV CAL/MOL-KELVIN 19.833
$S$

$\mathrm{CAL} / \mathrm{MOL}-\mathrm{KELVIN}$

73.400

.408690
-1.024859
2.022538
-1.025074
-1.400090
-1.696461
-1.400808
1.174730
$-.078208$
.772206 .090287 .053037 .873569
$-.771970 \quad-.090224$
$1.039144 \quad 1.094794$
$1.278137 \quad-.617982$
$-1.038773 \quad-1.094574$ 


$\begin{array}{rrrrr}8 & 6 & .408419 & -1.174817 & .077758 \\ 9 & 1 & -1.696511 & -1.277824 & .618267 \\ 10 & 1 & .742175 & -2.206755 & .119361 \\ 11 & 1 & 2.023458 & -.053436 & -.872742 \\ 12 & 6 & 1.343309 & -.000174 & .000001 \\ 13 & 1 & .742791 & 2.206627 & -.118373\end{array}$
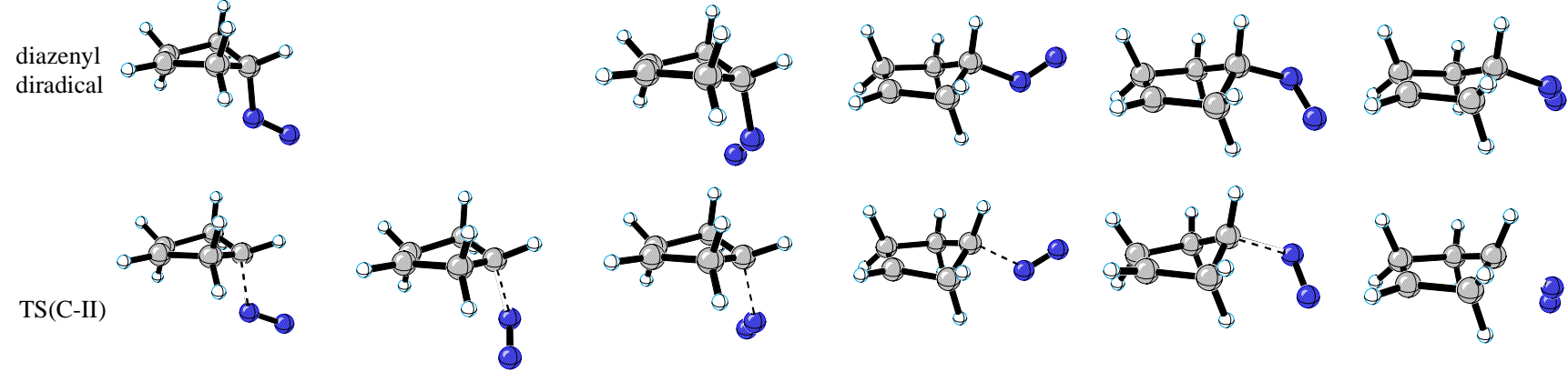

$\mathrm{a}-\mathrm{X}$

a-n1

a-n2

b-x

b-n1

b-n2

DBH intermediate: diazenyl dirad, envelope a, exo $\mathrm{N}=\mathrm{N}$

UB3LYP/6-31G(d)

$E(U B+H F-L Y P)=-304.733820359$

Sum of electronic and zero-point Energies= $\quad-304.610804$

Sum of electronic and thermal Energies=

$-304.603644$

Sum of electronic and thermal Enthalpies=

$-304.602700$

Sum of electronic and thermal Free Energies=

$-304.642103$

TOTAL

E (Thermal) $\mathrm{KCAL} / \mathrm{MOL}$

CV

$S$

\begin{tabular}{rrr}
\multicolumn{2}{c}{ CV } & \multicolumn{1}{c}{$\mathrm{S}$} \\
\multicolumn{2}{c}{ CAL/MOL-KELVIN } & CAL/MOL-KELVIN \\
25.703 & & 82.931 \\
-.451304 & .073188 & .651971 \\
.496177 & -1.136853 & .588444 \\
.810724 & 1.689583 & 1.346415 \\
1.544399 & -.763628 & -.487050 \\
.977492 & -1.240772 & 1.568641 \\
-.035604 & -2.070207 & .380243 \\
1.232622 & -1.136927 & -1.476752 \\
1.567148 & .735183 & -.453219 \\
2.524570 & -1.219506 & -.286920 \\
-2.604666 & -.073110 & -.350584 \\
2.266097 & 1.347550 & -1.012126 \\
-.094869 & 2.112939 & -.101044 \\
-1.438830 & -.043852 & -.517246 \\
.453148 & 1.291557 & .379970 \\
-1.033968 & .139390 & 1.575658
\end{tabular}

CASPT2 $(6,6) / 6-31 G^{*} / / B 3 L Y P / 6-31 G^{*}$

Reference energy:

Total energy:
$-302.8649785397$

$-303.7468227263$ 


\begin{tabular}{|c|c|c|c|c|c|c|}
\hline \multicolumn{7}{|c|}{$\operatorname{CASSCF}(4,4) / 6-31 G^{*}$} \\
\hline $\mathrm{E}=$ & -302.8211321 & 367 & & & & \\
\hline Sum 0 & of electronic & and zero-point & nergies= & & -302 & 689101 \\
\hline Sum 0 & of electronic & and thermal En & gies= & & -302 & 682322 \\
\hline Sum 0 & of electronic & and thermal En & alpies= & & -302 & 681378 \\
\hline Sum 0 & of electronic & and thermal $\mathrm{Fr}$ & Energies= & & -302 & 720006 \\
\hline & & E (Thermal) & & & & $\mathrm{S}$ \\
\hline & & KCAL/MOL & $\mathrm{CAL} / \mathrm{MOL}$ & ELVIN & & /MOL - KELVIN \\
\hline TOTAL & & 87.104 & & 944 & & 81.300 \\
\hline 1 & 6 & & -0.454346 & 0.07 & 6920 & 0.648563 \\
\hline 2 & 6 & & 0.489701 & -1.12 & 7122 & 0.599825 \\
\hline 3 & 1 & & 0.794482 & 1.68 & 9493 & 1.338024 \\
\hline 4 & 6 & & 1.516721 & -0.77 & 1188 & $-\odot .49 \odot 201$ \\
\hline 5 & 1 & & $\odot .982152$ & -1.21 & 1356 & 1.564480 \\
\hline 6 & 1 & & -0.034911 & -2.05 & 6933 & 0.415529 \\
\hline 7 & 1 & & 1.169236 & -1.10 & 2177 & -1.466405 \\
\hline 8 & 6 & & 1.588201 & 0.73 & 2232 & -0.423712 \\
\hline 9 & 1 & & 2.478096 & -1.24 & 6597 & -0.318672 \\
\hline 10 & 7 & & -2.563659 & -0.07 & 2250 & -0.331863 \\
\hline 11 & 1 & & 2.133725 & 1.32 & 0787 & -1.136764 \\
\hline 12 & 1 & & -0.087419 & 2.09 & 2208 & -0.114152 \\
\hline 13 & 7 & & -1.424223 & -0.04 & 7852 & -0.548384 \\
\hline 14 & 6 & & 0.446206 & 1.29 & 1007 & 0.386072 \\
\hline 15 & 1 & & -1.039083 & 0.14 & 4191 & 1.556407 \\
\hline
\end{tabular}

CASPT2 $(4,4) / 6-31 G * / / \operatorname{CASSCF}(4,4) / 6-31 G^{*}$

Reference energy: $\quad-302.8194628662$

Total energy: $\quad-303.7488075048$

$\begin{array}{ll}\operatorname{CASSCF}(10,8) / 6-31 \mathrm{G}^{*} & \\ \mathrm{E}=-302.8844722262 & -302.753796 \\ \text { Sum of electronic and zero-point Energies= } & -302.746657 \\ \text { Sum of electronic and thermal Energies= } & -302.745713 \\ \text { Sum of electronic and thermal Enthalpies= } & -302.785258\end{array}$

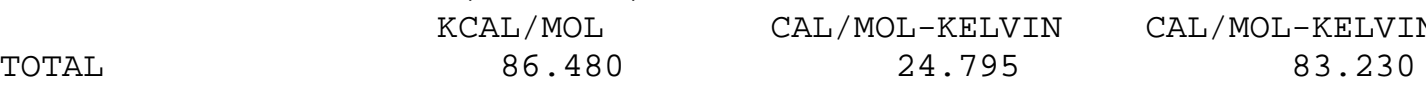

E (Thermal) CV $\quad$ C

$\begin{array}{rrrrr}1 & 6 & -0.421550 & 0.088974 & 0.680700 \\ 2 & 6 & 0.501993 & -1.125155 & 0.610692 \\ 3 & 1 & 0.849874 & 1.701373 & 1.322224 \\ 4 & 6 & 1.507419 & -0.781909 & -0.504332 \\ 5 & 1 & 1.017562 & -1.217926 & 1.562795 \\ 6 & 1 & -0.037431 & -2.049122 & 0.439089 \\ 7 & 1 & 1.137590 & -1.117587 & -1.470858 \\ 8 & 6 & 1.590374 & 0.721822 & -0.450043 \\ 9 & 1 & 2.469402 & -1.262194 & -0.350975 \\ 10 & 7 & -2.600815 & -0.075185 & -0.332359 \\ 11 & 1 & 2.125395 & 1.301879 & -1.177815 \\ 12 & 1 & -0.068723 & 2.094298 & -0.109160\end{array}$




$\begin{array}{rrrrr}13 & 7 & -1.455187 & -0.039496 & -0.543258 \\ 14 & 6 & 0.473113 & 1.294321 & 0.384574 \\ 15 & 1 & -1.009749 & 0.163721 & 1.584470\end{array}$
CASPT2 $(10,8) / 6-31 G * / / \operatorname{CASSCF}(10,8) / 6-31 G *$
Reference energy: $\quad-302.8827346088$
Total energy: $\quad-303.7472303113$

\section{$\underline{\text { DBH intermediate: diazenyl dirad, envelope } \mathrm{a} \text {, endo2 } \mathrm{N}=\mathrm{N}}$}

UB3LYP/6-31G(d)

$E(U B+H F-$ LYP $)=-304.733429677$

Sum of electronic and zero-point Energies= $\quad-304.610403$

Sum of electronic and thermal Energies= $\quad-304.603285$

Sum of electronic and thermal Enthalpies= $\quad-304.602340$

Sum of electronic and thermal Free Energies= $\quad-304.641595$

$\begin{array}{cccc} & \text { E (Thermal) } & \text { CV } & \text { S } \\ \text { KCAL/MOL } & \text { CAL/MOL-KELVIN } & \text { CAL/MOL-KELVIN } \\ \text { TOTAL } & 81.667 & 25.667 & 82.619\end{array}$

$\begin{array}{rrrrr}1 & 6 & -0.378794 & 0.455957 & 0.741207 \\ 2 & 6 & 0.246537 & -0.936425 & 0.888247 \\ 3 & 1 & 1.278897 & 1.874444 & 0.847004 \\ 4 & 6 & 1.168800 & -1.076240 & -0.345099 \\ 5 & 1 & 0.840314 & -0.953646 & 1.810151 \\ 6 & 1 & -0.510870 & -1.722440 & 0.956655 \\ 7 & 1 & 0.618723 & -1.548060 & -1.177911 \\ 8 & 6 & 1.547739 & 0.336940 & -0.666980 \\ 9 & 1 & 2.034569 & -1.724126 & -0.149512 \\ 10 & 7 & -2.075184 & -0.494040 & -0.687829 \\ 11 & 1 & 2.322421 & 0.615601 & -1.373145 \\ 12 & 1 & 0.253800 & 2.102951 & -0.564042 \\ 13 & 7 & -1.611512 & 0.466756 & -0.195223 \\ 14 & 6 & 0.705120 & 1.330829 & 0.076214 \\ 15 & 1 & -0.767401 & 0.879904 & 1.670635\end{array}$

DBH intermediate: diazenyl dirad, envelope $b$, exo $\mathrm{N}=\mathrm{N}$

UB3LYP/6-31G(d)

$\mathrm{E}(\mathrm{UB}+\mathrm{HF}-\mathrm{LYP})=-304.734297212$

Sum of electronic and zero-point Energies= $\quad-304.611107$

Sum of electronic and thermal Energies= $\quad-304.603967$

Sum of electronic and thermal Enthalpies= $\quad-304.603022$

Sum of electronic and thermal Free Energies= $\quad-304.642367$

$\begin{array}{cccc} & \text { KCAL/MOL } & \text { CAL/MOL-KELVIN } & \text { CAL/MOL - KELVIN } \\ \text { TOTAL } & 81.784 & 25.656 & 82.807\end{array}$

$\begin{array}{rrrrr}1 & 7 & -2.794742 & -0.079226 & 0.209241 \\ 2 & 7 & -1.778297 & 0.013760 & -0.379557 \\ 3 & 6 & -0.423270 & 0.015161 & 0.312224 \\ 4 & 6 & 0.424483 & -1.173471 & -0.170996 \\ 5 & 6 & 1.881639 & -0.716235 & 0.083857 \\ 6 & 6 & 1.801939 & 0.780128 & 0.008583\end{array}$




$\begin{array}{rrrrr}7 & 6 & 0.387932 & 1.259641 & -0.097510 \\ 8 & 1 & 0.112403 & 1.537939 & -1.131860 \\ 9 & 1 & 0.164032 & 2.134497 & 0.526492 \\ 10 & 1 & 0.251614 & -1.311046 & -1.245174 \\ 11 & 1 & 0.161722 & -2.108235 & 0.332767 \\ 12 & 1 & 2.586402 & -1.152966 & -0.638842 \\ 13 & 1 & 2.227671 & -1.052486 & 1.076551 \\ 14 & 1 & 2.661740 & 1.439275 & 0.061055 \\ 15 & 1 & -0.590646 & -0.020060 & 1.394279\end{array}$

CASPT2 $(6,6) / 6-31 G * / / U B 3 L Y P / 6-31 G$ *

Reference energy: $\quad-302.8645305618$

Total energy: $\quad-303.7463374396$

$\operatorname{CASSCF}(4,4) / 6-31 G^{*}$
$\mathrm{E}=\quad-302.8210108402$

Sum of electronic and zero-point Energies=

Sum of electronic and thermal Energies=

Sum of electronic and thermal Enthalpies=

Sum of electronic and thermal Free Energies=

E (Thermal) $\mathrm{KCAL} / \mathrm{MOL}$

TOTAL

87.121
-2.744494
-1.793812
-0.421707
0.419171
1.862087
1.805844
0.381443
-0.571971
0.141359
0.278185
2.143855
2.585542
2.620249
0.171852
0.118046

CV

-KELVIN

$-302.689023$

$-302.682175$

$-302.681231$

$-302.720048$

$12 \quad 1$

$13 \quad 1$

$15 \quad 1$

CASPT2 $(4,4) / 6-31 G * / / \operatorname{CASSCF}(4,4) / 6-31 G$ *

Reference energy:

$-302.8193676446$

Total energy:

$-303.7481905219$

$S$

CAL/MOL-KELVIN

81.698

\section{DBH intermediate: diazenyl dirad, envelope $b$, endo1 $\mathrm{N}=\mathrm{N}$}

UB3LYP/6-31G(d)

$E(U B+H F-$ LYP $)=-304.733961573$

Sum of electronic and zero-point Energies= $\quad-304.610776$

Sum of electronic and thermal Energies= $\quad-304.603667$

Sum of electronic and thermal Enthalpies= $\quad-304.602723$

Sum of electronic and thermal Free Energies= $\quad-304.641890$
E (Thermal)
CV
S 
TOTAL

$\begin{array}{rr}1 & 7 \\ 2 & 7 \\ 3 & 6 \\ 4 & 6 \\ 5 & 6 \\ 6 & 6 \\ 7 & 6 \\ 8 & 1 \\ 9 & 1 \\ 10 & 1 \\ 11 & 1 \\ 12 & 1 \\ 13 & 1 \\ 14 & 1 \\ 15 & 1\end{array}$

$\mathrm{KCAL} / \mathrm{MOL}$

81.761
CAL/MOL-KELVIN 25.628
CAL/MOL - KELVIN

82.434

-2.479566
-1.845106
-0.373112
0.515703
1.913405
1.614419
0.156008
2.505895
2.502913
2.382140
-0.077130
-0.351905
-0.297566
0.496888
0.152934

0.237347
-0.424433
-0.147690
-1.200343
-0.534861
0.936865
1.207501
-0.852666
-0.830783
1.700167
2.042033
1.433466
-0.231969
-2.169947
-1.349531

$-0.490979$

0.246730

$\odot .557989$

$-0.133928$

$-0.151998$

$-0.095505$

0.051258

0.722348

$-1.032845$

$-0.023226$

0.724019

$-0.906955$

1. 646186

0.371107

$-1.157788$

$\underline{\text { DBH intermediate: diazenyl dirad, envelope } b \text {, endo2 } \mathrm{N}=\mathrm{N}}$

UB3LYP/6 - 31G(d)

$\mathrm{E}(\mathrm{UB}+\mathrm{HF}-\mathrm{LYP})=-304.732234198$

Sum of electronic and zero-point Energies=

Sum of electronic and thermal Energies=

Sum of electronic and thermal Enthalpies=

Sum of electronic and thermal Free Energies=

$-304.609199$

$-304.601973$

$-304.601029$

$-304.640647$

E (Thermal) $\mathrm{KCAL} / \mathrm{MOL}$ 81.740
CV

CAL/MOL-KELVIN CAL/MOL-KELVIN 25.729
$S$ 83.384

TOTAL

6
6
6
1
1
6
1
1
6
1
1
7
7
1
1

0.502709
-0.387927
0.215045
-0.370298
-0.145801
1.737569
-0.074599
2.260483
1.835717
0.510480
2.194709
-1.850362
-2.530042
0.104897
2.764293

1. 291711

$-0.095128$

0.180950

$\odot .504500$

$-1.140026$

0.002933

0.239831

1.598139

$-1.328843$

$-1.014687$

$-0.866643$

$-0.005250$

$-1.996607$

$-1.443845$

$\odot .618258$

$-0.781459$

0.618110

$-0.203696$

2.202452

0.518006

$-1.175266$

0.951462

0.420795

0.146947

$-0.383931$

$-0.381197$

1.592189

$-1.081293$

1.147426

$-0.388826$

DBH transition structure: cleave 2nd CN TS(C-II), envelope a, exo $N=N$

UB3LYP/6-31G(d)

$E(U B+H F-L Y P)=-304.729748615$

Sum of electronic and zero-point Energies=

Sum of electronic and thermal Energies=

$-304.609391$

Sum of electronic and thermal Enthalpies=

$-304.602020$

Sum of electronic and thermal Free Energies=

$-304.601076$

$-304.641796$ 
E (Thermal) $\mathrm{KCAL} / \mathrm{MOL}$ 80.151
CV CAL/MOL-KELVIN CAL/MOL-KELVIN 25.378
$S$ 85.701

$\begin{array}{rr}1 & 6 \\ 2 & 6 \\ 3 & 1 \\ 4 & 6 \\ 5 & 1 \\ 6 & 1 \\ 7 & 1 \\ 8 & 6 \\ 9 & 1 \\ 10 & 7 \\ 11 & 1 \\ 12 & 1 \\ 13 & 7 \\ 14 & 6 \\ 15 & 1\end{array}$

$$
\begin{array}{r}
.138607 \\
-1.128199 \\
1.802998 \\
-.823712 \\
-1.294180 \\
-2.017596 \\
-1.179501 \\
.670638 \\
-1.339972 \\
-.090895 \\
1.231096 \\
2.087796 \\
.014361 \\
1.308207 \\
.251849
\end{array}
$$

$-.962622$
.751390
.625534
1.244451
$-.511489$
1.572182 .443444
$-1.477620$
$-.492095$
$-.370537$
$-.291714$
$-1.086413$
$-.188040$
$-.576094$
.343820
1.634225

CASPT2 $(6,6) / 6-31 G^{*} / / B 3 L Y P / 6-31 G *$

$\begin{array}{ll}\text { Reference energy: } & -302.8701353653 \\ \text { Total energy: } & -303.7397371686\end{array}$

$\begin{array}{ll}\operatorname{CASSCF}(4,4) / 6-31 \mathrm{G}^{*} & \\ \mathrm{E}=-302.8180337556 & -302.688536 \\ \text { Sum of electronic and zero-point Energies= } & -302.681672 \\ \text { Sum of electronic and thermal Energies= } & -302.680728 \\ \text { Sum of electronic and thermal Enthalpies= } & -302.719925 \\ \text { Sum of electronic and thermal Free Energies= }\end{array}$

$\begin{array}{cccc} & & \mathrm{CV} & \mathrm{S} \\ \mathrm{KCAL}(\text { Thermal) } & \mathrm{CAL} / \mathrm{MOL}-\mathrm{KELVIN} & \mathrm{CAL} / \mathrm{MOL}-\mathrm{KELVIN} \\ \text { TOTAL } & 85.568 & 23.503 & 82.498\end{array}$

$\begin{array}{rrrrr}1 & 6 & -0.375294 & 0.107667 & 0.725206 \\ 2 & 6 & 0.508401 & -1.125786 & 0.621820 \\ 3 & 1 & 0.878955 & 1.768880 & 1.273866 \\ 4 & 6 & 1.493276 & -0.797123 & -0.515509 \\ 5 & 1 & 1.047016 & -1.245897 & 1.559147 \\ 6 & 1 & -0.059474 & -2.033432 & 0.454242 \\ 7 & 1 & 1.085792 & -1.111036 & -1.474447 \\ 8 & 6 & 1.618916 & 0.703037 & -0.450357 \\ 9 & 1 & 2.445837 & -1.304558 & -0.395368 \\ 10 & 7 & -2.619127 & -0.067529 & -0.288525 \\ 11 & 1 & 2.165489 & 1.270010 & -1.180455 \\ 12 & 1 & -0.055805 & 2.067375 & -0.168907 \\ 13 & 7 & -1.524387 & -0.039946 & -0.591672 \\ 14 & 6 & 0.500165 & 1.302716 & 0.364778 \\ 15 & 1 & -0.975999 & 0.197923 & 1.617676\end{array}$

CASPT2 $(4,4) / 6-31 G * / / \operatorname{CASSCF}(4,4) / 6-31 G^{*}$

Reference energy: $\quad-302.8163768516$

Total energy: $\quad-303.7426807562$ 


\begin{tabular}{|c|c|c|c|c|c|c|}
\hline \multicolumn{7}{|c|}{$\operatorname{CASSCF}(10,8) / 6-31 G^{*}$} \\
\hline$E=$ & -302.8843904 & 151 & & & & \\
\hline Sum 0 & of electronic & and zero-point & nerqies= & & -302 & 754831 \\
\hline Sum 0 & of electronic & and thermal Ene & gies= & & -302 & 747998 \\
\hline Sum 0 & of electronic & and thermal Ent & alpies= & & -302 & 747054 \\
\hline Sum 0 & of electronic & and thermal Fre & Energies= & & -302 & 786245 \\
\hline & & E (Thermal) & & & & S \\
\hline & & KCAL/MOL & $\mathrm{CAL} / \mathrm{MOL}$ & ELVIN & & /MOL-KELVIN \\
\hline TOTAL & & 85.587 & & 378 & & 82.483 \\
\hline 1 & 6 & & -0.398554 & 0.10 & 1941 & 0.701193 \\
\hline 2 & 6 & & 0.503725 & -1.12 & 3825 & 0.618465 \\
\hline 3 & 1 & & 0.876623 & 1.72 & 8543 & 1.299275 \\
\hline 4 & 6 & & 1.498377 & -0.79 & 3684 & -0.510137 \\
\hline 5 & 1 & & 1.031991 & -1.22 & 6833 & 1.563000 \\
\hline 6 & 1 & & -0.050940 & -2.04 & 0037 & 0.453274 \\
\hline 7 & 1 & & 1.108925 & -1.12 & 2148 & -1.471604 \\
\hline 8 & 6 & & 1.604620 & 0.70 & 8568 & -0.455824 \\
\hline 9 & 1 & & 2.455345 & -1.28 & 8308 & -0.372164 \\
\hline 10 & 7 & & -2.618464 & -0.07 & 5147 & -0.317960 \\
\hline 11 & 1 & & 2.145421 & 1.27 & 9579 & -1.186683 \\
\hline 12 & 1 & & -0.052195 & 2.08 & 7418 & -0.133915 \\
\hline 13 & 7 & & -1.485812 & -0.03 & 2723 & -0.556567 \\
\hline 14 & 6 & & 0.492829 & 1.29 & 8609 & 0.374652 \\
\hline 15 & 1 & & -0.991221 & 0.18 & 7211 & 1.600410 \\
\hline
\end{tabular}

$\operatorname{CASPT2}(10,8) / 6-31 G * / / \operatorname{CASSCF}(10,8) / 6-31 G$ *

Reference energy: $\quad-302.8826594158$

Total energy: $\quad-303.7452446275$

\section{DBH transition structure: cleave 2nd CN TS(C-II), envelope $a$, endo1 $\mathrm{N}=\mathrm{N}$}

UB3LYP/6-31G(d)

$E(U B+H F-L Y P)=-304.730978798$

Sum of electronic and zero-point Energies= $\quad-304.610183$

Sum of electronic and thermal Energies= $\quad-304.603012$

Sum of electronic and thermal Enthalpies= $\quad-304.602068$

Sum of electronic and thermal Free Energies= $\quad-304.641727$

TOTAL

E (Thermal) $\mathrm{KCAL} / \mathrm{MOL}$ 80.300
CV CAL/MOL-KELVIN CAL/MOL-KELVIN 25.093

83.469

$\begin{array}{rrr}-0.285739 & -\odot .244645 & \odot .853962 \\ 0.793471 & -1.200725 & 0.382977 \\ 0.544808 & 1.637455 & 1.567178 \\ 1.532692 & -0.425478 & -0.734747 \\ 1.479470 & -1.407572 & 1.216171 \\ 0.378221 & -2.159511 & 0.054679 \\ 1.095208 & -\odot .669227 & -1.719437 \\ 1.309449 & 1.011961 & -0.375603 \\ 2.595320 & -\odot .695191 & -\odot .80315 \odot\end{array}$




$\begin{array}{rrrrr}10 & 7 & -2.296169 & 0.304186 & -\odot .649360 \\ 11 & 1 & 1.834769 & 1.842577 & -0.834673 \\ 12 & 1 & -0.620562 & 1.823804 & 0.263884 \\ 13 & 7 & -1.682372 & -0.565974 & -0.214601 \\ 14 & 6 & 0.200770 & 1.180329 & 0.622506 \\ 15 & 1 & -0.761306 & -0.468478 & 1.808503\end{array}$

DBH transition structure: cleave 2nd CN TS(C-II), envelope a, endo2 $\mathrm{N}=\mathrm{N}$

UB3LYP/6-31G(d)

$E(U B+H F-L Y P)=-304.730911212$

Sum of electronic and zero-point Energies= $\quad-304.610095$

Sum of electronic and thermal Energies= $\quad-304.602914$

Sum of electronic and thermal Enthalpies= -304.601970

Sum of electronic and thermal Free Energies= $\quad-304.641703$

$\mathrm{KCAL} / \mathrm{MOL}$
TOTAL
80.319

CV $S$

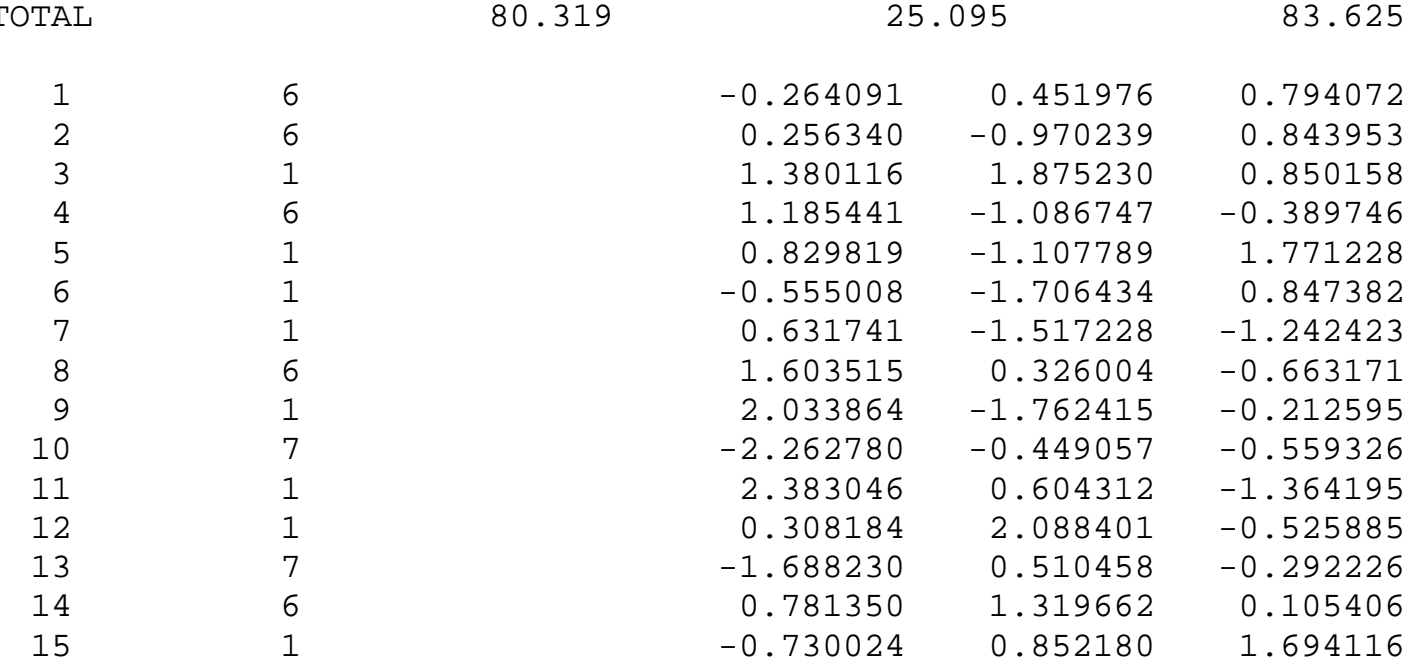

DBH transition structure: cleave 2nd CN TS(C-II), envelope b, exo N=N

UB3LYP/6-31G(d)

$E(U B+H F-L Y P)=-304.728677372$

Sum of electronic and zero-point Energies= $\quad-304.608174$

Sum of electronic and thermal Energies= $\quad-304.600745$

Sum of electronic and thermal Enthalpies= -304.599801

Sum of electronic and thermal Free Energies= $\quad-304.640349$

80.279
CV

CAL/MOL-KELVIN CAL/MOL-KELVIN 25.535
$S$ 85.342

$\begin{array}{ll}1 & 7 \\ 2 & 7 \\ 3 & 6 \\ 4 & 6 \\ 5 & 6 \\ 6 & 6 \\ 7 & 6 \\ 8 & 1\end{array}$

-2.907014
-1.944588
-0.313436
0.447278
1.934133
1.852007
0.436352
2.406834

$-0.096161$

$\odot .035571$

0.035777

$-1.178908$

$-0.726834$

0.773387

1.263181

$-1.097669$
0.176281

$-0.428130$

0.421604

$-0.082371$

$-0.018423$

$-0.023785$

$-0.077964$

๑. 905075 


$\begin{array}{rrrrr}9 & 1 & 2.537299 & -1.133917 & -0.845495 \\ 10 & 1 & 0.244846 & -2.084084 & 0.496719 \\ 11 & 1 & 0.158039 & -1.378407 & -1.122680 \\ 12 & 1 & -0.592864 & 0.033958 & 1.474906 \\ 13 & 1 & 0.255866 & 2.161613 & 0.524484 \\ 14 & 1 & 0.102755 & 1.501821 & -1.107024 \\ 15 & 1 & 2.710433 & 1.421188 & 0.122585\end{array}$

CASPT2 $(6,6) / 6-31 G * / / U B 3 L Y P / 6-31 G *$

Reference energy: $\quad-302.8714382251$

Total energy: $\quad-303.7395119751$

$\begin{array}{ll}\operatorname{CASSCF}(4,4) / 6-31 \mathrm{G}^{*} & \\ \mathrm{E}=-302.8177361371 & -302.688046 \\ \text { Sum of electronic and zero-point Energies= } & -302.681139 \\ \text { Sum of electronic and thermal Energies= } & -302.680195 \\ \text { Sum of electronic and thermal Enthalpies= } & -302.719513\end{array}$

Sum of electronic and thermal Free Energies=

E (Thermal)
KCAL/MOL
TOTAL
85.716

CV

$\mathrm{S}$

$\begin{array}{rr}1 & 7 \\ 2 & 7 \\ 3 & 6 \\ 4 & 6 \\ 5 & 6 \\ 6 & 6 \\ 7 & 6 \\ 8 & 1 \\ 9 & 1 \\ 10 & 1 \\ 11 & 1 \\ 12 & 1 \\ 13 & 1 \\ 14 & 1 \\ 15 & 1\end{array}$

$\begin{array}{lr}7 & 2.803707 \\ 7 & 1.911531 \\ 6 & 0.351744 \\ 6 & -0.422113 \\ 6 & -1.893932 \\ 6 & -1.839837 \\ 6 & -0.425253 \\ 1 & -2.265658 \\ 1 & -2.552892 \\ 1 & -0.165763 \\ 1 & -0.200910 \\ 1 & 0.531664 \\ 1 & -0.209813 \\ 1 & -0.177993 \\ 1 & -2.588959\end{array}$

CAL/MOL-KELVIN

CAL/MOL-KELVIN 23.582

82.750

CASPT2 $(4,4) / 6-31 G * / / \operatorname{CASSCF}(4,4) / 6-31 G^{*}$
$-0.108116$
$-0.270484$
0.042956
0.416313
0.051207
$-0.341745$
$-1.163129$
0.155540
$-0.740821$
$-0.038062$
0.772772
$-0.014846$
1.258536
$\odot .158485$
$-1.098793$
$-0.995135$
$-1.149622$
$\odot .723578$
$-2.073963$
$-1.315012$
$-0.371520$
0.052065
1.207548
2.168403
$-1.408092$
1.437149
$-0.389633$
1.364500
1.204517
$-0.508300$
$\begin{array}{ll}\text { Reference energy: } & -302.8160951566 \\ \text { Total energy: } & -303.7432252337\end{array}$
$\begin{array}{ll}\text { Reference energy: } & -302.8160951566 \\ \text { Total energy: } & -303.7432252337\end{array}$

DBH transition structure: cleave 2nd CN TS(C-II), envelope $b$, endo1 $N=N$

UB3LYP/6-31G(d)

$E(U B+H F-L Y P)=-304.729991456$

Sum of electronic and zero-point Energies= $\quad-304.608943$

Sum of electronic and thermal Energies=

$-304.601713$

Sum of electronic and thermal Enthalpies=

Sum of electronic and thermal Free Energies=

-304 . 600769

-304 . 640488

$$
\begin{gathered}
\text { E (Thermal) } \\
\text { KCAL/MOL }
\end{gathered}
$$

TOTAL
CV

CAL/MOL-KELVIN 25.214
$S$

CAL/MOL-KELVIN 83.597 


$\begin{array}{rrrrr}1 & 7 & -2.480327 & -.214413 & -0.587953 \\ 2 & 7 & -2.010840 & -0.383503 & 0.270645 \\ 3 & 6 & -0.264055 & -0.105155 & 0.657300 \\ 4 & 6 & 0.523803 & -1.210268 & -0.042163 \\ 5 & 6 & 1.914208 & -0.554244 & -0.269234 \\ 6 & 6 & 1.643439 & 0.916413 & -0.089527 \\ 7 & 6 & 0.191640 & 1.212011 & 0.048387 \\ 8 & 1 & 2.634723 & -0.915621 & 0.478790 \\ 9 & 1 & 2.349986 & -0.804267 & -1.250528 \\ 10 & 1 & 2.426208 & 1.637122 & 0.125606 \\ 11 & 1 & -0.025066 & 2.087800 & 0.669367 \\ 12 & 1 & -0.343090 & 1.360882 & -0.914492 \\ 13 & 1 & -0.281490 & -0.150677 & 1.745159 \\ 14 & 1 & 0.574049 & -2.134003 & 0.540202 \\ 15 & 1 & 0.048643 & -1.450143 & -1.001533\end{array}$

DBH transition structure: cleave 2nd CN TS(C-II), envelope $b$, endo2 $N=N$

UB3LYP/6-31G(d)

$E(U B+H F-L Y P)=-304.727681780$

Sum of electronic and zero-point Energies= $\quad-304.607141$

Sum of electronic and thermal Energies= $\quad-304.599653$

Sum of electronic and thermal Enthalpies= $\quad-304.598709$

Sum of electronic and thermal Free Energies= $\quad-304.639811$

$\begin{array}{rrrrr}1 & 6 & 0.505509 & 1.300748 & -0.030278 \\ 2 & 6 & -0.290507 & 0.179213 & 0.639825 \\ 3 & 6 & 0.272216 & -1.146402 & 0.157303 \\ 4 & 1 & -0.423327 & 0.284021 & 1.715633 \\ 5 & 1 & -0.228753 & -1.434298 & -0.776443 \\ 6 & 6 & 1.773167 & -0.847646 & -0.107741 \\ 7 & 1 & 0.114425 & -1.959180 & 0.872052 \\ 8 & 1 & 2.169871 & -1.417240 & -0.961736 \\ 9 & 6 & 1.820543 & 0.637186 & -0.314463 \\ 10 & 1 & 0.592437 & 2.194429 & 0.602823 \\ 11 & 1 & 2.384102 & -1.150615 & 0.759828 \\ 12 & 7 & -2.015474 & 0.396285 & 0.121053 \\ 13 & 7 & -2.529836 & -0.406900 & -0.515954 \\ 14 & 1 & -0.002956 & 1.636477 & -0.953143 \\ 15 & 1 & 2.725801 & 1.182114 & -0.562575\end{array}$




\section{dinitrogen molecule}

B3LYP/6-31G(d)

$E(R B+H F-L Y P)=-109.524129072$

Sum of electronic and zero-point Energies= $\quad-109.518530$

Sum of electronic and thermal Energies= $\quad-109.516170$

Sum of electronic and thermal Enthalpies= $\quad-109.515225$

Sum of electronic and thermal Free Energies= $\quad-109.536980$

KCAL/MOL

TOTAL $\quad 4.995$
CV CAL/MOL-KELVIN 4.970
$S$

CAL/MOL - KELVIN
1
7
$\odot .000000$
0.000000
0.552751
2
$\odot .000000$
0.000000
$-0.552751$

B3LYP/6-311G(d,p)

$E(R B+H F-L Y P)=-109.555930156$

Sum of electronic and zero-point Energies= $\quad-109.550354$

Sum of electronic and thermal Energies= $\quad-109.547994$

Sum of electronic and thermal Enthalpies= $\quad-109.547050$

Sum of electronic and thermal Free Energies= $\quad-109.568787$

$$
\text { E (Thermal) }
$$$$
\mathrm{KCAL} / \mathrm{MOL}
$$

TOTAL

4.980
CV

CAL/MOL - KELVIN

4.970
$S$

CAL/MOL-KELVIN
1
7
$\odot .000000$
0.000000
0.547721
2
7
$\odot .000000$
0.000000
$-\odot .547721$
CASPT2 $(4,4) / 6-31 G(d) / / B 3 L Y P / 6-311 G(d, p)$
Reference energy: $\quad-109.0339486305$
Total energy: -109.2417612518
CASPT2 $(8,6) / 6-31 G(d) / / B 3 L Y P / 6-311 G(d, p)$
Reference energy: $\quad-109.0438189746$
Total energy: -109.2451494365

\begin{tabular}{|c|c|c|c|c|c|c|}
\hline \multicolumn{7}{|c|}{$\operatorname{CASSCF}(2,2) / 6-31 G *$} \\
\hline$E=$ & -108.9746958 & 715 & & & & \\
\hline Sum 0 & of electronic & and zero-point & pergies= & & -108 & 968707 \\
\hline Sum 0 & of electronic & and thermal En & gies $=$ & & -108 & 966346 \\
\hline Sum 0 & of electronic & and thermal En & alpies= & & -108 & 965402 \\
\hline Sum 0 & of electronic & and thermal $\mathrm{Fr}$ & Energies= & & -108 & 987128 \\
\hline & & $\begin{array}{c}\text { E (Thermal) } \\
\text { KCAL/MOL }\end{array}$ & $\mathrm{CAL} / \mathrm{MOL}$ & LVIN & & $\begin{array}{c}\mathrm{S} \\
/ M O L-K E L V I N\end{array}$ \\
\hline TOTAL & & 5.239 & & & & 45.725 \\
\hline 1 & 7 & & $0.00000 \odot$ & & 0000 & 0.544357 \\
\hline 2 & 7 & & $\odot . \odot \odot \odot \odot \odot \odot$ & $\odot . \odot \odot$ & ๑๑९ & -0.544357 \\
\hline
\end{tabular}




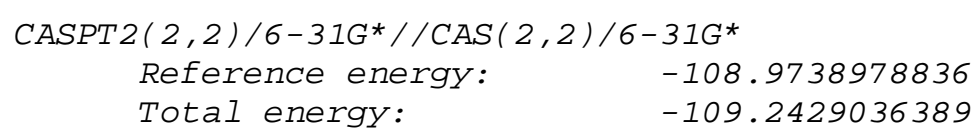

E (Thermal) $\mathrm{KCAL} / \mathrm{MOL}$

5.043

CV

$S$

TOTAL

CAL/MOL-KELVIN

CAL/MOL - KELVIN

4.970

45.775$$
7
$$
7
7
0.000000
$\odot .000000$
1
2
$0.000000 \quad 0.551216$
$0.000000 \quad-0.551216$

CASPT2 $(4,4) / 6-31 G * / / \operatorname{CAS}(4,4) / 6-31 G *$

Reference energy:

Total energy:

$-109.0340791876$

$-109.2425282034$

\author{
$\operatorname{CASSCF}(8,6) / 6-31 G$ * \\ $\mathrm{E}=-109.0448337199$ \\ Sum of electronic and zero-point Energies= $\quad-109.039154$ \\ Sum of electronic and thermal Energies= $\quad-109.036794$ \\ Sum of electronic and thermal Enthalpies= $\quad-109.035849$ \\ Sum of electronic and thermal Free Energies= $\quad-109.057599$
E (Thermal) $\mathrm{KCAL} / \mathrm{MOL}$
CV
TOTAL
5.045
CAL/MOL-KELVIN
$S$
4.970
CAL/MOL - KELVIN
45.777
1
2
$\begin{array}{ll}1 & 7 \\ 2 & 7\end{array}$
0.000000
$\odot .000000$
$\odot .000000$
$\odot .000000$
$\odot .551457$ \\ $\operatorname{CASPT} 2(8,6) / 6-31 G * / / \operatorname{CAS}(8,6) / 6-31 G^{*}$ \\ Reference energy: $\quad-109.0439709796$ \\ Total energy: \\ $-109.2459711132$
}


Raw Data from Figure 5. Change in the energy (ZPE exclusive) of the cyclohexyl-diazenyl diradical as the C4-N3 bond distance is increased from $1.56 \AA$ to $1.92 \AA$. Energies are given in a.u. Basis set: $6-31 \mathrm{G}^{*}$

(NOTE: scan was taken in two parts, which is why the $\mathrm{C}-\mathrm{N}$ distance decreases from $1.800 \AA$ to $1.560 \AA$, and then increases from $1.812 \AA$ to $1.920 \AA$ )

\begin{tabular}{lllll} 
C4-N3 & CAS $(4,4)$ & CAS $(6,6)$ & CAS $(10,8)$ & UB3LYP \\
\hline 1.800 & -341.853042 & -341.907700 & -341.921246 & -344.044348 \\
1.788 & -341.852761 & -341.907058 & -341.920710 & -344.044346 \\
1.776 & -341.852551 & -341.906483 & -341.920235 & -344.044370 \\
1.764 & -341.852409 & -341.905975 & -341.919821 & -344.044422 \\
1.752 & -341.852329 & -341.905530 & -341.919466 & -344.044502 \\
1.740 & -341.852308 & -341.905146 & -341.919167 & -344.044612 \\
1.728 & -341.852340 & -341.904817 & -341.918921 & -344.044747 \\
1.716 & -341.852419 & -341.904541 & -341.918723 & -344.044904 \\
1.704 & -341.852539 & -341.904310 & -341.918568 & -344.045081 \\
1.692 & -341.852694 & -341.904119 & -341.918451 & -344.045273 \\
1.680 & -341.852876 & -341.903961 & -341.918365 & -344.045478 \\
1.668 & -341.853079 & -341.903831 & -341.918305 & -344.045690 \\
1.656 & -341.853295 & -341.903722 & -341.918264 & -344.045907 \\
1.644 & -341.853519 & -341.903625 & -341.918234 & -344.046123 \\
1.632 & -341.853741 & -341.903536 & -341.918210 & -344.046335 \\
1.620 & -341.853955 & -341.903445 & -341.918183 & -344.046527 \\
1.608 & -341.854154 & -341.903345 & -341.918147 & -344.046715 \\
1.596 & -341.854329 & -341.903230 & -341.918093 & -344.046885 \\
1.584 & -341.854473 & -341.903091 & -341.918013 & -344.047029 \\
1.572 & -341.854568 & -341.902920 & -341.917892 & -344.047144 \\
1.560 & -341.854626 & -341.902699 & -341.917738 & -344.047222 \\
1.812 & -341.853393 & -341.908410 & -341.921849 & -344.044374 \\
1.824 & -341.853824 & -341.909188 & -341.922512 & -344.044431 \\
1.836 & -341.854328 & -341.910030 & -341.923236 & -344.044517 \\
1.848 & -341.854905 & -341.910936 & -341.924019 & -344.044634 \\
1.860 & -341.855555 & -341.911900 & -341.924857 & -344.044780 \\
1.872 & -341.856275 & -341.912919 & -341.925747 & -344.044954 \\
1.884 & -341.857060 & -341.913987 & -341.926685 & -344.045156 \\
1.896 & -341.857906 & -341.915100 & -341.927667 & -344.045384 \\
1.908 & -341.858808 & -341.916250 & -341.928687 & -344.045637 \\
1.920 & -341.859761 & -341.917433 & -341.929742 & -344.045912
\end{tabular}

C4-N3 CASPT2 $(4,4)$ CASPT2 $(6,6)$ CASPT2 $(10,8)$ CASPT2 $(6,6) / / U D F T$

$1.560-342.914925-342.911615-342.914738-342.913137$

$1.740-342.910695-342.907114-342.910575-342.908931$

$1.800-342.909601-342.906101-342.909678-342.908261$

$1.860-342.909281-342.906000-342.909833-342.908432$

$\begin{array}{llllll}1.920 & -342.909980 & -342.907096 & -342.911010 & -342.909621\end{array}$ 
Raw Data from Figure 15. Change in the energy (ZPE exclusive) of the methyldiazenyl radical as the $\mathrm{C}-\mathrm{N}$ bond distance is increased from $1.50 \AA$ to $1.92 \AA$. Energies are given in a.u. Basis set 6-31G* (CAS and CASPT2), 6-311G** (UB3LYP).

C $-\mathrm{N} \quad \operatorname{CAS}(3,3) \quad \mathrm{CAS}(5,5) \quad \operatorname{CAS}(9,7) \quad$ UB3LYP

$1.920-148.476626-148.534431-148.547289-149.384720$

$1.908-148.475677-148.533265-148.546263-149.384472$

$1.896-148.474772-148.532121-148.545265-149.384251$

$1.884-148.473918-148.531011-148.544301-149.384060$

$1.872-148.473123-148.529942-148.543377-149.383899$

$1.860-148.472392-148.528919-148.542498-149.383771$

$1.848-148.471730-148.527949-148.541669-149.383676$

$1.836-148.471141-148.527037-148.540894-149.383615$

$\begin{array}{llllll}1.824 & -148.470627 & -148.526187 & -148.540175 & -149.383589\end{array}$

$1.812-148.470192-148.525403-148.539516-149.383598$

$\begin{array}{llllll}1.800 & -148.469836 & -148.524688 & -148.538920 & -149.383642\end{array}$

$1.788-148.469559-148.524044-148.538389-149.383721$

$1.776-148.469360-148.523473-148.537922-149.383835$

$1.764-148.469236-148.522974-148.537520 \quad-149.383982$

$1.752-148.469184-148.522546-148.537182-149.384162$

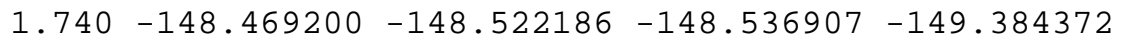

$1.728-148.469278-148.521891-148.536690-149.384610$

$1.716-148.469414-148.521656-148.536529-149.384874$

$1.704-148.469601-148.521478-148.536419-149.385162$

$1.692-148.469832-148.521349-148.536354-149.385469$

$1.680-148.470099-148.521263-148.536330-149.385794$

$\begin{array}{llllll}1.668 & -148.470397 & -148.521215 & -148.536340 & -149.386131\end{array}$

$1.656-148.470716-148.521195-148.536376-149.386476$

$1.644-148.471044-148.521198-148.536433-149.386826$

$1.632-148.471390-148.521215-148.536501-149.387176$

$1.620-148.471728-148.521239-148.536576-149.387520$

$1.608-148.472056-148.521260-148.536646-149.387853$

$1.596-148.472366-148.521272-148.536708-149.388151$

$1.584-148.472649-148.521262-148.536748-149.388445$

$1.572-148.472896-148.521232-148.536760-149.388711$

$1.560-148.473099-148.521163-148.536735-149.388942$

$1.548-148.473249-148.521048-148.536664-149.389132$

$1.536-148.473337-148.520879-148.536537-149.389272$

$1.524-148.473353-148.520647-148.536345-149.389355$

$1.512-148.473288-148.520341-148.536078-149.389374$

$1.500-148.473131-148.519953-148.535725-149.389334$

C-N CASPT2 $(3,3)$

$1.512-148.894017$

$1.524-148.893942$

$1.740-148.886369$

$1.800-148.884627$

$1.860-148.883921$

$1.920-148.884433$

C-N CASPT $(9,7)$

$1.512-148.894480$

$1.680-148.889200$

$1.800-148.885587$

$1.860-148.885384$

$1.920-148.886484$
C-N CASPT2 $(5,5)$

$1.512-148.890961$

$1.596-148.888919$

$1.656-148.886367$

$1.740-148.882865$

$1.800-148.881225$

$1.860-148.880849$

$1.920-148.881886$

C-N CASPT2 $(5,5) / /$ UDFT

$1.512-148.891105$

$1.740-148.883154$

$1.800-148.881725$

$1.860-148.881498$

$1.920-148.882590$ 
Raw Data from Figure 16. Change in the enthalpy, free energy, and T $\Delta S$ of

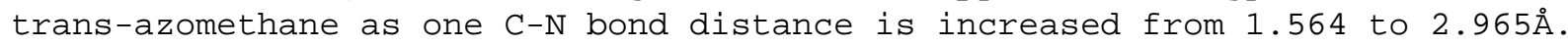
The thermodynamic correction factors were calculated at $565 \mathrm{~K}$ and $1 \mathrm{~atm}$. Basis set: 6-311G* (UB3LYP) and 6-31G* (CASPT2).

\begin{tabular}{lllll} 
C-N & UB3LYP(a.u. ) & H corr & G corr & S \\
\hline 1.465 & -189.328011 & 0.100247 & 0.024110 & 84.56 (fully optimized) \\
1.590 & -189.322627 & 0.099713 & 0.022597 & 85.65 \\
1.715 & -189.310922 & 0.099218 & 0.020394 & 87.55 \\
1.840 & -189.297142 & 0.096996 & 0.019416 & 86.16 \\
1.965 & -189.283412 & 0.096666 & 0.017978 & 87.39 \\
2.090 & -189.270687 & 0.096366 & 0.016524 & 88.68 \\
2.215 & -189.259343 & 0.095971 & 0.014653 & 90.32 \\
2.340 & -189.252058 & 0.095481 & 0.011694 & 93.06 \\
2.465 & -189.248239 & 0.095148 & $9.017000 \mathrm{e}-3$ & 95.66 \\
2.590 & -189.246242 & 0.094924 & $6.685000 \mathrm{e}-3$ & 98.00 \\
2.715 & -189.245184 & 0.094780 & $4.617000 \mathrm{e}-3100.14$ \\
2.840 & -189.244598 & 0.094693 & $2.744000 \mathrm{e}-3$ & 102.12 \\
2.965 & -189.244246 & 0.094644 & $1.040000 \mathrm{e}-3$ & 103.96
\end{tabular}

C-N CASPT2 $(6,6) / /$ UB3LYP

$2.34-188.578137$

$2.47-188.573586$

$2.59-188.570627$

$2.72-188.568723$

$2.84-188.567481$ 
Raw Data from Figure 19. Change in the energy (ZPE exclusive) of the cyclopentyl-diazenyl diradical as the $\mathrm{C}-\mathrm{N}$ bond distance is increased from $1.56 \AA$ to $1.92 \AA$. Energies are given in a.u. Basis set $6-31 \mathrm{G}^{*}$.

(NOTE: CAS scans were taken in two parts.)

\begin{tabular}{llll}
$\mathrm{C}-\mathrm{N}$ & $\mathrm{CAS}(4,4)$ & $\mathrm{CAS}(6,6)$ & $\mathrm{CAS}(10,8)$ \\
\hline 1.800 & -302.818498 & -302.873012 & -302.886699 \\
1.788 & -302.818283 & -302.872435 & -302.886225 \\
1.776 & -302.818135 & -302.871925 & -302.885811 \\
1.764 & -302.818054 & -302.871481 & -302.885458 \\
1.752 & -302.818034 & -302.871099 & -302.885163 \\
1.740 & -302.818071 & -302.870777 & -302.884923 \\
1.728 & -302.818159 & -302.870511 & -302.884735 \\
1.716 & -302.818292 & -302.870294 & -302.884594 \\
1.704 & -302.818464 & -302.870121 & -302.884495 \\
1.692 & -302.818669 & -302.869987 & -302.884432 \\
1.680 & -302.818900 & -302.869885 & -302.884399 \\
1.668 & -302.819150 & -302.869809 & -302.884390 \\
1.656 & -302.819413 & -302.869753 & -302.884399 \\
1.644 & -302.819680 & -302.869708 & -302.884419 \\
1.632 & -302.819946 & -302.869668 & -302.884443 \\
1.620 & -302.820203 & -302.869627 & -302.884463 \\
1.608 & -302.820444 & -302.869576 & -302.884472 \\
1.596 & -302.820660 & -302.869508 & -302.884463 \\
1.584 & -302.820845 & -302.869415 & -302.884428 \\
1.572 & -302.820991 & -302.869291 & -302.884359 \\
1.560 & -302.821089 & -302.869125 & -302.884248 \\
& & & \\
1.800 & -302.818498 & -302.873012 & -302.886699 \\
1.812 & -302.818787 & -302.873659 & -302.887242 \\
1.824 & -302.819152 & -302.874372 & -302.887846 \\
1.836 & -302.819591 & -302.875154 & -302.888510 \\
1.848 & -302.820103 & -302.875999 & -302.889233 \\
1.860 & -302.820689 & -302.876904 & -302.890014 \\
1.872 & -302.821346 & -302.877866 & -302.890848 \\
1.884 & -302.822070 & -302.878879 & -302.891731 \\
1.896 & -302.822857 & -302.879939 & -302.892660 \\
1.908 & -302.823701 & -302.881039 & -302.893629 \\
1.920 & -302.824599 & -302.882175 & -302.894634
\end{tabular}

$\mathrm{C}-\mathrm{N} \quad$ UB3LYP

$1.560-304.733703$

$1.572-304.733571$

$1.584-304.733409$

$1.596-304.733217$

$1.608-304.733000$

$1.620-304.732764$

$1.632-304.732514$

$1.644-304.732255$

$1.656-304.732001$

$1.668-304.731738$

$1.680-304.731478$

$1.692-304.731226$

$1.704-304.730984$

$1.716-304.730758$

$1.728-304.730548$

$1.740-304.730359$

$1.752-304.730192$

$1.764-304.730049$

$1.776-304.729933$

$1.788-304.729844$

$1.800-304.729784$

$1.812-304.729753$

$1.824-304.729753$

$1.836-304.729784$

$1.848-304.729845$

$1.860-304.731334$

$1.872-304.731495$

$1.884-304.731681$

$1.896-304.731890$

$1.908-304.732121$

$1.920-304.732373$

C-N CASPT2 $(4,4)$ CASPT2 $(6,6)$ CASPT2 $(10,8) \quad$ CASPT2//UDFT

\begin{tabular}{llllll}
\hline 1.560 & -303.748612 & -303.745150 & -303.748379 & -303.746442
\end{tabular}

$1.740-303.743095-303.739393-303.742982-303.741079$

$\begin{array}{llllll}1.800 & -303.741526 & -303.737915 & -303.741649 & -303.739919\end{array}$

$1.860-303.740784-303.737358-303.741347$

$1.920-303.741289-303.738024-303.742112$ 
Raw Data from Figure 21. Change in the energy (ZPE exclusive) of the cyclopentyl-diazenyl diradical as the $\mathrm{H} 1 \mathrm{C} 2 \mathrm{C} 3 \mathrm{H} 4$ dihedral is rotated from 20 to 70 degrees. Energies are given in a.u. Basis set: 6-31G*.

\begin{tabular}{lllll} 
HCCH & UB3LYP & PT2 $(6,6) / /$ UDFT & CAS $(4,4)$ & PT2 $(4,4) / / C A S(4,4)$ \\
\hline 20.00 & -304.733033 & -303.746435 & -302.821009 & -303.748492 \\
25.00 & -304.733338 & -303.746639 & -302.821108 & -303.748703 \\
30.00 & -304.733567 & -303.746781 & -302.821130 & -303.748857 \\
35.00 & -304.733722 & -303.746851 & -302.821085 & -303.748947 \\
40.00 & -304.733804 & -303.746856 & -302.820990 & -303.749062 \\
45.00 & -304.733817 & -303.746793 & -302.820884 & -303.749209 \\
50.00 & -304.733760 & -303.746670 & -302.820831 & -303.749211 \\
55.00 & -304.733633 & -303.746496 & -302.820825 & -303.748986 \\
$60.0 \odot$ & -304.733437 & & -302.820802 & -303.748670 \\
65.00 & -304.733172 & -303.745913 & -302.820718 & -303.748286 \\
70.00 & -304.732842 & -303.745529 & -302.820551 & -303.747851
\end{tabular}

